\title{
Semi-Parametric Estimation and Inference for the Mean Outcome of the Single Time-Point Intervention in a Causally Connected Population
}

\author{
${ }^{1}$ Department of Biostatistics, University of California Berkeley, 101 Haviland Hall, Berkeley, CA 94720, USA, E-mail: \\ oleg.sofrygin@gmail.com
}

\begin{abstract}
:
We study the framework for semi-parametric estimation and statistical inference for the sample average treatment-specific mean effects in observational settings where data are collected on a single network of possibly dependent units (e.g., in the presence of interference or spillover). Despite recent advances, many of the current statistical methods rely on estimation techniques that assume a particular parametric model for the outcome, even though some of the important statistical assumptions required by these methods are often violated in observational network settings. In this work we rely on recent methodological advances in the field of targeted maximum likelihood estimation (TMLE) and describe an estimation approach that permits for more realistic classes of data-generative models while providing valid inference in the context of observational network-dependent data. We start by assuming that the true data-generating distribution belongs to a large class of semi-parametric statistical models. We then impose some restrictions on the possible set of such distributions. For example, we assume that the dependence among the observed outcomes can be fully described by an observed network. We then show that under our modeling assumptions, our estimand can be described as a functional of the mixture of the observed data-generating distribution. With this key insight in mind, we describe the TMLE for possibly-dependent units as an iid data algorithm and we demonstrate the validity of our approach with a simulation study. Finally, we extend prior work towards estimation of novel causal parameters such as the unit-specific indirect and direct treatment effects under interference and the effects of interventions that modify the structure of the network.
\end{abstract}

Keywords: networks, dependent data, semi-parametric estimation, TMLE, interference

DOI: 10.1515/jci-2016-0003

\section{Introduction}

\subsection{Motivation}

In this paper we are concerned with estimation and inference for the sample average treatment effect [1] in an observational setting that involves members of a single connected network. Valid statistical inference in such settings presents a number of significant challenges. For example, the frequently made assumption of independence among units is generally violated when data is collected on a population of connected units, since the network interactions will often cause the exposure of one unit to have an effect on the outcomes of other connected units. In general, statistical methods for estimation and inference in observational network data are faced with three key challenges that set such data apart from the classical statistical methods for independent observational data: (i) the outcome for each unit can be a function of the treatment assignments of other units that are connected to the unit through its network, an occurrence referred to as interference or spillover [2,3]; (ii) the outcome of each unit can be a function of the baseline covariates of other units that are connected to the unit through its network, sometimes referred to as network-correlated outcomes [4]; and (iii) the observed exposure allocation for each unit can be a function of the baseline covariates of other units. As a result, the sample units are not independent, and, in fact, one only observes a single draw from the true data generating distribution. Therefore, classical statistical methods that assume independence among the observed outcomes will be often overly optimistic and invalid for quantifying the variability of estimators in such data. In addition, many of the current estimation procedures for observational network data assume a particular class of parametric or 
restrictive classes of semi-parametric models for the observed data-generating distribution, which makes these methods highly susceptible to bias due to model misspecification [5, 6].

Targeted maximum likelihood (or minimum loss-based) estimation (TMLE) $[7,8]$ is a general framework for constructing asymptotically linear and efficient substitution estimators, that belong to a much larger class of semi-parametric models, while providing asymptotically valid statistical inference. Recently, the TMLE framework has been extended to estimation of treatment effects in dependent observational data [9], where the dependence among units is described by the network of connections formed by these units (e.g., social or geographical networks). Our aim will be to provide an accurate reflection of the background knowledge available for a given scientific problem, while still being able to perform valid statistical estimation. Thus, we start by assuming a realistic semi-parametric statistical model for the generating distribution of observed network data, which places minimal restrictions on the set of such possible data-generating distributions.

In this article we generalize the previously described TMLE from van der Laan [9] to allow estimation of novel causal parameters among units that might be dependent. In particular, we describe the TMLE for estimating the treatment effect under arbitrary unit-specific stochastic interventions on groups of friends, e.g., interventions that characterize the indirect and direct treatment effect under interference [10]. We also extend our framework towards estimation of parameters defined by interventions that statically or stochastically modify the initial network structure. In addition, we describe a new asymptotic variance estimator that improves upon the previously proposed estimator from van der Laan [9]. Finally, we validate our theoretical results with a simulation study that compares TMLE to two other estimators. Our simulations demonstrate that consistent estimation and valid asymptotic inference of the sample average treatment effects for a single time point stochastic interventions is possible in this larger class of semi-parametric models, even in observational network data where the dependence between units is induced by the known network structure.

\subsection{Brief review of relevant literature}

The literature on networks and causal inference in network data is rapidly evolving. However, the existing statistical methods for performing estimation and inference for causal effects in networks are limited and the literature on this subject has only recently started to develop [9-13]. Our review is not intended to be exhaustive, instead, we focus on the key aspects and challenges of statistical estimation of treatment effects in observational network data. Most of the recently proposed approaches can be categorized as relying on either the assumption of randomized exposures across units [4, 14-22], or on parametric modeling of the outcome as a particular function of the unit's network. Some of the parametric approaches applied in the network settings include generalized linear models (GLMs) and generalized estimating equations (GEEs) [5, 6], methods which have important limitations [11, 23-26]. For one, GLMs and similar modeling techniques require making strong, simplifying modeling assumptions about the underlying data generating process. Hence, model misspecification for GEEs and GLMs in the network data settings is a major cause of concern. Perhaps more importantly, performing valid statistical inference with GLMs and other similar statistical techniques generally requires independence of the observational units, an assumption that is unlikely to hold due to the very nature of the network data. It has also been previously described that application of such standard statistical procedures to dependent data will result in invalid and generally anti-conservative statistical inference [11, 23].

In addition, a few promising methodological approaches to estimation in network data have begun to emerge in recent years. For example, Aronow and Samii [16] proposed a Horvitz-Thompson estimator in a randomized study settings, defined the so-called "network exposure model" and derived the finite sample estimator of the variance. However, such methods are of limited utility in observational settings. Other proposed approaches for identification and estimation of treatment effects in networks include stochastic actor-oriented models [27], and a linear Bayesian modeling approach that can accommodate for network uncertainty [21]. Another recently proposed approach applied the semi-parametric framework of targeted maximum likelihood estimation to the observation network data settings [9], yielding valid asymptotic inference, while allowing for a much larger and realistic class of data-generative models. We apply the latter approach in the sections that follow.

\subsection{Overview of this article}

Consider a study in which we observe a sample of $N$ units where any two units might be connected via a social network. For each unit we collect baseline covariates, a binary exposure, and a one-dimensional outcome of interest. We denote the sample by the random vector $O=(W, A, Y) \sim P_{0}$, where $W=\left(W_{i}\right)_{i=1}^{N}$ is a vector of baseline covariates across all units, $A=\left(A_{i}\right)_{i=1}^{N}$ is a vector of exposures, $Y=\left(Y_{i}\right)_{i=1}^{N}$ is a vector of outcomes, and 
$P_{0}$ belongs to a large semi-parametric model. We assume each $W_{i}$ has finite support, each $A_{i}$ is binary, and $Y_{i}$ is either binary (e.g., indicating survival beyond a specific time point, or the success of a particular intervention) or bounded (e.g., a count of the number of times an event of interest has occurred during the follow-up period, or a continuous measure of a biomarker level at the end of the study). For each unit $i$ in the sample, we also collect the information on other units in $\{1, \ldots, N\} \backslash\{i\}$ that are connected to (or influence) $i$. These units are referred to as " $i$ 's friends", and this set is denoted by $F_{i} \subseteq\{1, \ldots, N\}$. It is assumed that $F_{i}$ is recorded at baseline, along with other baseline covariates, and it is assumed fixed. Additionally, we allow $\left|F_{i}\right|$, the number of friends for unit $i$, to vary in $i$, but assume that this number is bounded by some known global constant $K$ that doesn't depend on $N$. The vector $F=\left(F_{i}\right)_{i=1}^{N}$ is then referred to as the "network profile" of $O$. For example, in an experiment evaluating the effects of introducing a new service to an online social network, for each unit, $F_{i}$ could denote the set of all online friends of $i$, whose exposure status may influence $i$ 's outcome. Alternatively, in a study of the effects of early HIV treatment initiation, $F_{i}$ could be the set of all sexual partners of unit $i$. We allow for the following types of between-unit dependencies: (i) the unit-level exposures can depend on baseline data of itself and other units, and (ii) unit-level outcomes can depend on baseline and exposure data of itself and other units. An important ingredient of our modeling approach is to assume that any dependence of each unit $i$ on other units has to be fully described by the known network. Specifically, we assume that the dependence of $i^{\prime} \mathrm{s}$ treatment and outcome on other units is limited to the set of $i$ 's friends. A second important ingredient is the assumption that these dependencies can be accurately described with some known summary measures, which map the data collected on friends of each unit into a summary that has the same dimension for all units.

We now wish to estimate and perform valid inference for the sample-average of the unit-specific mean outcomes, denoted as $\psi_{0}$ and defined as $1 / N \sum_{i=1}^{N} E\left[E_{g^{*}}\left(E\left(Y_{i} \mid A, W\right) \mid W\right)\right]$, where the $N$ exposures are assigned according to some user-specified stochastic intervention $g^{*}$. That is, $g^{*}$ is a fixed conditional density for drawing exposures $A$, given baseline covariates $W$. Under additional causal assumptions, this statistical quantity will be equal to the sample average of the expected counterfactual outcomes, defined as the expected outcomes which would have been obtained if the units in the sample had actually been treated according to the treatment regime specified by $g^{*}$ [9]. We note that the definition of intervention $g^{*}$ is kept general, allowing for any static, dynamic, or stochastic single time point interventions. We also note that the definition of the statistical parameter can be extended to multiplicative or additive sample average treatment effects $[1,28]$. Additionally, this statistical parameter is defined with respect to a given network profile $F$ and given sample size $N$, and thus should be regarded as a type of a data-adaptive statistical parameter [29], since its true value is allowed to change for different sample sizes and different network structures. Finally, we note that the statistical parameter defined in this manner has a generally meaningful statistical interpretation, even when the required causal assumptions do not hold, and our focus is only on the aspects of statistical estimation of such parameters in the context of the semi-parametric modeling framework.

Having introduced the key notation, we note that the above defined parameter $\psi_{0}$ depends on the joint distribution of the observed data only as a function of the mixture of the unit-specific distributions, and we use $\bar{P}$ to denote this mixture. In particular, we show that our parameter is equal to a mapping $\bar{\Psi}$ from $\bar{P}$. One of the advantages of the mapping $\bar{\Psi}$ is that it leads us to conclude that the estimation of $\bar{\Psi}\left(\bar{P}_{0}\right)$ should be only concerned with estimation of the relevant factors of the mixture $\bar{P}$, rather than the corresponding factors of the distribution $P$. In addition, the mapping $\bar{\Psi}$ allows us to interpret $\psi_{0}$ as a G-computation formula for the mean of iid outcomes evaluated under some fixed stochastic intervention $\bar{g}^{*}$ [30]. As we will show, this representation also implies that our statistical parameter can be consistently estimated by ignoring dependence among units and treating them as if they are independent and identically distributed (iid). This observation suggests that a large class of iid-data estimators is applicable to estimation problems, such as the one we describe in this article. Based on this key insight, the dependent-data TMLE from van der Laan [9] is then presented as a typical iid-data TMLE algorithm. We also apply the new mapping $\bar{\Psi}$ for performing statistical inference, presenting a new robust asymptotic variance estimator that remains conservative even when the outcome model $E\left(Y_{i} \mid A, W\right)$ is misspecified. We conduct a simulation study to provide a proof of concept for our framework and to assess the feasibility of unbiased estimation and inference in finite sample observational network data. We also compare the performance of TMLE to other statistical procedures using a newly developed $R$ package tmlenet [31].

The rest of the article is organized as follows: We start by formally describing the observed network data, defining the statistical model, and defining the statistical parameter of interest in Section 2. Next, in Section 3, we describe an alternative representation of our statistical parameter as a mapping from the mixture distribution $\bar{P}$, where $\bar{P}$ is derived as a function of the actual observed data distribution $P$. In Section 4, we describe how a typical iid TMLE algorithm can be applied to estimation in possibly dependent data, focusing on the sampleaverage treatment effects under single time-point stochastic intervention $g^{*}$. We then proceed by describing the asymptotic normality of this TMLE in Section 5 and discuss possible approaches to estimating its asymptotic variance in Section 6. Next, we describe a simulation study that examines the finite sample performance of the proposed TMLE, and that of the new variance estimator, in Section 7. We then describe in Section 8 how 
our framework generalizes to estimation of parameters indexed by arbitrary collections of stochastic interventions or by interventions on the network structure. We conclude with a discussion of the relative merits and limitations of our proposed approach in Section 1.

\subsection{A note on notation}

Throughout this article we use the bold font capital letters, such as $O$, to denote random vectors that include observations on all $N$ units, and bold font small letters, such as $o$, to denote their corresponding fixed values. For example, $A$ will denote the vector of $N$ exposures, i.e., $A=\left(A_{1}, \ldots, A_{N}\right)$. We will also use the standard font capital letters with a subscript to denote the unit-specific observations, i.e., $A_{i}$ will denote the exposure for the unit $i$. Finally, we will use the over-bar symbol to denote mixture distributions across all $N$ units, as well as their corresponding random variables. For example, $\bar{P}_{W}$ will denote the mixture of $N$ unit-specific distributions of baseline covariates $W_{i}$, for $i=1, \ldots, N$, i.e., $\bar{P}_{W}=1 / N \sum_{i=1}^{N} P_{i, 0}$, and $\bar{W}$ will denote a random variable distributed according to $\bar{P}_{W}$. The only exception to this rule will be $\bar{Q}$, which will denote the conditional expectation of the unit-specific outcome $Y_{i}$, as well as the conditional expectation of the mixture-based outcome $\bar{Y}$, which happen to be equal under our statistical model.

\section{Statistical model and parameter}

Suppose $P_{0}^{N}$ is the true data generating distribution for $N$ observed and connected units, with $O=(W, A, Y) \sim$ $P_{0}^{N}$ denoting the random vector for these $N$ units and $O_{i}=\left(W_{i}, A_{i}, Y_{i}\right)$, for $i=1, \ldots, N$. The network profile $F$ is assumed recorded at baseline, i.e., $F \in W$. We also assume all $Y_{i}$ are bounded random variables. Let $\mathcal{M}$ denote a statistical model containing $P_{0}^{N}$. Since $O$ represents a network of possibly dependent units, we observe only a single draw from $P_{0}^{N}$, and as a result, are unable to estimate $P_{0}^{N}$ from this single observation $O$. We now proceed by making a series of statistical assumptions, which will allow us to learn the true distribution of $O$ based on this single draw. In particular, we introduce these assumptions by making restrictions on the set of possible distributions that belong $\mathcal{M}$. We will then define our statistical quantity of interest as a mapping $\Psi$ from $\mathcal{M}$ into the real line $\mathbb{R}$.

Following van der Laan [9], we make the following set of statistical assumptions for any $P_{0}^{N} \in \mathcal{M}$ :

A.1 Conditional on $F$, each $W_{i}$ depends on at most $K$ other observations in $W=\left(W_{1}, \ldots, W_{N}\right)$, i.e., if $\left(W_{j}: j \in S_{i}\right)$ is the set of all observations dependent with $W_{i}$ then $\max _{i}\left|S_{i}\right| \leq K$ and $K \operatorname{must}$ not depend on $N{ }^{1}$

A.2 $A=\left(A_{1}, \ldots, A_{N}\right)$ are independent, conditional on $W$;

A.3 $Y_{1}, \ldots, Y_{N}$ are independent, conditional on $(A, W)$.

These assumptions imply the following likelihood for $P^{N} \in \mathcal{M}$ :

$$
p^{N}(O)=\left[\prod_{i=1}^{N} p_{Y_{i} \mid A, W}\left(Y_{i} \mid A, W\right)\right]\left[\prod_{i=1}^{N} p_{A_{i} \mid W}\left(A_{i} \mid W\right)\right] p_{W}(W) .
$$

We also assume conditional independence implied from the known network structure:

A.4 Assume that conditional distributions $P\left(Y_{i} \mid \cdot\right)$ only depend on $\left(A_{j}, W_{j}: j \in F_{i}^{*}\right)$, for $F_{i}^{*}=F_{i} \cup\{i\}$, and similarly, $P\left(A_{i} \mid \cdot\right)$ depend on $\left(W_{j}: j \in F_{i}^{*}\right)$.

We now introduce the dimension reducing assumptions for these conditional distributions. Specifically:

B.1 Each $P\left(Y_{i} \mid \cdot\right)$ is a function of some known summary measures $a_{i}^{s}\left(\left(A_{j}, W_{j}\right): j \in F_{i}^{*}\right)$ and $w_{i}^{s}\left(\left(W_{j}: j \in F_{i}^{*}\right)\right)$, for $F_{i}^{*}=F_{i} \cup\{i\}$. Each $P\left(A_{i} \mid \cdot\right)$ is a function of the summary measure $w_{i}^{s}\left(\left(W_{j}: j \in F_{i}^{*}\right)\right)$, for $F_{i}^{*}=F_{i} \cup\{i\}$. We assume $w_{i}^{S}(\cdot)$ and $a_{i}^{S}(\cdot)$ are known functions that map into Euclidean set of constant (in $i$ ) dimension that does not depend on $N$, where $a_{i}^{s}$ map into some common space $A^{s}$, and $w_{i}^{s}$ map into some common space $U^{s}$.

We will use the following shorthand notation for the above summary measures:

$$
\begin{aligned}
W_{i}^{s} & =w_{i}^{s}(W)=w_{i}^{s}\left(W_{j}: j \in F_{i}^{*}\right) \in W^{s}, \\
A_{i}^{s} & =a_{i}^{s}(A, W)=a_{i}^{s}\left(\left(A_{j}, W_{j}\right): j \in F_{i}^{*}\right) \in A^{s},
\end{aligned}
$$


As an example, an investigator might be willing to assume that the outcomes $Y_{i}$ depend on $(A, W)$ only through summary measures $\left(a_{i}^{s}(A), w_{i}^{s}(W)\right)$, where $a_{i}^{s}(A)=\left(A_{i}, A_{i}^{c}\right)$ and $w_{i}^{s}(W)=\left(W_{i}, W_{i}^{c}\right)$ and $A_{i}^{c}$ is some one dimensional summary of exposures of $i^{\prime}$ s friends and $W_{i}^{c}$ is some one dimensional summary of baseline covariates of $i$ 's friends, where $A_{i}^{c}$ is the same function in $i$ and $W_{i}^{c}$ is also the same function in $i$. If one is unwilling to make such strong dimensionality reducing assumptions, one could instead assume $a_{i}^{s}(A)=\left(A_{j}: j \in F_{i}^{*}\right)$ and $w_{i}^{s}=\left(W_{j}: j \in F_{i}^{*}\right)$, without assuming a particular functional form of $a_{i}^{s}$ and $w_{i}^{s}$. By filling the empty spots in $a_{i}^{s}(\cdot)$ and $w_{i}^{s}(\cdot)$ with missing values one would assure that all summaries $\left(a_{i}^{s}(\cdot), w_{i}^{s}(\cdot)\right)$ are of constant dimension across $i$ and that the information on the number of friends of $i$ is also captured. In summary, we allow $A_{i}^{s}$ and $W_{i}^{s}$ to be arbitrary functions of the units' network, as long as their dimension is fixed, common-in- $i$, and doesn't depend on $N$. Applying these summary measures to the observed data, we obtain the following likelihood:

$$
p^{N}(O)=\left[\prod_{i=1}^{N} p\left(Y_{i} \mid A_{i}^{s}, W_{i}^{s}\right)\right]\left[\prod_{i=1}^{N} p\left(A_{i} \mid W_{i}^{s}\right)\right] p(W) .
$$

We are now ready to make the final set of restrictions on $\mathcal{M}$. Specifically:

C.1 Assume that all $Y_{i}$ are sampled from the same distribution $Q_{Y}$ with density given by $q_{Y}\left(Y_{i} \mid a^{s}, w^{s}\right)$, conditional on fixed values of the summary measures $\left(A_{i}^{s}, W_{i}^{s}\right)$, for $i=1, \ldots, N$. Similarly, assume that all $A_{i}$ are sampled from the same distribution given by density $g\left(A_{i} \mid w^{s}\right)$, conditional on some fixed value of the summary measures $W_{i}^{s}=w^{s}$, for $i=1, \ldots, N$.

We also assume (without loss of generality) that the densities $g$ and $q_{Y}$ are well-defined with respect to some dominating measure. Using the previous example of the summary measures, i.e., $W_{i}^{s}=\left(W_{i}, W_{j}: j \in F_{i}\right)$ and $A_{i}^{S}=\left(A_{i}, A_{j}: j \in F_{i}\right)$, this assumption implies that the two units $i$ and $j$ will be subject to the same conditional distributions for drawing their exposures and outcomes, if $i$ and $j$ have the same number of friends, same individual covariate and treatment values, and the same values for the covariates and treatments of their friends. This implies that its possible to learn the common-in- $i$ densities $q_{Y}$ and $g$ from a single (but growing) draw $O$ from $P_{0}^{N}$ as $N \rightarrow \infty$, resulting in a well-defined statistical estimation problem. We denote the joint density of conditional network exposures $A$ given $W$ by $g(A \mid W)$, with above assumptions implying the factorization $g(A \mid W)=\prod_{i=1}^{N} g\left(A_{i} \mid W_{i}^{s}\right)$. We denote the joint distribution of $W$ by $Q_{W}(W)$, making no additional assumptions of independence between $W=\left(W_{1}, \ldots, W_{N}\right)$ and we assume $q_{W}$ is a well-defined density for $Q_{W}$, with respect to some dominating measure. This final set of assumptions defines our statistical model $\mathcal{M}$, where $\mathcal{M}$ describes the set of all possible distributions $P^{N}$ for the observed dependent data $O$.

We now introduce the notation $P=P_{Q, G}$, for $Q \equiv\left(Q_{W}, Q_{Y}\right)$ and we assume the distributions $Q_{W}$ and $Q_{Y}$ are unspecified beyond the above modeling conditions A1, A3, A4, B1 and $\mathbf{C} 1$. We also note that observed exposure model for $G$ may be a restricted to incorporate the real-world knowledge about the true conditional treatment assignment, for example, when the common-in- $i g\left(A_{i} \mid W_{i}^{s}\right)$ is known, such as in a randomized clinical trial. This defines the statistical parametrization for the data-generating distribution of $O$ in terms of the distributions $Q$ and $G$, and the corresponding statistical model is defined as $M=\left\{P_{Q, G}: Q \in Q, G \in G\right\}$, where $Q$ and $G$ denote the parameter spaces for $Q$ and $G$, respectively. In particular, we denote $Q_{0}$ as $Q$ evaluated at $P_{0}^{N}$. Applying this newly introduced notation results in the likelihood:

$$
p^{N}(O)=\left[\prod_{i=1}^{N} q_{Y}\left(Y_{i} \mid A_{i}^{s}, W_{i}^{s}\right)\right]\left[\prod_{i=1}^{N} g\left(A_{i} \mid W_{i}^{s}\right)\right] q_{W}(W) .
$$

We define an intervention of interest by replacing the conditional distribution $G$ with a new user-supplied intervention $G^{*}$ that has a density $g^{*}$ that we assume is well-defined. Namely, $G^{*}$ is a multivariate conditional distribution that encodes how each intervened exposure, denoted as $A_{i}^{*}$, is generated conditional on $W$. We note that static or dynamic interventions on $A$ correspond with degenerate choices of $g^{*}$ (e.g., Gill and Robins [33], Robins [32, 34, 35], Yu and van der Laan [36]), while non-degenerate choices of $g^{*}$ are often referred to as stochastic interventions (e.g., van der Laan [9], Dawid et al. [37], Robins and Richardson [39], Muñoz and van der Laan [38], Zheng and van der Laan [40]). We assume that $A$ and $A^{*}$ belong to the same common space $A$ and we make no further restrictions on $G^{*}$. We also define $A_{i}^{* s}:=a_{i}^{s}\left(A^{*}\right)$, where $A_{i}^{* s}$ denotes the random variable implied by the summary measure $a_{i}^{s}(\cdot)$ mapping from an intervened exposure vector $A^{*}$, for $i=1, \ldots, N$. Finally, we define the post-intervention distribution $P_{Q, G^{*}}$ by replacing $G$ in $P_{Q, G}$ with a new user-supplied distribution $G^{*}$. We use $O^{*}=\left(W_{i}, A_{i}^{*}, Y_{i}^{*}\right)_{i=1}^{N}$ to denote the random variable generated under $P_{Q, G^{*}}$ and its likelihood is given by: 


$$
p_{Q, G^{*}}^{N}\left(O^{*}\right)=\left[\prod_{i=1}^{N} q_{Y}\left(Y_{i}^{*} \mid A_{i}^{* s}, W_{i}^{s}\right)\right] g^{*}\left(A^{*} \mid W\right) q_{W}(W) .
$$

The latter distribution $P_{Q, G^{*}}$ is referred to as the G-computation formula for the post-intervention distribution of $O$ under stochastic intervention $G^{*}[30]$ and it is a parameter of $P^{N}$.

Our target statistical quantity $\psi_{0}$ is now defined as a function of this post-intervention distribution (2). Specifically, it is given by:

$$
\psi_{0}=\Psi\left(P_{0}^{N}\right)=E_{q_{0}, \xi^{*}}\left[\frac{1}{N} \sum_{i=1}^{N} Y_{i}^{*}\right]
$$

which is an expectation of the sample-average of $N$ outcomes among dependent units $i=1, \ldots, N$, where the expectation is evaluated with respect to the post-intervention distribution $P_{Q, G^{*}}$. We view $\Psi\left(P_{0}^{N}\right)$ as a mapping from the statistical model $\mathcal{M}$ into $\mathbb{R}$, and we note that $\psi_{0}$ is defined conditionally on the observed network structure, $F$ and is also indexed by $N$. We also define $\bar{Q}\left(A_{i}^{s}, W_{i}^{s}\right)=\int_{y} y q_{Y}\left(y \mid A_{i}^{s}, W_{i}^{s}\right) d \mu(y)$ as the conditional mean evaluated under common-in- $i$ distribution $Q_{Y}$, and $\bar{Q}_{0}$ as $\bar{Q}$ evaluated at $P_{0}^{N}$. Note that our dimension reduction assumptions imply that $E_{P_{0}^{N}}\left[Y_{i} \mid A, W\right]=\bar{Q}_{0}\left(A_{i}^{s}, W_{i}^{s}\right)$. We also note that our parameter $\psi_{0}$ only depends on $P_{0}^{N}$ through $\bar{Q}_{0}$ and $Q_{W, 0}$, and with a slight abuse of notation we will interchangeably use $\Psi\left(P_{0}^{N}\right)$ and $\Psi\left(\bar{Q}_{0}, Q_{W, 0}\right)$. Thus, the parameter $\psi_{0}$ is indexed by $N, F$ and $G^{*}$ and can be written as:

$$
\psi_{0}=\frac{1}{N} \sum_{i=1}^{N} \int_{a, w} \bar{Q}_{0}\left(a_{i}^{s}(a, w), w_{i}^{s}(w)\right) g^{*}(a \mid w) q_{W, 0}(w) d \mu(a, w),
$$

with respect to some dominating measure $\mu(a, w)$.

One might also be interested in a target quantity defined as a contrast of two stochastic interventions. For example, one may define $\Psi^{G_{1}^{*}}\left(P_{0}^{N}\right)$ and $\Psi^{G_{2}^{*}}\left(P_{0}^{N}\right)$ as the above target parameter evaluated under stochastic interventions $G_{1}^{*}$ and $G_{2}^{*}$, respectively, then defining the target quantity as $\Psi_{1}^{*}, G_{2}^{*}\left(P_{0}^{N}\right)=\Psi^{G_{1}^{*}}\left(P_{0}^{N}\right)-\Psi^{G_{2}^{*}}\left(P_{0}^{N}\right)$. The average treatment effect over $N$ connected units is then a special case of $\Psi_{1}^{*}, G_{2}^{*}\left(P_{0}^{N}\right)$ for interventions $G_{1}^{*}, G_{2}^{*}$ defined as $g_{1}^{*}\left(1^{N} \mid w\right)=1$ and $g_{2}^{*}\left(0^{N} \mid w\right)=1$, for any $w \in W$. We will focus on the estimation of the statistical parameter $\psi_{0}$ defined for one particular $G^{*}$, noting that all of our results naturally generalize to contrasts or any other quantities that can be expressed as Euclidean-valued functions of a collection $\left\{\Psi^{*}\left(P_{0}^{N}\right): G^{*} \in G^{*}\right\}$, for a finite set of stochastic interventions $G^{*}$.

We note that by making additional untestable assumptions, one can interpret $\psi_{0}$ as a causal quantity that measures the sample-average of the expected counterfactual outcomes in a network of $N$ connected units under intervention $G^{*}$, as was previously shown in van der Laan [9]. However, these additional causal assumptions put no further restrictions on the above described probability distribution $P_{0}^{N}$, so that our statistical model $\mathcal{M}$ remains the same. Since $\mathcal{M}$ contains the true data distribution $P_{0}^{N}$, it follows that $\psi_{0}$ will always have a pure statistical interpretation as the feature $\Psi\left(P_{0}^{N}\right)$ of the data distribution $P_{0}^{N}$. For the estimation problem at hand, the causal model plays no further role: even when one does not believe any of the untestable causal assumptions, one might still argue that the statistical parameter $\psi_{0}$ represents an effect measure of interest controlling for all measured confounders. Finally, we note that the assumption A1 can be dropped entirely, by defining the target parameter $\psi_{0}$ conditionally on the observed baseline covariates $W$, as shown in van der Laan [9].

\section{Target parameter as a mapping applied to a mixture model}

The above defined target parameter $\Psi\left(P_{0}^{N}\right)$ can be represented as an alternative (and equal) mapping $\bar{\Psi}\left(\bar{P}_{0}\right)$, where $\bar{P}_{0}$ is defined as a mixture of $N$ unit-specific components of the joint data-generating distribution $P_{0}^{N}$. This leads us to another way of thinking about the estimation of our target parameter, suggesting that the problem of estimating $\psi_{0}$ should only be concerned with estimating the relevant components of the mixture $\bar{P}_{0}$. We first apply the summary measures $W_{i}^{s}=w_{i}^{s}(W)$ and $A_{i}^{s}=a_{i}^{s}(A, W)$ to the observed data $O$, mapping it into a dataset of $N$ dependent summary observations, denoted $O^{s}=\left(O_{1}^{s}, \ldots, O_{N}^{s}\right)$ and referred to as the "summary data". We assume each $O_{i}^{s}=\left(W_{i}^{s}, A_{i}^{s}, Y_{i}\right)$ is distributed according to $P_{i, 0^{\prime}}^{s}$, where $P_{i, 0}^{s}$ is implied by the joint distribution $P_{0}^{N}$ 
of $O$ and the $i$-specific summary measures $\left(w_{i}^{s}(\cdot), a_{i}^{s}(\cdot)\right)$. We assume that each $P_{i, 0}^{s}$ has a well-defined density $p_{i, 0}^{s}$ with respect to some dominating measure. As before, the set of all possible distributions of $O$ is given by the statistical model $\mathcal{M}=\left\{P_{Q, G}: Q \in Q, G \in G\right\}$, and for a given $P^{N} \in \mathcal{M}$, we first define its implied mixture $\bar{P}$ and its relevant factors, and we then describe the new mapping $\bar{\Psi}(\bar{P})$ in Theorem Theorem 3.1. Our next goal is to present the efficient influence curve (EIC) for this mapping $\bar{\Psi}\left(\bar{P}_{0}\right)$, which we do in two steps in Theorems Theorem 3.2 and Theorem 3.3.

\subsection{Mapping $\psi_{0}=\bar{\Psi}\left(\bar{P}_{0}\right)$ for the mixture distribution $\bar{P}_{0}$}

Let $O^{s}$ be the sigma-algebra for the union of the unit-specific supports of $O_{i}^{s}$, for $i=1, \ldots, N$. For a set $A \in$ $O^{s}$, define the mixture distribution $\bar{P}(A)$ as a finite mixture of $N$ unit-specific summary distributions $P_{i}^{s}$ with constant weight $1 / N$, i.e., $\bar{P}:=1 / N \sum_{i=1}^{N} P_{i}^{s}$. Let $\bar{O}^{s}$ denote the random variable drawn from such $\bar{P}$ and we assume that $\bar{P}$ has a well-defined density $\bar{p}:=1 / N \sum p_{i}^{s}$. Note that $\bar{p}$ can be factorized as follows:

$$
\begin{gathered}
\bar{p}\left(\bar{O}^{s}\right)=\bar{p}\left(\bar{Y}, \bar{A}^{s}, \bar{W}^{s}\right) \\
=\bar{q}_{Y}\left(\bar{Y} \mid \bar{A}^{s}, \bar{W}^{s}\right) \bar{g}\left(\bar{A}^{s} \mid \bar{W}^{s}\right) \bar{q}_{W}\left(\bar{W}^{s}\right),
\end{gathered}
$$

and we now describe in detail the above factors of $\bar{p}$.

First, let $Q_{W_{i}^{s}}$ denote the marginal distribution of the $i$-specific baseline summary measure $W_{i}^{s}$, with density $q_{W_{i}^{s}}\left(W_{i}^{s}\right)$ defined as the marginal of the joint density $p_{i}^{s}\left(Y_{i}, A_{i}^{s}, W_{i}^{s}\right)$. The distribution $\bar{Q}_{W}$ can then be defined as a finite mixture of these $i$-specific marginal distributions $Q_{W_{i}^{s}}$, and the density of $\bar{Q}_{W}$ can be defined as follows:

$$
\bar{q}_{W}\left(w^{s}\right):=\frac{1}{N} \sum_{i=1}^{N} q_{W_{i}^{s}}\left(w^{s}\right) .
$$

We let $\bar{W}^{s}$ denote a random variable drawn from the mixture distribution $\bar{Q}_{W}$, noting that $\bar{W}^{s}$ belongs to the same common space $U^{s}$ as all $w_{i}^{s}(W)$, for $i=1, \ldots, N$. Similarly, we let $H_{i}$ denote the $i$-specific joint distribution of the summaries $\left(A_{i}^{s}, W_{i}^{s}\right)$, with its density $h_{i}\left(A_{i}^{s}, W_{i}^{s}\right)$ implied by $p_{i}^{s}\left(Y_{i}, A_{i}^{s}, W_{i}^{s}\right)$. We also let $H_{i}^{*}$ denote the joint distribution of the summaries $\left(A_{i}^{* s}, W_{i}^{s}\right)$, where $A_{i}^{* s}$ is determined by the user-supplied stochastic intervention $G_{A^{*} \mid W}^{*}$ and the $i$-specific summary measure $a_{i}^{s}$, and we denote the density of $H_{i}^{*}$ as $h_{i}^{*}$. We also assume that these $i$-specific densities $h_{i}\left(a^{s}, w^{s}\right)$ and $h_{i}^{*}\left(a^{s}, w^{s}\right)$ are well-defined with respect to some common dominating measure $\mu_{a, w}$. We now define the mixture distribution $\bar{H}$ as a finite mixture of $i$-specific $H_{i}$, with its corresponding mixture density defined as $\bar{h}\left(a^{s}, w^{s}\right):=1 / N \sum_{i=1}^{N} h_{i}\left(a^{s}, w^{s}\right)$, and we let $\left(\bar{A}^{s}, \bar{W}^{s}\right)$ denote the random variables drawn jointly from $\bar{H}$. Next, we define an analogous mixture distribution $\bar{H}^{*}$ as a finite mixture of $i$-specific distributions $H_{i}^{*}$, with its mixture density given by $\bar{h}^{*}\left(a^{s}, w^{s}\right):=1 / N \sum_{i=1}^{N} h_{i}^{*}\left(a^{s}, w^{s}\right)$, and we let $\left(\bar{A}^{* s}, \bar{W}^{s}\right)$ denote the random variables drawn jointly from $\bar{H}^{*}$. Finally, we note that these mixture densities $\bar{h}$ and $\bar{h}^{*}$ can be factorized as follows:

$$
\begin{gathered}
\bar{h}\left(a^{s}, w^{s}\right)=\bar{g}\left(a^{s} \mid w^{s}\right) \bar{q}_{W}\left(w^{s}\right), \\
\bar{h}^{*}\left(a^{s}, w^{s}\right)=\bar{g}^{*}\left(a^{s} \mid w^{s}\right) \bar{q}_{W}\left(w^{s}\right),
\end{gathered}
$$

where $\bar{g}$ is a factor in the above factorization of the likelihood of $\bar{O}^{s}$, namely, $\bar{g}$ is the density for the conditional distribution of $\bar{A}^{s}$ given $\bar{W}^{s}$, denoted as $\bar{G} ; \bar{g}^{*}$ is the density for the conditional distribution of $\bar{A}^{* s}$ given $\bar{W}^{s}$, denoted as $\bar{G}^{*}$; and $\bar{q}_{W}$ is the previously defined marginal density for the mixture $\bar{Q}_{W}$. In a similar manner one can define the conditional distribution of $\bar{Y}$ given $\left(\bar{A}^{s}, \bar{W}^{s}\right)$, denoted as $\bar{Q}_{Y}$, with its density denoted as $\bar{q}_{Y}$, which completes the description of the three factors of $\bar{p}\left(\bar{O}^{s}\right)$. We also define a statistical model $\bar{M}$ as the space of all possible distributions $\left\{\bar{Q}_{Y}, \bar{G}, \bar{Q}_{W}\right\}$ and we note that each $\bar{P} \in \bar{M}$ is implied by some $P^{N} \in \mathcal{M}$. We also note that when $(W, A)$ are discrete, one can obtain the following intuitive analytic expressions for the above defined densities $q_{W_{i}^{s}}, h_{i}$ and $h_{i}^{*}$ :

$$
\begin{array}{rc}
q_{W_{i}^{s}}\left(w^{s}\right) & =\int_{w} I\left(w_{i}^{s}(w)=w^{s}\right) q_{W}(w) d \mu_{w}(w), \\
h_{i}\left(a^{s}, w^{s}\right) & =\int_{a, w} I\left(a_{i}^{s}(a, w)=a^{s}, w_{i}^{s}(w)=w^{s}\right) g(a \mid w) q_{W}(w) d \mu_{a, w}(a, w), \\
h_{i}^{*}\left(a^{s}, w^{s}\right) & =\int_{a, w} I\left(a_{i}^{s}(a, w)=a^{s}, w_{i}^{s}(w)=w^{s}\right) g^{*}(a \mid w) q_{W}(w) d \mu_{a, w}(a, w),
\end{array}
$$


where $\mu_{w}$ and $\mu_{a, w}$ are some dominating measures. The new mapping $\psi_{0}=\bar{\Psi}\left(\bar{P}_{0}\right)$ for our target parameter is now presented in the following theorem.

Theorem 3.1 Let $P^{N} \in \mathcal{M}$ and let $P_{i}^{s}$ denote the $i$-specific summary data distribution of $O_{i}^{s}=\left(W_{i}^{s}, A_{i}^{s}, Y_{i}\right)$. Let $\bar{P} \in \bar{M}$ be the above defined finite mixture of these $N$ unit-specific distributions $P_{i}^{s}$ and $\bar{M}$ is the above defined mixture model. Let $\bar{O}^{s}=\left(\bar{W}^{s}, \bar{A}^{s}, \bar{Y}\right) \sim \bar{P}$ denote one sample drawn from $\bar{P}$. The likelihood of $\bar{O}^{s}$ is given by:

$$
\bar{p}\left(\bar{O}^{s}\right)=q_{Y}\left(\bar{Y} \mid \bar{A}^{s}, \bar{W}^{s}\right) \bar{g}\left(\bar{A}^{s} \mid \bar{W}^{s}\right) \bar{q}_{W}\left(\bar{W}^{s}\right),
$$

where $\bar{g}$ and $\bar{q}_{W}$ are the previously defined factors of $\bar{p} ; q_{Y}$ is the density of $Q_{Y} \in \mathcal{M}$ previously defined in Section 2 , i.e., $q_{Y}$ is the common-in- $i$ conditional density of $Y_{i}$ given $\left(A_{i}^{s}, W_{i}^{s}\right)$. Due to the modeling assumptions on $\mathcal{M}$, $q_{Y}$ is also the conditional density of $\bar{Y}$ given $\left(\bar{A}^{s}, \bar{W}^{s}\right)$. It follows that $\Psi\left(P^{N}\right) \equiv \bar{\Psi}(\bar{P})$, where the new mapping $\bar{\Psi}(\bar{P})$ is given by:

$$
\begin{gathered}
\quad=E_{\bar{Q}_{W}}\left[E_{\bar{g}^{*}}\left[\bar{Q}\left(\bar{Q}^{* s}, \bar{W}^{s}\right) \mid \bar{W}^{s}\right]\right] \\
=\int_{w^{s} \in W^{s}, a^{s} \in \mathcal{A}^{s}} \bar{Q}\left(a^{s}, w^{s}\right) \bar{g}^{*}\left(a^{s} \mid w^{s}\right) d \bar{Q}_{W}\left(w^{s}\right) \\
=\frac{1}{N} \sum_{i=1}^{N} E_{Q_{W_{i}^{s}}}\left[E_{\bar{g}^{*}}\left[\bar{Q}\left(\bar{A}^{* s}, W_{i}^{s}\right) \mid W_{i}^{s}\right]\right] \\
=\frac{1}{N} \sum_{i=1}^{N} \int_{w_{i}^{s}, a^{s}} \bar{Q}\left(a^{s}, w_{i}^{s}\right) \bar{g}^{*}\left(a^{s} \mid w_{i}^{s}\right) d Q_{W_{i}^{s}}\left(w_{i}^{s}\right),
\end{gathered}
$$

and we let $\bar{Q}\left(a^{s}, w^{s}\right):=E_{Q_{\gamma}}\left[\bar{Y} \mid \bar{A}^{s}=a^{s}, \bar{W}^{s}=w^{s}\right]$. With the slight abuse of notation we interchangeably write $\bar{\Psi}\left(\bar{Q}, \bar{Q}_{W}, \bar{g}^{*}\right)$ and $\bar{\Psi}(\bar{P})$, to emphasize the fact that $\bar{\Psi}$ depends on $\bar{P}$ only through $\bar{Q}, \bar{Q}_{W}$ and $\bar{g}^{*}$.

Proof First, we show that that $q_{Y}$ is indeed the conditional density of $\bar{Y}$, given $\left(\bar{A}^{s}, \bar{W}^{s}\right)$ under $\bar{P}$. To see this, note:

$$
\begin{aligned}
\bar{p}\left(w^{s}, a^{s}, y^{s}\right) & =\frac{1}{N} \sum_{i} p_{i}^{s}\left(w^{s}, a^{s}, y\right) \\
= & \frac{1}{N} \sum_{i} p_{i}^{s}\left(y \mid w^{s}, a^{s}\right) h_{i}\left(w^{s}, a^{s}\right) \\
= & q_{Y}\left(y \mid w^{s}, a^{s}\right) \frac{1}{N} \sum_{i=1}^{N} h_{i}\left(w^{s}, a^{s}\right),
\end{aligned}
$$

where by the assumptions in Section 2 we note that the distribution $P\left(Y_{i} \mid W_{i}^{s}, A_{i}^{s}\right)$ is given by a common distribution $Q_{Y}$ with density $q_{Y}$, from which the above result follows as claimed. The equivalence $\Psi\left(P^{N}\right) \equiv \bar{\Psi}(\bar{P})$ then follows directly by applying the definitions of $\bar{g}^{*}, \bar{Q}_{W}$ and $\bar{Q}$.

The above theorem implies that the estimator of $\psi_{0}$ can be obtained from the estimators of $\bar{Q}_{0}$ and $\bar{Q}_{W, 0}$. It also gives us an alternative interpretation for our target parameter $\psi_{0}$. Namely, the mapping $\bar{\Psi}\left(\bar{P}_{0}\right)$ happens to be equal to the parameter given by the G-computation formula for the mean outcome $E \bar{Y}_{\bar{g}_{0}^{*}}$, under stochastic intervention $\bar{g}_{0}^{*}$ and the observed data $\left(\bar{W}^{s}, \bar{A}^{s}, \bar{Y}\right) \sim \bar{P}_{0}$. That is, we take the above defined conditional density $\bar{g}_{0}^{*}:=\bar{h}^{*}\left(G^{*}, Q_{W, 0}\right) / \bar{q}_{W}\left(Q_{W, 0}\right)$ as if this was a known user-supplied stochastic intervention on $\bar{A}^{s}$ given $\bar{W}^{s}$, treating $\bar{g}_{0}^{*}$ as fixed we then evaluate $E \bar{Y}_{\bar{g}_{0}^{*}}$ by first replacing $\bar{g}_{0}\left(\bar{A}^{s} \mid \bar{W}^{s}\right)$ factor of $\bar{p}\left(\bar{O}^{s}\right)$ with $\bar{g}_{0}^{*}$, then taking the expectation of $\bar{Y}$ under such modified post-intervention distribution $\bar{P}^{*}$. We also recognize that $\bar{g}_{0}^{*}$ will be generally unknown, since it depends on the true distribution of the data via $Q_{W, 0}$. Nonetheless, expressing the dependent-data parameter as some function of the mixture $\bar{P}_{0}$ implies that the estimation of this parameter can be accomplished by simply treating the observed dependent units as if they are independent and identically distributed (iid) (see Lemma Lemma 4.1 from Section 4 for a specific case of estimating $\bar{g}_{0}$ component of mixture $\bar{p})$. Hence, whenever we are concerned with estimating any parameter of $\bar{P}_{0}$, such as $\psi_{0}$ given above, we can ignore the dependence among units $O_{i}^{s}, i=1, \ldots, N$, immediately providing us with an iid-analogue estimator for $\psi_{0}$, and in our case we will undertake the iid targeted maximum likelihood estimation (TMLE) approach, as described in Section 4. Among a class of iid-analogue estimators for $\psi_{0}$, we can choose an estimator which would be efficient for iid data, and in our case, we will show that such an analogous efficient iid TMLE will also be semi-parametrically efficient in our dependent data model. 


\subsection{The efficient influence curve}

The efficient influence curve (EIC), frequently referred to as the efficient score or canonical gradient, is a key ingredient in semi-parametric efficient estimation, because it defines the linear approximation of any efficient and regular asymptotically linear estimator, and therefore provides an asymptotic bound for the variance of all regular asymptotically linear estimators [41]. Furthermore, as discussed in Section 5 of van der Laan [9], even for dependent data problems such as ours, the EIC still characterizes the limiting normal distribution of the MLE, thus establishing that if we want to construct an estimator that is asymptotically equivalent to the MLE, we need to study the EIC of our target parameter. Due to local asymptotic normality of the log-likelihood, as was argued in van der Vaart [42], the normal limiting distribution implied by the MLE is still the optimal limit distribution in the convolution theorem for efficient estimators. Our first result provides the EIC $\bar{D}^{N}$ for a data-adaptive parameter $\bar{\Psi}_{N}\left(\bar{Q}_{0}, \bar{Q}_{W, 0}\right):=\bar{\Psi}\left(\bar{Q}_{0}, \bar{Q}_{W, 0}, \bar{g}_{N}^{*}\right)$ indexed by fixed $\bar{g}^{*}=\bar{g}_{N}^{*}$, where $\bar{g}_{N}^{*}$ is an estimator of the true density $\bar{g}_{0}^{*}$. We will refer to $\bar{D}^{N}$ as the iid-EIC, since it is a direct analogue of the iid-data EIC for the parameter $E \bar{Y}_{\bar{g}^{*}}$. We then present the EIC $\bar{D}$ for our actual parameter of interest $\bar{\Psi}\left(\bar{Q}_{0}, \bar{Q}_{W, 0}, \bar{g}_{0}^{*}\right)$, defined with respect to the true density $\bar{g}_{0}^{*}$.

\section{EIC for data-adaptive parameter indexed by fixed stochastic intervention $\bar{g}_{N}^{*}$}

We now replace $\bar{g}_{0}^{*}$ by a fixed density $\bar{g}^{*}$, set equal to some data-dependent estimator $\bar{g}_{N}^{*}$ of $\bar{g}_{0}^{*}$, which is then treated as fixed. This allows us to define the data-adaptive target parameter $\bar{\Psi}_{N}\left(\bar{Q}_{0}, \bar{Q}_{W, 0}\right)$, indexed by such fixed $\bar{g}^{*}$, as $E_{\bar{Q}_{W, 0}}\left[E_{\bar{g}^{*}=\bar{g}_{N}^{*}}\left[\bar{Q}_{0}\left(\bar{A}^{s}, \bar{W}^{s}\right) \mid \bar{W}^{s}\right]\right]$. From iid results for parameters defined under fixed stochastic interventions, such as those described in Muñoz and van der Laan [38], it immediately follows that the efficient influence curve for parameter $\bar{\Psi}_{N}\left(\bar{P}_{0}\right)$ at $\bar{P}_{0} \in \bar{M}$ and $\bar{O}^{s} \sim \bar{P}_{0}$ is given by:

$$
\bar{D}^{I I D}\left(\bar{P}_{0}\right)\left(\bar{O}^{s}\right)=\frac{\bar{g}^{*}}{\bar{g}_{0}}\left(\bar{A}^{s} \mid \bar{W}^{s}\right)\left[\bar{Y}-\bar{Q}_{0}\left(\bar{A}^{s}, \bar{W}^{s}\right)\right]+\left[E_{\bar{g}^{*}=\bar{g}_{N}^{*}}\left[\bar{Q}_{0}\left(\bar{A}^{s}, \bar{W}^{s}\right) \mid \bar{W}^{s}\right]-\bar{\Psi}_{N}\left(\bar{Q}_{0}, \bar{Q}_{W, 0}\right)\right]
$$

In other words, we have obtained an efficient influence curve for the mean outcome of $\bar{Y}$ under stochastic intervention $\bar{g}^{*}$, for one observation $\bar{O}^{s} \sim \bar{P}_{0}$. We will thus refer to $\bar{D}^{I I D}\left(\bar{P}_{0}\right)\left(\bar{O}^{s}\right)$ as the iid-EIC, due to just described iid-data interpretation of parameter $\bar{\Psi}_{N}(\bar{P})$. However, we don't get to observe $\bar{O}^{s}$, instead, our observed data is $O^{s}=\left(O_{1}^{s}, \ldots, O_{N}^{s}\right)$, where $O_{i}^{s} \sim P_{i, 0}^{s}$. Nonetheless, it follows that the EIC for the actual dataadaptive parameter $\bar{\Psi}_{N}\left(\bar{P}_{0}\right)$ at $P_{0}^{N} \in \mathcal{M}$ and the observed data model $O^{s} \sim P_{0}^{N}$ is given by the sum of these iid-EICs, evaluated at $i$-specific observations $O_{i}^{s}$ and scaled by $1 / N$, i.e, the EIC for parameter $\bar{\Psi}_{N}\left(\bar{P}_{0}\right)$ is given by $1 / N \sum_{i=1}^{N} \bar{D}^{I I D}\left(\bar{P}_{0}\right)\left(O_{i}^{s}\right)$ and we also present this EIC in the following theorem.

Theorem 3.2 Suppose our parameter of interest is defined by the mapping $\bar{\Psi}_{N}(\bar{P})$, where $\bar{P} \in \bar{M}$ and $\bar{g}^{*}$ is fixed. The efficient influence curve $\bar{D}^{N}\left(P^{N}\right)\left(O^{s}\right)$ for $\bar{\Psi}_{N}(\bar{P})$, evaluated at $P^{N} \in \mathcal{M}$ and one observation $O^{s}$ (consisting of $N$ dependent units) is given by

$$
\begin{aligned}
& \bar{D}^{N}\left(P^{N}\right)\left(O^{s}\right)=\frac{1}{N} \sum_{i=1}^{N}\left(\left[\frac{\bar{g}^{*}}{\bar{g}}\left(A_{i}^{s} \mid W_{i}^{s}\right)\left(Y_{i}-\bar{Q}\left(A_{i}^{s}, W_{i}^{s}\right)\right)\right]+\left[E_{\bar{g}^{*}}\left[\bar{Q}\left(A_{i}^{s}, W_{i}^{s}\right) \mid W_{i}^{s}\right]-\Psi_{i}\left(\bar{Q}_{,}, \bar{Q}_{W}\right)\right]\right) \\
&= \\
& \frac{1}{N} \sum_{i=1}^{N}\left(D_{Y_{i}}(\bar{Q}, \bar{g})\left(O_{i}^{s}\right)+D_{W_{i}^{s}}\left(\bar{Q}, \bar{Q}_{W}\right)\left(W_{i}^{s}\right)\right),
\end{aligned}
$$

where

$$
\begin{gathered}
D_{W_{i}^{s}}\left(\bar{Q}, \bar{Q}_{W}\right)\left(W_{i}^{s}\right) \quad E_{\bar{g}^{*}}\left[\bar{Q}\left(A_{i}^{s}, W_{i}^{s}\right) \mid W_{i}^{s}\right]-\Psi_{i}\left(\bar{Q}, \bar{Q}_{W}\right) \\
=\int_{a^{s}} \bar{Q}\left(a^{s}, W_{i}^{s}\right) \bar{g}^{*}\left(a^{s} \mid W_{i}^{s}\right)-\int_{a^{s}, w^{s}} \bar{Q}\left(a^{s}, w^{s}\right) \bar{g}^{*}\left(a^{s} \mid w^{s}\right) d Q_{W_{i}^{s}}\left(w^{s}\right) .
\end{gathered}
$$

Proof See Appendix B of our technical report [43].

\section{EIC for parameter under true $\bar{g}^{*}\left(g^{*}, Q_{W, 0}\right)$}

We now consider our actual target parameter $\bar{\Psi}\left(\bar{P}_{0}\right):=\bar{\Psi}\left(\bar{Q}_{0}, \bar{Q}_{W, 0}, \bar{g}_{0}^{*}\right)$, obtained by replacing fixed $\bar{g}^{*}:=$ $\bar{g}_{N}^{*}$ in $\Psi_{N}\left(\bar{Q}_{0}, \bar{Q}_{W, 0}\right)$ with true density $\bar{g}_{0}^{*}:=\bar{h}\left(G^{*}, Q_{W, 0}\right) / \bar{q}_{W}\left(Q_{W, 0}\right)$. As a result, the EIC for the parameter $\bar{\Psi}\left(\bar{Q}_{0}, \bar{Q}_{W, 0}, \bar{g}_{0}^{*}\right)$ will contain an additional contributing term $D^{\bar{g}^{*}}\left(P_{0}^{N}\right)(W)$ due to $\Psi_{N}\left(\bar{Q}_{0}, \bar{Q}_{W, 0}\right)-$ 
$\Psi\left(\bar{Q}_{0}, \bar{Q}_{W, 0}, \bar{g}_{0}^{*}\right)$. This additional contribution is derived in Appendix $C$ of our technical report [43]. The final EIC for our actual parameter $\bar{\Psi}(\bar{P})$ is given by $\bar{D}\left(P^{N}\right)(O)=\bar{D}^{N}\left(P_{0}^{N}\right)\left(O^{s}\right)+\bar{D}^{\bar{s}^{*}}\left(P_{0}^{N}\right)(W)$ and is provided in Theorem Theorem 3.3 below.

Theorem 3.3 Suppose our parameter is given by the mapping $\bar{\Psi}(\bar{P})$ defined in Section 3.1. The efficient influence curve for $\bar{\Psi}(\bar{P})$ is given by:

$$
\begin{aligned}
\bar{D}\left(P^{N}\right)(O)= & \bar{D}^{N}\left(P^{N}\right)\left(O^{s}\right)+\bar{D}^{\bar{g}^{*}}\left(P^{N}\right)(W) \\
= & \frac{1}{N} \sum_{i=1}^{N}\left(\left[\frac{\bar{g}^{*}}{\bar{g}}\left(A_{i}^{s} \mid W_{i}^{s}\right)\left(Y_{i}-\bar{Q}\left(A_{i}^{s}, W_{i}^{s}\right)\right)\right]+\left[\int_{a^{s}} \bar{Q}\left(a^{s}, W_{i}^{s}\right) \bar{g}^{*}\left(a^{s} \mid W_{i}^{s}\right)-\Psi_{i}\left(\bar{Q}, \bar{Q}_{W}\right)\right]\right) \\
& \quad+\frac{1}{N} \sum_{i=1}^{N}\left[\int_{a^{s}} \bar{Q}\left(a^{s}, W_{i}^{s}\right) g_{i}^{*}\left(a^{s} \mid W\right)-\int_{a^{s}} \bar{Q}\left(a^{s}, W_{i}^{s}\right) \bar{g}^{*}\left(a^{s} \mid W_{i}^{s}\right)\right],
\end{aligned}
$$

where

$$
\begin{gathered}
g_{i}^{*}\left(a^{s} \mid W\right)=\int_{a} I\left(a_{i}^{s}(a, W)=a^{s}\right) g^{*}(a \mid W) d \mu_{a}(a), \\
\Psi_{i}\left(\bar{Q}, \bar{Q}_{W}\right) \quad=\int_{a^{s}, w^{s}} \bar{Q}\left(a^{s}, w^{s}\right) h_{i}^{*}\left(a^{s}, w^{s}\right) d \mu\left(a^{s}, w^{s}\right) \\
=\int_{a, w} \bar{Q}\left(a_{i}^{s}(a, w), w_{i}^{s}(w)\right) g^{*}(a \mid w) q_{W}(w) d \mu(a, w),
\end{gathered}
$$

and $\bar{D}\left(P^{N}\right)$ above can be further simplified to:

$$
\begin{gathered}
\bar{D}\left(P^{N}\right)(O)=\frac{1}{N} \sum_{i=1}^{N}\left(\left[\frac{\bar{g}^{*}}{\bar{g}}\left(A_{i}^{s} \mid W_{i}^{s}\right)\left(Y_{i}-\bar{Q}\left(A_{i}^{s}, W_{i}^{s}\right)\right)\right]+\left[\int_{a^{s}} \bar{Q}\left(a^{s}, W_{i}^{s}\right) g_{i}^{*}\left(a^{s} \mid W\right)-\Psi_{i}\left(\bar{Q}_{,}, \bar{Q}_{W}\right)\right]\right) \\
=\frac{1}{N} \sum_{i=1}^{N}\left(D_{Y_{i}}(\bar{Q}, \bar{g})\left(O_{i}^{s}\right)+D_{g_{i}^{*}}\left(\bar{Q}, \bar{Q}_{W}\right)(W)\right) .
\end{gathered}
$$

Proof See Appendix B of our technical report [43].

Suppose that $\bar{g}^{*} / \bar{g}$ is uniformly bounded on a set that contains $\left(W_{i}^{s}, A_{i}^{s}\right)$ with probability 1 , for all $i$. Using similar analysis to the one conducted in van der Laan [9], we can show that $D$ above is a doubly robust estimating function for parameter $\psi_{0}=\bar{\Psi}\left(\bar{P}_{0}\right)$, in the sense that,

$$
P_{0} \bar{D}\left(\bar{Q}, \bar{g}_{0}, \psi_{0}\right)=P_{0} \bar{D}\left(\bar{Q}_{0}, \bar{g}, \psi_{0}\right)=P_{0} \bar{D}\left(\bar{Q}_{0}, \bar{g}_{0}, \psi_{0}\right)=0,
$$

where $P_{0} f=\int f(o) d P_{0}^{N}(o)$ denotes the expectation of $f$ under distribution $P_{0}^{N}$, and $\bar{Q}=\left(\bar{Q}_{,}, \bar{Q}_{W}\right)$. This implies that any estimator that solves this equation is going to be consistent if: (1) $\bar{Q}_{W, N}$ is consistent for $\bar{Q}_{W, 0}$ and (2) at least one of the two estimators $\bar{Q}_{N}$ or $\bar{g}_{N}$ is consistent for $\bar{Q}_{0}$ or $\bar{g}_{0}$.

\section{The Targeted Maximum Likelihood Estimation (TMLE)}

We had found a new representation for our target parameter $\Psi\left(P_{0}^{N}\right)=\bar{\Psi}\left(\bar{Q}_{0}, \bar{Q}_{W, 0}\right)$, which shows that it depends on $P_{0}^{N}$ only as a function of $\bar{Q}_{Y}$ and $\bar{Q}_{W}$. Demonstrating that our parameter can be written as a mapping $\bar{\Psi}\left(\bar{Q}, \bar{Q}_{W}\right)$ is hence the first step in estimation of $\psi_{0}$. It implies that the estimation of $\psi_{0}$ should be only concerned with estimating the relevant factors of the mixture $\bar{P}_{0}$, which will be accomplished by following the standard Targeted Maximum Likelihood Estimation (TMLE) template. For the description of the TMLE framework in iid data with static interventions we refer to van der Laan and Rose [7], van der Laan and Rubin [8],Gruber and van der Laan [44], and for the TMLE in iid data with stochastic intervention we refer to Muñoz and van der Laan [38].

The TMLE for estimating $\psi_{0}$ will be described in terms of the estimators $\bar{Q}_{N}, \bar{g}_{N}, \bar{g}_{N}^{*}$ and $\bar{Q}_{W, N}$ of $\bar{Q}_{0}, \bar{g}_{0}$, $\bar{g}_{0}^{*}$ and $\bar{Q}_{W, 0}$, respectively. Our next step is then to create a targeted estimator $\bar{Q}_{N}^{*}$ of $\bar{Q}_{0}$ by updating the initial estimator $\bar{Q}_{N}$, defining the TMLE $\psi_{N}^{*}$ as the corresponding plug-in estimator for the mixture mapping $\bar{\Psi}\left(\bar{Q}_{N}^{*}, \bar{Q}_{W, N}\right)$. We define the targeted update $\bar{Q}_{N}^{*}$ based on the loss function for $\bar{Q}_{0}$ and the least favorable fluctuation submodel through $\bar{Q}_{0}$ in terms of $\bar{g}_{0}$ and $\bar{g}_{0}^{*}$. The model update $\bar{Q}_{N}^{*}$ is defined in a way so that its score 
generates the efficient influence curve $\bar{D}$ presented in Theorem Theorem 3.3. That is, the targeted estimator $\bar{Q}_{N}^{*}$ updates $\bar{Q}_{N}$ by: (1) using the estimated weights $\bar{g}_{N}^{*} / \bar{g}_{N},(2)$ using a parametric submodel $\left\{\bar{Q}_{N}\left(\varepsilon, \bar{g}_{N}^{*} / \bar{g}_{N}\right)\right\}$ through the initial estimator $\bar{Q}_{N}=\bar{Q}_{N}\left(0, \bar{g}_{N}^{*} / \bar{g}_{N}\right)$ at $\varepsilon=0$, where $\left\{\bar{Q}_{N}\left(\varepsilon, \bar{g}_{N}^{*} / \bar{g}_{N}\right)\right\}$ is referred to as the leastfavorable submodel, (3) fitting $\varepsilon$ with the standard parametric MLE, with $\varepsilon^{N}$ denoting this fit, and finally, (4) defining the targeted (updated) estimator as $\bar{Q}_{N}^{*}:=\bar{Q}_{N}\left(\varepsilon^{N}, \bar{g}_{N}^{*} / \bar{g}_{N}\right)$. We note that this TMLE is actually the usual iid TMLE algorithm for estimating the quantity $E Y_{\bar{g}^{*}}$ under fixed (data-adaptive) $\bar{g}^{*}$, treating observations $O_{i}^{s}$, for $i=1, \ldots, N$ as if they are iid.Finally, this TMLE $\psi_{N}^{*}$ solves the empirical score equation given by the efficient influence curve $\bar{D}$ from Theorem Theorem 3.3, implying that $\psi_{N}^{*}$ also inherits the double robustness property of this efficient influence curve.

\subsection{The estimator $\bar{Q}_{W, N}$ for $\bar{Q}_{W, 0}$}

We define an estimator $\bar{Q}_{W, N}$ of $\bar{Q}_{W, 0}$ by first defining the empirical counterpart $Q_{W, N}$ of $Q_{W, 0}$ that puts mass one on the observed $W=\left(W_{1}, \ldots, W_{N}\right)$, which then implies that the empirical distribution $Q_{W_{i}^{s}, N}$ of $Q_{W_{i}^{s}, 0}$ will put mass one on its corresponding observed $W_{i}^{s}=w_{i}^{s}(W)$, for $i=1, \ldots, N$. Hence, for each $w^{s} \in W^{s}$, the empirical counterpart $\bar{Q}_{W, N}\left(w^{s}\right)$ of $\bar{Q}_{W, 0}\left(w^{s}\right)$ may be defined as follows:

$$
\bar{Q}_{W, N}\left(w^{s}\right):=\frac{1}{N} \sum_{i=1}^{N} I\left(W_{i}^{s}=w^{s}\right)
$$

\subsection{The initial (non-targeted) estimator $\bar{Q}_{N}$ of $\bar{Q}_{0}$}

We assumed there is a common model $\bar{Q}_{0}$ across all $i$ and $Y_{i}$ are conditionally independent given $\left(A_{i}^{s}, W_{i}^{s}\right)$, for all $i$. Consequently, the estimation of a common $\bar{Q}_{N}$ can proceed by using the pooled summary data $\left(W_{i}^{s}, A_{i}^{s}, Y_{i}\right)$, $i=1, \ldots, N$, as if the sample is iid across $i$ and one can rely on the usual parametric MLE or loss-based crossvalidation for estimating $\bar{Q}_{N}$, as described in van der Laan [9]. Given that $Y_{i}$ can be continuous or discrete for some known range $Y_{i} \in[a, b]$, for $i=1, \ldots, N$, the estimation of $\bar{Q}_{0}$ can be based on the following log-likelihood loss,

$$
L(\bar{Q})\left(Y \mid A^{s}, W^{s}\right)=-\sum_{i=1}^{N} \log \left\{\bar{Q}\left(A_{i}^{s}, W_{i}^{s}\right)^{Y_{i}}\left(1-\bar{Q}\left(A_{i}^{s}, W_{i}^{s}\right)\right)^{1-Y_{i}}\right\},
$$

or the squared error loss

$$
L(\bar{Q})\left(O^{s}\right)=-\sum_{i=1}^{N}\left(Y_{i}-\bar{Q}\left(A_{i}^{s}, W_{i}^{s}\right)\right)^{2} .
$$

Thus, fitting $\bar{Q}_{N}$ for common $\bar{Q}_{0}=E\left[Y_{i} \mid A_{i}^{s}, W_{i}^{s}\right]$ amounts to using the summary data structure $\left(W_{i}^{s}, A_{i}^{s}, Y_{i}\right)$, for $i=1, \ldots, N$. In other words, we use the entire sample of $N$ observations for predicting $Y_{i}$. For example, for binary $Y_{i}, \bar{Q}_{N}$ can be estimated by fitting a single logistic regression model to all $N$ observations, with $Y_{i}$ as the outcome, $\left(W_{i}^{s}, A_{i}^{s}\right)$ as predictors, and possibly adding the number of friends, $\left|F_{i}\right|$, as an additional covariate. After fitting $\bar{Q}_{N}$, one generates a vector of unit-specific prediction values, $\left(\bar{Q}_{N}\left(A_{i}^{s}, W_{i}^{s}\right)\right)_{i=1}^{N}$, which are then used to build an updated version $\bar{Q}_{N}^{*}$ of $\bar{Q}_{N}$.

\subsection{Estimating $\bar{g}_{0}^{*}$ and $\bar{g}_{0}$}

We now describe a direct approach to estimation of $\bar{g}_{0}$ that relies on Lemma Lemma 4.1 below. This lemma states that a consistent estimator $\bar{g}_{N}$ of $\bar{g}_{0}$ can be obtained by taking a pooled sample $\left(A_{i}^{s}, W_{i}^{s}\right)$, for $i=1, \ldots, N$, and using the usual iid maximum likelihood-based estimation, as if we were fitting a common-in- $i$ conditional density for $A_{i}^{s}$ given $W_{i}^{s}$ and treating $\left(A_{i}^{s}, W_{i}^{s}\right)$ as independent observations. For example, if each component of $A_{i}^{S}$ is binary, and $\left|A_{i}^{S}\right|=k$ for all $i$, the conditional distribution for $\bar{g}_{0}$ could be factorized in terms of the product 
of $k$ binary conditional distributions. Each of these binary conditional distributions can be estimated with the usual logistic regression methods. We also refer to Section 7 for a running example that describes in detail this direct estimation approach. Suppose now that $g_{0}$ is known, as will be the case in a randomized clinical trial (RCT). We note that this aforementioned approach to estimating $\bar{g}_{0}$ can be easily adopted to incorporate the knowledge of true $g_{0}$. That is, one could proceed by first simulating a very large number of observations $\left(A_{j}^{s}, W_{j}^{s}\right)_{j=1}^{M}$ from $\left(g_{0}, Q_{W, N}\right)$, with $Q_{W, N}$ that puts mass one on the observed $W$, and then fitting the maximum likelihood-based estimator for $\bar{g}_{0}$, as if we were fitting a common model for $A_{i}^{s}$ given $W_{i}^{s}$, based on this very large sample that is treated as iid.

As discussed in the previous section, $\bar{g}_{0}^{*}:=\bar{h}^{*}\left(G^{*}, Q_{W, 0}\right) / \bar{q}_{W}\left(Q_{W, 0}\right)$ will generally be unknown and hence will also need to be estimated from the data, in particular, since $\bar{g}_{0}^{*}$ depends on the true distribution of the data via $Q_{W, 0}$. Therefore, we propose adopting the above approach for estimation of $\bar{g}_{0}$ towards estimation of $\bar{g}_{0}^{*}$ by simply replacing the known $g_{0}$ with known $g^{*}$. Finally, even when $g_{0}$ is unknown, such as in an observational study on $N$ connected units, one could obtain a better estimator of $\bar{g}_{0}$ by utilizing the conditional independence assumptions for observed exposures $A_{i}$, given $W_{i}^{s}$, for $i=1, \ldots, N$. Similar to estimation of $\bar{Q}_{N}$, this allows us to use loss-based cross-validation and machine learning methods to obtain a good approximation $g_{N}\left(a \mid w^{s}\right)$ for common-in- $i$ density $g_{0}\left(a \mid w^{S}\right)$, resulting in an estimator $g_{N}$ of the joint density $g_{0}$. We can now repeat the above described procedure for estimating $\bar{g}_{0}$ when $g_{0}$ is known, except using such data-adaptively estimated $g_{N}$ instead of $g_{0}$. In this manner, one can obtain sufficient approximations to true $\bar{g}_{0}$ and $\bar{g}_{0}^{*}$, by fully utilizing the actual model knowledge for $g_{0}$ and the actual knowledge of $g^{*}$.

The resulting model fits $\bar{g}_{N}^{*}$ and $\bar{g}_{N}$ are used toobtain $N$ predictions $\left(\bar{g}_{N}^{*}\left(A_{i}^{s} \mid W_{i}^{s}\right) / \bar{g}_{N}\left(A_{i}^{s} \mid W_{i}^{s}\right)\right)$, for $i=1, \ldots, N$. These predictions will be used as the unit-level weights for the TMLE update of the estimator $\bar{Q}_{N}$, as described in the following section.

Lemma 4.1 (Lemma 2 in van der Laan [9]). Let the density $\bar{g}_{0}$ be defined as $\bar{g}_{0}\left(\bar{a}^{s} \mid \bar{w}^{s}\right):=\bar{h}_{0}\left(\bar{a}^{s}, \bar{w}^{s}\right) / \bar{q}_{W, 0}\left(\bar{w}^{s}\right)$, where $\bar{q}_{W, 0}$ and $\bar{h}_{0}$ are as previously defined in Section 3.1. Let $\mathcal{H}^{g}$ be a set of functions that contain true $\bar{g}_{0}$, then

$$
\bar{g}_{0}=\arg \max _{\bar{g} \in H^{g}} E_{P_{0}^{N}}\left\{\frac{1}{N} \sum_{i} \log \bar{g}\left(A_{i}^{s} \mid W_{i}^{s}\right)\right\} .
$$

A consistent estimator $\bar{g}_{N}$ for $\bar{g}_{0}$ can be obtained by plugging in the empirical counterpart of $P_{0}^{N}$ above, resulting in an estimator:

$$
\bar{g}_{N}=\arg \max _{\bar{g} \in \mathcal{H}^{g}}\left\{\frac{1}{N} \sum_{i=1}^{N} \log \bar{g}\left(A_{i}^{S} \mid W_{i}^{s}\right)\right\} .
$$

That is, $\bar{g}_{N}$ is the maximum likelihood estimator of $\bar{g}_{0}$ that uses the pooled sample $\left(A_{i}^{s}, W_{i}^{s}\right)$, for $i=1, \ldots, N$, treating the dependent $N$ units as iid.

Proof See Appendix A of our technical report [43].

\subsection{TMLE algorithm for $N$ connected units}

Having defined the estimators $\bar{Q}_{N}, \bar{g}_{N}, \bar{g}_{N}^{*}$ and $\bar{Q}_{W, N}$, the TMLE $\psi_{N}^{*}$ is obtained by first constructing the model update $\bar{Q}_{N}^{*}$ for $\bar{Q}_{N}$, as described in step 1 . below, and then evaluating $\psi_{N}^{*}$ as a substitution estimator for the mapping $\Psi$, as described in step 2. below.

1. Define the following parametric submodel for $\bar{Q}_{N}$ : Logit $\bar{Q}_{N}(\varepsilon)=\varepsilon+\operatorname{Logit} \bar{Q}_{N}$ and define the following weighted log-likelihood loss function:

$$
L^{w}\left(\bar{Q}_{N}(\varepsilon)\right)\left(O^{s}\right)=-\sum_{i=1}^{N} \log \left\{\bar{Q}_{N}(\varepsilon)\left(A_{i}^{s}, W_{i}^{s}\right)^{Y_{i}}\left(1-\bar{Q}_{N}(\varepsilon)\left(A_{i}^{s}, W_{i}^{s}\right)\right)^{1-Y_{i}}\right\} \frac{\bar{g}_{N}^{*}}{\bar{g}_{N}}\left(A_{i}^{s} \mid W_{i}^{s}\right) .
$$

The model update $\bar{Q}_{N}^{*}$ is defined as $\bar{Q}_{N}\left(\varepsilon^{N}\right)=\operatorname{Expit}\left(\operatorname{Logit} \bar{Q}_{N}+\varepsilon^{N}\right)$, where $\varepsilon^{N}$ minimizes the above loss, i.e., $\varepsilon^{N}=\arg \min _{\varepsilon} L^{w}\left(\bar{Q}_{N}(\varepsilon)\right)\left(O^{s}\right)$. That is, one can fit $\varepsilon^{N}$ by simply running the intercept-only weighted logistic regression using the pooled sample of $N$ observations $\left(W_{i}^{s}, A_{i}^{s}, Y_{i}\right)$, for $i=1, \ldots, N$, with outcome $Y_{i}$, intercept $\varepsilon$, using offsets Logit $\bar{Q}_{N}\left(A_{i}^{s}, W_{i}^{s}\right)$, predicted weights $\bar{g}_{N}^{*}\left(A_{i}^{s} \mid W_{i}^{s}\right) / \bar{g}_{N}\left(A_{i}^{s} \mid W_{i}^{s}\right)$ and no covariates. The fitted intercept is the maximum likelihood fit $\varepsilon^{N}$ for $\varepsilon$, yielding the model update $\bar{Q}_{N}^{*}$, which can be evaluated for any fixed $\left(a^{s}, w^{s}\right)$, by first computing the initial model prediction $\bar{Q}_{N}\left(a^{s}, w^{s}\right)$ and then evaluating the update $\bar{Q}_{N}\left(\varepsilon^{N}\right)$. 
2. The TMLE $\psi_{N}^{*}=\bar{\Psi}_{N}\left(\bar{Q}_{N}^{*}, \bar{Q}_{W, N}\right)$ of $\psi_{0}$ is defined as the following substitution estimator:

$$
\psi_{N}^{*}=\frac{1}{N} \sum_{i=1}^{N} \int_{a^{s}} \bar{Q}_{N}^{*}\left(a^{s}, W_{i}^{s}\right) \bar{g}_{N, N P M L E}^{*}\left(a^{s} \mid W_{i}^{s}\right) d \mu\left(a^{s}\right),
$$

where $\bar{g}_{N, N P M L E}^{*}$ is a NPMLE substitution estimator for $\bar{g}_{0}^{*}:=\bar{h}^{*}\left(G^{*}, Q_{W, 0}\right) / \bar{q}_{W}\left(Q_{W, 0}\right)$, obtained by plugging in the user-defined $G^{*}$ and the empirical counterpart $Q_{W, N}$ for $Q_{W, 0}$ which puts mass one on observed $W=\left(W_{1}, \ldots, W_{N}\right)$. Hence, the estimator $\bar{g}_{N, N P M L E}^{*}$ is defined as follows:

$$
\bar{g}_{N, N P M L E}^{*}\left(a^{s} \mid w^{s}\right)=\frac{1 / N \sum_{i} h_{i}\left(G^{*}, Q_{W, N}\right)\left(a^{s}, w^{s}\right)}{1 / N \sum_{i} Q_{W_{i}^{s}, N}\left(w^{s}\right)},
$$

for each $\left(a^{s}, w^{s}\right) \in\left(A^{s}, W^{s}\right)$. The above TMLE $\psi_{N}^{*}$ might require evaluation of $\bar{g}_{N, N P M L E}^{*}$ for every possible $a^{s}(a, W)$ in the support of $A^{*}$, and hence could be computationally challenging to implement in practice, especially for non-degenerate $g^{*}$ and multivariate $\left(a_{i}^{S}: i=1, \ldots, N\right)$. However, we also note that the above TMLE $\psi_{N}^{*}$ takes on the following algebraically equivalent form:

$$
\psi_{N}^{*}=\frac{1}{N} \sum_{i=1}^{N} \int_{a} \bar{Q}_{N}^{*}\left(a_{i}^{s}(a, W), w_{i}^{s}(W)\right) g^{*}(a \mid W) d \mu(a),
$$

which does not require computing $\bar{g}_{N, N P M L E}^{*}$. For non-degenerate $g^{*}$, the latter expression for $\psi_{N}^{*}$ can be closely approximated by sampling from $g^{*}$ and performing Monte Carlo integration. That is, we propose evaluating $\psi_{N}^{*}$ by iterating the following procedure $j=1, \ldots, M$ times: (1) Sample $N$ observations $A_{j}^{*}=\left(A_{j, 1}^{*}, \ldots, A_{j, N}^{*}\right)$ from $g^{*}(a \mid W)$, conditionally on observed $W=\left(W_{1}, \ldots, W_{N}\right) ;(2)$ Apply the summary measure mappings, constructing the following summary dataset $\left(A_{j, i}^{* s}, W_{i}^{s}\right)$, for $i=1, \ldots, N$, where each $A_{j, i}^{* s}:=a_{i}^{S}\left(A_{j}^{*}, W\right)$; and (3) Evaluate the Monte Carlo approximation to $\psi_{N}^{*}$ for iteration $j$ as:

$$
\psi_{j, N}^{*}=\frac{1}{N} \sum_{i=1}^{N} \bar{Q}_{N}^{*}\left(A_{j, i^{\prime}}^{s *}, W_{i}^{s}\right) .
$$

The Monte Carlo estimate $\bar{\psi}_{N}^{*}$ of $\psi_{N}^{*}$ is then obtained by averaging $\psi_{j, N}^{*}$ across $j=1, \ldots, M$, where $M$ is chosen large enough to guarantee a small approximation error to $\psi_{N}^{*}$. Finally, we note that one could substantially reduce the computation time of this algorithm by simply re-using the summary datasets $\left(A_{j, i}^{* s}, W_{i}^{s}\right)$ that were already constructed while performing direct estimation of $\bar{g}_{0}^{*}$ from Section Section 4.3.

\section{Asymptotic normality of TMLE}

Having defined the TMLE $\psi_{N}^{*}=\bar{\Psi}\left(\bar{Q}_{N}^{*}, \bar{Q}_{W, N}\right)$ for our parameter $\bar{\Psi}\left(\bar{Q}_{0}, \bar{Q}_{W, 0}\right)$, our goal now is to conduct inference. We start with an asymptotic analysis of the process $\left(\psi_{N}^{*}-\bar{\Psi}_{N}\left(\bar{Q}_{0}, \bar{Q}_{W, 0}\right)\right)$, where $\bar{\Psi}_{N}\left(\bar{Q}_{0}, \bar{Q}_{W, 0}\right)$ is the previously defined data-adaptive target parameter indexed by fixed $\bar{g}_{N}^{*}$. We then show that our results can be easily extended to allow inference for our original parameter of interest $\bar{\Psi}\left(\bar{Q}_{0}, \bar{Q}_{W, 0}\right)$ defined with respect to the true $\bar{g}_{0}^{*}$.

As described in the Appendix D of our technical report [43], TMLE $\bar{\Psi}\left(\bar{Q}_{N}^{*}, \bar{Q}_{W, N}\right)$ solves the following empirical score equation:

$$
\frac{1}{N} \sum_{i=1}^{N} \bar{D}^{N}\left(\bar{Q}_{N}^{*}, \bar{Q}_{W, N}, \bar{g}_{N}\right)\left(O_{i}^{s}\right)=0,
$$

where $\bar{D}^{N}(\bar{Q}, \bar{g})$ is the EIC for the data-adaptive parameter $\bar{\Psi}_{N}(\bar{Q}):=E \bar{Y}_{\bar{g}^{*}=\bar{g}_{N}^{*}}$ (Theorem Theorem 3.2). Using the identity for $\bar{D}^{N}(\bar{Q}, \bar{g})$ shown in the Appendix D of [43], we have that:

$$
\bar{\Psi}_{N}(\bar{Q})-\bar{\Psi}_{N}\left(\bar{Q}_{0}\right)=-\bar{P}_{0} \bar{D}^{N}(\bar{Q}, \bar{g})+\bar{R}_{2}\left(\bar{Q}, \bar{Q}_{0}, \bar{g}, \bar{g}_{0}\right),
$$

where we use the notation $\bar{Q}=\left(\bar{Q}, \bar{Q}_{W}\right)$ and $\bar{R}_{2}$ is second order term provided in Appendix D of [43]. Since $\bar{P}_{0}$ is defined as a mixture $1 / N \sum_{i} P_{0, i}$, and combined with the fact that our TMLE solves the above efficient score equation, we obtain: 


$$
\bar{\Psi}_{N}\left(\bar{Q}_{N}^{*}\right)-\bar{\Psi}_{N}\left(\bar{Q}_{0}\right)=\frac{1}{N} \sum_{i}\left[D_{i}^{N}\left(\bar{Q}_{N}^{*}, \bar{g}_{N}\right)\left(O_{i}^{s}\right)-P_{0, i} D_{i}^{N}\left(\bar{Q}_{N}^{*} \bar{g}_{N}\right)\right]+\bar{R}_{2, N}
$$

By having fast enough rates for $\bar{g}_{N}$ and $\bar{Q}_{N}^{*}$ one can show that $R_{2, N}=o_{P}\left(N^{-1 / 2}\right)$ and by the empirical process and asymptotic equicontinuity analysis conducted in van der Laan [9], using the same conditions as in Theorem 2 of van der Laan [9], it follows that this empirical process applied to estimated $\bar{D}^{N}\left(\bar{Q}_{N}^{*}, \bar{g}_{N}\right)$ is up to $o_{P}\left(N^{-1 / 2}\right)$ equal to the empirical process for the fixed limit, where we use $\bar{D}_{0}^{N}$ to denote this limit and we have:

$$
\bar{\Psi}_{N}\left(\bar{Q}_{N}^{*}\right)-\bar{\Psi}_{N}\left(\bar{Q}_{0}\right)=\frac{1}{N} \sum_{i}\left[D_{i, 0}^{N}\left(O_{i}^{s}\right)-P_{0, i} D_{i, 0}^{N}\right]+o_{P}\left(N^{-1 / 2}\right) .
$$

We now perform a similar derivation which will allow us to conduct inference for the parameter $\bar{\Psi}\left(\bar{Q}_{0}\right)$ defined with respect to the true $\bar{g}_{0}^{*}:=\bar{h}^{*}\left(G^{*}, Q_{W, 0}\right) / \bar{q}_{W}\left(Q_{W, 0}\right)$. Specifically, we perform the following asymptotic expansion:

$$
\begin{array}{cc}
\bar{\Psi}_{N}\left(\bar{Q}_{N}^{*}\right)-\bar{\Psi}\left(\bar{Q}_{0}\right) \\
= & \bar{\Psi}_{N}\left(\bar{Q}_{N}^{*}\right)-\bar{\Psi}_{N}\left(\bar{Q}_{0}\right)+\bar{\Psi}_{N}\left(\bar{Q}_{0}\right)-\bar{\Psi}\left(\bar{Q}_{0}\right) \\
= & \frac{1}{N} \sum_{i}\left[D_{0, i}^{N}\left(O_{i}^{s}\right)-P_{0, i} D_{0}^{N}\right]+\frac{1}{N} \sum_{i}\left[D_{0, i}^{\bar{g}^{*}}\left(O_{i}^{s}\right)-P_{0, i} D_{0, i}^{\bar{g}^{*}}\right]+o_{P}\left(N^{-1 / 2}\right) \\
= & \frac{1}{N} \sum_{i}\left[D_{0, i}\left(O_{i}^{s}\right)-P_{0, i} D_{i, 0}\right]+o_{P}\left(N^{-1 / 2}\right),
\end{array}
$$

where $D_{0}^{\bar{g}^{*}}=1 / N \sum_{i} D_{0, i}^{\bar{g}^{*}}$ was defined as the additional contribution to the EIC in Theorem Theorem 3.3 and $D_{0, i}$ is the $i$-specific zero-mean component of that EIC:

$$
\begin{gathered}
D_{0, i}= \\
\quad\left[\frac{\bar{g}_{0}^{*}}{\bar{g}_{0}}\left(A_{i}^{s} \mid W_{i}^{s}\right)\left(Y_{i}-\bar{Q}_{0}\left(A_{i}^{s}, W_{i}^{s}\right)\right)\right] \\
+\left[\int_{a^{s}} \bar{Q}_{0}\left(a^{s}, W_{i}^{s}\right) g_{i}^{*}\left(a^{s} \mid W\right)-\int_{w} \int_{a^{s}} \bar{Q}_{0}\left(a^{s}, w_{i}^{s}(w)\right) g_{i}^{*}\left(a^{s} \mid w\right) d Q_{W, 0}(w)\right] .
\end{gathered}
$$

We also note that the above expansion must hold for our TMLE $\bar{\Psi}\left(\bar{Q}_{N}^{*}, \bar{Q}_{W, N}\right)$, since it solves the score equation given by:

$$
\frac{1}{N} \sum_{i=1}^{N} D_{i}^{\bar{g}^{*}}\left(\bar{Q}_{N}, \bar{g}_{N P M L E, N}^{*}\right)(W)=0 .
$$

By applying the analogue analysis to the one conducted in van der Laan [9], we can show that the above process converges to a normal limiting distribution at $\sqrt{N}$ rate, namely,

$$
\sqrt{N}\left(\bar{\Psi}_{N}\left(\bar{Q}_{N}^{*}\right)-\bar{\Psi}\left(\bar{Q}_{0}\right)\right) \Rightarrow_{d} N\left(0, \sigma_{0}^{2}\right),
$$

with $\sigma_{0}^{2}$ given by the following limit:

$$
\sigma_{0}^{2}=\lim _{N \rightarrow \infty} \frac{1}{N} \sum_{i, j} R(i, j) E\left[D_{0, i}\left(O_{i}^{s}\right) D_{0, j}\left(O_{j}^{s}\right)\right],
$$

for $(i, j) \in\{1, \ldots, N\}^{2}$ and $R(i, j)=1$ when $F_{i} \cap F_{j} \neq \varnothing$, and $R(i, j)=0$ otherwise, and we always have that $R(i, i)=1$, for all $i=1, \ldots, N$. We also refer to Theorem 2 in van der Laan [9] for the full list of assumptions required for asymptotic normality of $\sqrt{N}\left(\bar{\Psi}_{N}\left(\bar{Q}_{N}^{*}\right)-\bar{\Psi}\left(\bar{Q}_{0}\right)\right)$.

\section{Inference}

The previous section established that the TMLE $\bar{\Psi}_{N}^{*}$ is an asymptotically linear estimator for our assumed statistical model $M$ from Section 2. This result relied in part on the assumption A1 (weak dependence of $W$ ). In particular, this assumption states that each $W_{i}$ can be dependent on at most $K$ other observations in $W=\left(W_{1}, \ldots, W_{N}\right)$, 
where $K$ is fixed. We also derived the expression for the asymptotic variance of the TMLE $\bar{\Psi}_{N^{\prime}}^{*}$ given by $\sigma_{0}^{2}$. Our next goal is to conduct inference for the TMLE by estimating $\sigma_{0}^{2}$. However, we must note that while our assumed statistical model $\mathcal{M}$ allowed us to construct a consistent and asymptotically linear TMLE, the same model $\mathcal{M}$ will not allow us to estimate its variance, i.e., it results in non-identifiable $\sigma_{0}^{2}$. To clarify, our model assumed no additional knowledge about the joint distribution of $W$, beyond their weak dependence. The weak dependence assumption alone provided no apparent method for obtaining a valid joint likelihood of $W$. Thus, we are unable to perform any type of re-sampling from the joint distribution of $W$. For example, our proposed estimator $Q_{W, N}$ from Section 4 put mass one on $N$ observed $W$, which leads to an estimate of $\left(E_{g^{*}}\left[Y_{i} \mid W\right]-\Psi_{i, 0}\right)$ that is constant zero for all $i=1, \ldots, N$. As a result, the NPMLE estimate $Q_{W, N}$ doesn't allow for valid estimation of the unit-specific components of the efficient influence curve (EIC) that contribute to $\sigma_{0}^{2}$. In particular, we cannot estimate the terms $P_{0}\left\{\left(E_{g^{*}}\left[Y_{i} \mid W\right]-\Psi_{i, 0}\right) \times\left(E_{g^{*}}\left[Y_{j} \mid W\right]-\Psi_{j, 0}\right)\right\}$, for any dependent $i$ and $j$. As a consequence, asymptotic variance is non-identifiable and valid estimation of $\sigma_{0}^{2}$ will generally require making additional modeling restrictions for $Q_{W}$, beyond the previously assumed weak dependence of $W$.

However, if our statistical model results in a valid joint likelihood of $W$ and allows us to fit its joint distribution, then the variance $\sigma_{0}^{2}$ can be estimated. In this case one could employ the parametric bootstrap that re-samples $W$ from the joint fit of its distribution. To summarize, as long as we can fit the likelihood for $\mathrm{Q}_{W}$ (i.e., assume a model on $W$ which would allow us to do that) then the TMLE variance $\sigma_{0}^{2}$ can be consistently estimated. Finally, if we are unwilling to make additional modeling restrictions for $Q_{W}$ then only the upper bound estimates of $\sigma_{0}^{2}$ may be obtained. While we propose a possible ad-hoc upper bound estimate of $\sigma_{0}^{2}$ below, we leave its theoretical validation and the more detailed analysis of this topic for the future research.

In what follows, we provide some examples of specific modeling restrictions on $W$ (e.g., when assuming $W$ are iid) that allow us to consistently estimate the variance of the TMLE. We also describe an ad-hoc approach which may provide an estimate of the upper bound of such variance when not making any additional modeling restrictions for $Q_{W}$. Finally, we describe the inference for the parameter that conditions on all observed $W$ and thus doesn't require making any modeling assumptions about their joint distribution.

N.B. We note that our described TMLE is based on the EIC from Theorem Theorem 3.3. While this EIC was derived under no modeling restrictions on $Q_{W}$, our actual statistical model $\mathcal{M}$ from Section 2 had to assume that $Q_{W}$ satisfied the weak dependence assumption. As such, the EIC from Theorem Theorem 3.3 does not characterize the asymptotic efficiency bound within model $M$. That is, by assuming some structure on $W$ we are implicitly changing the corresponding efficiency bound of our statistical model. Thus, our proposed TMLE is no longer an asymptotically efficient estimator in a model that assumes the weak dependence of $W$. However, this restriction was crucial for proving the asymptotic linearity of TMLE and was thus unavoidable. We are also unaware of any current methods which would allow us to derive the EIC either for a model where only the weak dependence of $W$ is assumed or for some variation of this assumption.

Furthermore, putting even more modeling restrictions on $Q_{W}$ will further restrict the statistical model, implying that the previous TMLE will become even less efficient. However, these restrictions are only necessary to identify the variance, since the TMLE remains well-behaved in a bigger model $\mathcal{M}$. That is, these additional restrictions have to do with model-based variance estimation.

\subsection{Inference when assuming a restricted joint model for the baseline covariates}

Consider a special case where $W$ are iid. A valid approach to conducting inference is to use the empirical analogue estimator of $\sigma_{0}^{2}$ based on the plug-in estimates $D_{N, i}$ of $D_{0, i}$. Evaluating each $E\left[D_{N, i}\left(O_{i}^{s}\right) D_{N, j}\left(O_{j}^{s}\right)\right]$ with respect to its empirical counterpart then results in the following estimator of $\sigma_{0}^{2}$ :

$$
\sigma_{N}^{2}=\frac{1}{N} \sum_{i, j} R(i, j)\left[D_{N, i}\left(O_{i}^{s}\right) D_{N, j}\left(\bar{O}_{j}\right)\right]
$$

where

$$
\begin{aligned}
D_{N, i}\left(O_{i}^{s}\right) & = \\
& D_{i}\left(\bar{Q}_{N}, \bar{g}_{N}, \bar{g}_{N}^{*}, \psi_{N}^{*}\right)\left(O_{i}^{s}\right) \\
& =\frac{\bar{g}_{N}^{*}}{\bar{g}_{N}}\left(A_{i}^{s} \mid W_{i}^{s}\right)\left[Y_{i}-\bar{Q}_{N}\left(A_{i}^{s}, W_{i}^{s}\right)\right]+\left[\int_{a} \bar{Q}_{N}^{*}\left(a_{i}^{s}(a, W), w_{i}^{s}(W)\right) g^{*}(a \mid W)-\Psi_{i}\left(\bar{Q}_{N}^{*}, Q_{W, N}\right)\right]
\end{aligned}
$$

and 


$$
\Psi_{i}\left(\bar{Q}_{N}^{*}, Q_{W, N}\right)=\int_{w} \int_{a} \bar{Q}_{N}^{*}\left(a_{i}^{s}(a, w), w_{i}^{s}(w)\right) g^{*}(a \mid w) d Q_{W, N}(w) .
$$

We refer to Appendix B for results demonstrating the consistency of $\sigma_{N}^{2}$ when assuming iid $W$. Note that each $\Psi_{i}\left(\bar{Q}_{N}^{*}, Q_{W, N}\right)$, for $i=1, \ldots, N$, can be approximated with Monte-Carlo integration by sampling with replacement from $N$ iid baseline covariates and then sampling $N$ exposures according to $g^{*}$. We also note that for the case of iid baseline covariates one can derive the actual EIC for this specific model, as it was presented in Section 6.2 of van der Laan [9]. However, the implementation of its corresponding plug-in asymptotic variance estimator is computationally overwhelming and we hence propose using the above estimator $\sigma_{N}^{2}$ of $\sigma_{0}^{2}$. Furthermore, we know from the results for iid data that such EIC-based confidence intervals will generally provide correct coverage when $\bar{Q}_{N}$ and $\bar{g}_{N}$ are correctly specified, and will be conservative if only $\bar{g}_{N}$ is specified correctly. Analogous to these results we expect our estimator $\sigma_{N}^{2}$ to be conservative when the model for $\bar{Q}_{0}$ is misspecified, and we test the validity of this conjecture in a simulation study in Section 7. One can then construct a $95 \%$ confidence interval (CI) $\psi_{N}^{*} \pm 1.96 \sigma_{N} / \sqrt{N}$, which will provide correct asymptotic coverage.

An alternative approach that avoids assuming iid $W$ is to assume a statistical model that specifies a particular ordering of observations $i=1, \ldots, N$. This ordering, combined with the weak dependence of $W$, allows us to assume a particular statistical graph for the dependence among $\left(W_{1}, \ldots, W_{N}\right)$, thus defining a unique factorization of the joint likelihood of $W$. By putting additional modeling restriction on these individual likelihood factors we can obtain a single fit of the joint distribution $Q_{W}$. This will in turn allow us to re-sample $W$. Thus, we would be able to obtain an estimate of each $E\left[D_{0, i}\left(O_{i}^{s}\right) D_{0, j}\left(O_{j}^{s}\right)\right]$ by utilizing the same plug-in approach as in the above described iid case. Note that by having a consistent estimate of $E\left[D_{0, i}\left(O_{i}^{s}\right) D_{0, j}\left(O_{j}^{s}\right)\right]$ one can also apply the same techniques from Appendix B to demonstrate that the corresponding empirical analogue estimate $\sigma_{N}^{2}$ will be also consistent for $\sigma_{0}^{2}$.

\subsection{Ad-hoc upper bound for the variance}

An alternative approach that avoids putting additional modeling restriction on the joint distribution of $W$ is to consider various ad-hoc approaches of obtaining conservative estimates of the variance. As one of the examples, consider a plug-in estimate for $\sigma_{0}^{2}$ that is based on the following estimate of $D_{0, i}$ :

$$
D_{N, i}^{c}\left(O_{i}^{s}\right)=\frac{\bar{g}_{N}^{*}}{\bar{g}_{N}}\left(A_{i}^{s} \mid W_{i}^{s}\right)\left[Y_{i}-\bar{Q}_{N}\left(A_{i}^{s}, W_{i}^{s}\right)\right]+\left[\int_{a} \bar{Q}_{N}^{*}\left(a_{i}^{s}(a, W), w_{i}^{s}(W)\right) g^{*}(a \mid W)-\bar{\Psi}_{N}^{*}\right],
$$

where

$$
\bar{\Psi}_{N}^{*}=\frac{1}{N} \sum_{i=1}^{N} \Psi_{i}\left(\bar{Q}_{N}^{*}, Q_{W, N}\right)
$$

Note that the above $i$-specific estimates $D_{N, i}$ of $D_{0, i}$ are no longer guaranteed to have mean zero (i.e., they are not necessary properly centered), resulting in the following conservative estimate of the variance:

$$
\frac{1}{N} \sum_{i, j} R(i, j)\left[D_{N, i}^{c}\left(O_{i}^{s}\right) D_{N, j}^{c}\left(\bar{O}_{j}\right)\right]
$$

Our simulations suggest that this estimator can be quite accurate when the number of friends $\left|F_{i}\right|$ is relatively uniform, for $i=1, \ldots, N$, and that it becomes conservative for networks with skewed friend distributions (simulation results not shown). Finally, for more extreme cases, such as when the number of friends follows a power law distribution, the above plug-in variance estimator becomes overly conservative to the point of being noninformative. It is our conjecture that as the variability of individual $\Psi_{i}, 0$ increases, this estimator should become more and more conservative. We now leave a more detailed analysis of this estimator as a topic of future research.

\subsection{Inference when conditioning on the baseline covariates}

Admittedly, the assumption of iid baseline covariates $(W)$ might be too restrictive for many realistic network data generating scenarios. Similarly, the other suggested approaches will also require making specific modeling assumptions that are not necessarily supported by the data. We now propose an alternative approach 
for conducting inference by simply giving up on the marginal parameter of interest and performing inference conditionally on the observed baseline covariates $W$. This approach has been previously described in van der Laan [9], Section 8 and it results in a TMLE which is identical to the one we present in this paper. Moreover, this TMLE achieves asymptotic normality with an identifiable asymptotic variance. The asymptotic variance estimator is given by

$$
\sigma_{N}^{2}(W)=\frac{1}{N} \sum_{i=1}^{N}\left[D_{N, Y_{i}}^{2}\left(O_{i}^{s}\right)\right]
$$

where

$$
D_{N, Y_{i}}\left(O_{i}^{s}\right)=\frac{\bar{g}_{N}^{*}}{\bar{g}_{N}}\left(A_{i}^{s} \mid W_{i}^{s}\right)\left(Y_{i}-\bar{Q}_{N}\left(A_{i}^{s}, W_{i}^{s}\right)\right) .
$$

Note that this conditional TMLE doesn't require modeling the distribution of the baseline covariates and thus achieves the asymptotic normality under much weaker set of conditions for $W$ (see van der Laan [9], Section 8). Thus, conducting conditional inference in a model with weakly dependent baseline covariates is a powerful alternative, especially when one is willing to accept the conditional interpretation of the resulting inference.

\section{Simulation study}

We performed a network simulation study evaluating the finite sample bias and variance of the TMLE presented in Section 4. We also evaluated the finite sample coverage of the confidence intervals described in Section 6. In addition to TMLE, we also used the Inverse Probability of Treatment Weighted (IPTW) estimator and the G-computation substitution estimator, where both of these estimators are defined below. All estimation was performed in $R$ language [45] using a stand-alone package tmlenet [31]. The results are reported for networks consisting of $N=500$ and $N=1,000$ observations. The estimation was repeated by sampling 10,000 datasets. Due to computing time limitations, each unit in the network was allowed to be connected to at most two other units (at most two friends in $F_{i}$, for each $i=1, \ldots, N$ ). However, we note that since the same estimand would generally be obtained only once when using the actual observed data, one should be able to employ the tmlenet $R$ package for estimation in more realistic network datasets where observed units may have much higher degrees of connectivity. The data generating distribution used in these simulations is described in more detail in Appendix A. Briefly, we first sampled $N$ iid baseline covariates, $W=\left(W_{1}, \ldots, W_{N}\right)$. For each unit $i$, we then generated $F_{i}$ by first sampling its size, $\left|F_{i}\right|$, uniformly from $\{0,1,2\}$, followed by uniform sampling without replacement of $F_{i}$ from the population of $N-1$ units (all units, except $i$ ). The network-induced dependence among units was then simulated in the following manner. Each treatment $A_{i}$ was sampled as a Bernoulli random variable, with its probability of success depending on the baseline covariate values of units in $F_{i} \cup\{i\}$. Similarly, each outcome $Y_{i}$ was sampled as a Bernoulli random variable, with its probability of success depending on the baseline covariate values and treatments of units in $F_{i} \cup\{i\}$. The probability of success for each $A_{i}$ was defined as a logit-linear function of the 2-dimensional summary $\left(W_{i}, \sum_{j} W_{j}: j \in F_{i}\right)$, given as:

$$
P_{0}\left(A_{i}=1 \mid W_{i}^{S}\right)=\operatorname{expit}\left(\alpha_{0}+\alpha_{1} W_{i}+\alpha_{2} \sum_{j \in F_{i}} W_{j}\right)
$$

Similarly, the probability of success for each $Y_{i}$ was defined as a logit-linear function of the 4-dimensional summary $\left(W_{i}, \sum_{j} W_{j}, A_{i}, \sum_{j} A_{j}: j \in F_{i}\right)$, given as:

$$
\bar{Q}_{0}\left(A_{i}^{s}, W_{i}^{s}\right)=\operatorname{expit}\left(\beta_{0}+\beta_{1} A_{i}+\beta_{2} \sum_{j \in F_{i}} A_{j}+\beta_{1}^{\prime} W_{i}+\beta_{2}^{\prime} \sum_{j \in F_{i}} W_{j}\right)
$$

In contrast, the estimation of the common-in- $i \bar{Q}_{0}$ and the mixture density $\bar{g}_{0}$ was based on non-parametrically defined summary measures, i.e., we let $W_{i}^{s}=\left(\left|F_{i}\right|, W_{i}, W_{j}: j \in F_{i}\right)$ and $A_{i}^{s}=\left(A_{i}, A_{j}: j \in F_{i}\right)$, such that, for all $i,\left|W_{i}^{s}\right|=4$ and $\left|A_{i}^{s}\right|=3$. Whenever unit $i$ had fewer than 2 friends $\left(\left|F_{i}\right|<2\right)$, the remainders of $W_{i}^{s}$ and $A_{i}^{s}$ were filled with zeros to ensure the same summary measure dimensionality across $i$. The common-in-i model $\bar{Q}_{N}$ for $\bar{Q}_{0}$ was then estimated by fitting a logistic regression model to a pooled sample of $N$ units, using 
covariates $\left(A_{i}^{s}, W_{i}^{s}\right)$. The estimation of the conditional mixture density $\bar{g}_{0}\left(a^{s} \mid w^{s}\right)$ proceeded as follows. First, for any $\left(a^{s}, w^{s}\right) \in\left(\bar{A}^{s} \times U \bar{l}^{s}\right)$, such that $a^{S} \in\{0,1\}^{3}$ and $a^{s}=\left(a^{s}(1), a^{S}(2), a^{S}(3)\right)$, we factorized $P\left(\bar{A}^{s}=a^{s} \mid \bar{W}^{s}=w^{s}\right)$ as:

$$
\begin{aligned}
P\left(\bar{A}^{s}=a^{s} \mid \bar{W}^{s}=w^{s}\right)= & P\left(\bar{A}^{s}(1)=a^{s}(1), \bar{A}^{s}(2)=a^{s}(2), \bar{A}^{s}(3)=a^{s}(3) \mid \bar{W}^{s}=w^{s}\right) \\
= & P\left(\bar{A}^{s}(1)=a^{s}(1) \mid \bar{W}^{s}=w^{s}\right) \\
& \times P\left(\bar{A}^{s}(2)=a^{s}(2) \mid \bar{A}^{s}(1)=a^{s}(1), \bar{W}^{s}=w^{s}\right) \\
& \times P\left(\bar{A}^{s}(3)=a^{s}(3) \mid \bar{A}^{s}(1)=a^{s}(1), \bar{A}^{s}(2)=a^{s}(2), \bar{W}^{s}=w^{s}\right) .
\end{aligned}
$$

We then fit three separate logistic regression models, each estimating one of the factors in the above factorization, as if we were fitting common-in- $i$ models using an iid sample of $N$ observations $\left(A_{i}^{s}, W_{i}^{s}\right)$. That is, the first factor $P\left(\bar{A}^{s}(1)=1 \mid \bar{W}^{S}\right)$ was fit as if we were estimating a common-in-i model $P\left(A_{i}^{s}(1)=1 \mid W_{i}^{s}\right)$ for $N$ iid observations $\left(A_{i}, W_{i}^{s}\right)_{i=1}^{N}$ (note that $A_{i}^{S}(1)=A_{i}$ ). Similarly, the second factor was fit as if we were estimating a common-in- $i$ model for $P\left(A_{i}^{S}(2)=1 \mid A_{i}, W_{i}^{S}\right)$, and so on. The resulting three fits were then combined in order to obtain the estimate $\bar{g}_{N}\left(a^{s} \mid w^{s}\right)$ of $\bar{g}_{0}\left(a^{s} \mid w^{s}\right)$. We estimated $\bar{g}_{0}^{*}$ in a similar way, except that we first sampled a large dataset of observations $\left(A_{i}^{*}, W_{i}\right)$ from $g^{*}$ and $Q_{W, N}$, for $i=1, \ldots, m N$, then constructed the summary data $W_{i}^{s}=\left(W_{i}, W_{j}: j \in F_{i}\right), A_{i}^{* s}=\left(A_{i}^{*}, A_{j}^{*}: j \in F_{i}\right)$, and finally estimated $\bar{g}_{N}^{*}$ by factorizing $P\left(\bar{A}^{*}=a^{s} \mid \bar{W}^{s}=w^{s}\right)$ into three factors and fitting three logistic regressions to a pooled sample $\left(A_{i}^{*}, W_{i}\right)$ of $m N$ observations.

The stochastic intervention $g^{*}(A \mid W)$ was defined as a common-in- $i$ intervention $g_{p}^{*}$ on each $A_{i}$, which assigned $A_{i}=1$ with some constant probability $p$, i.e., $P\left(A_{i}^{*}=1\right)=p$. Our target parameter was then defined as the sample-average of $N$ outcomes under $g_{p}^{*}$, where we use $\psi_{0}\left(g_{p}^{*}\right)$ to denote the parameter's true value. In our simulations we then estimated a discrete dose response curve $\left\{\psi_{0}\left(g_{p}^{*}\right)\right\}$ for $p \in[0,0.1, \ldots, 0.9,1]$. We also truncated the observation-specific weights $\bar{g}_{N}^{*}\left(a^{s} \mid w^{s}\right) / \bar{g}_{N}\left(a^{s} \mid w^{s}\right)$ when their values exceeded $10^{5}$. Finally, the confidence intervals for the TMLE were constructed based on variance estimator $\sigma_{N}^{2}$ from Section 6. Lastly, we compared $\sigma_{N}^{2}$ with an alternative asymptotic variance estimator $\tilde{\sigma}_{N}^{2}$ presented in van der Laan [9], which requires the consistency of $\bar{Q}_{N}$ and $\bar{g}_{N}$ and is given by:

$$
\tilde{\sigma}_{N}^{2}=\frac{1}{N}\left[\sum_{i=1}^{N} \frac{\bar{g}_{N}^{*}}{\bar{g}_{N}}\left(A_{i}^{s}, W_{i}^{s}\right)\left(Y_{i}-\bar{Q}_{N}\left(A_{i}^{s}, W_{i}^{s}\right)\right)\right]^{2}+\frac{1}{N} \sum_{i_{1}, i_{2}=1}^{N} f_{W, i} f_{W, i_{2}} I\left(F_{i_{1}} \cap F_{i_{2}} \neq \emptyset\right)
$$

for

$$
f_{W, i}=\int_{a} \bar{Q}_{N}\left(a_{i}^{s}(a), w_{i}^{s}(W)\right) g_{i}^{*}(a \mid W)-\int_{w}\left[\int_{a} \bar{Q}_{N}\left(a_{i}^{s}(a), w_{i}^{s}(W)\right) g_{i}^{*}(a \mid w)\right] Q_{W, N}(w) .
$$

\subsection{IPTW (Horvitz-Thompson) estimator}

The IPTW estimator is based on the TMLE weights $\bar{g}_{0} / \bar{g}_{0}^{*}$ from Section 4.4 and is defined as the weighted average of the observed outcomes $Y_{i}$, weighted by $\bar{g}_{N}^{*} / \bar{g}_{N}$ :

$$
\psi_{I P T W, N}=\frac{1}{N} \sum_{i=1}^{N} Y_{i}\left[\frac{\bar{g}_{N}^{*}}{\bar{g}_{N}}\left(A_{i}^{s} \mid W_{i}^{s}\right)\right],
$$

where $\bar{g}_{N}$ is an estimator of the conditional mixture $\bar{g}_{0}\left(G_{0}, Q_{W, 0}\right)$ defined in Section 3.1 and $\bar{g}_{N}^{*}$ is an estimator of $\bar{g}_{0}^{*}\left(G^{*}, Q_{W, 0}\right)$, also defined in Section 3.1. The estimators $\bar{g}_{N}$ and $\bar{g}_{N}^{*}$ are described in general in Section 4 and are also described above for the case of non-parametrically defined summary measures. We also conducted inference for $\psi_{I P T W, N}$ by relying on the same ideas described in Section 6 . That is, we used the iid-data influence curve IC $\left(\bar{P}_{0}\right)$ of $\psi_{I P T W, N}$ in a model that assumes $\bar{g}_{0}^{*}$ and $\bar{g}_{0}$ are known, characterizing the asymptotic variance of $\psi_{I P T W, N}$ by the following limit:

$$
\sigma_{I P T W, 0}^{2}=\lim _{N \rightarrow \infty} \frac{1}{N} \sum_{i, j} R(i, j) E_{P_{0}}\left[I C\left(\bar{P}_{0}\right)\left(O_{i}\right) I C\left(\bar{P}_{0}\right)\left(O_{j}\right)\right],
$$


with $R(i, j)=1$ when $F_{i} \cap F_{j} \neq \varnothing$, and $R(i, j)=0$ otherwise. Replacing the unknown components of $\bar{P}_{0}$ in $\operatorname{IC}\left(\bar{P}_{0}\right)$ with corresponding estimates, we then obtained the following estimator $\sigma_{I P T W, N}^{2}$ of $\sigma_{I P T W, 0}^{2}$ :

$$
\sigma_{I P T W, N}^{2}=\frac{1}{N} \sum_{i, j}\left[R(i, j)\left(Y_{i} \frac{\bar{g}_{N}^{*}}{\bar{g}_{N}}\left(A_{i}^{s} \mid W_{i}^{s}\right)-\psi_{I P T W, i, N}\right)\left(Y_{j} \frac{\bar{g}_{N}^{*}}{\bar{g}_{N}}\left(A_{j}^{s} \mid W_{j}^{s}\right)-\psi_{I P T W, j, N}\right)\right]
$$

for $I C\left(\bar{P}_{0}\right)\left(O_{i}^{s}\right)=Y_{i}\left[\bar{g}_{0}^{*} / \bar{g}_{0}\right]\left(A_{i}^{s} \mid W_{i}^{s}\right)-\psi_{0, i}$. We then constructed $95 \%$ CIs as $\psi_{I P T W, N} \pm 1.96 \sigma_{I P T W, N}^{2} / \sqrt{N}$.

\subsection{G-computation estimator}

The G-computation substitution estimator $\psi_{G C O M P, N}=\Psi\left(\bar{Q}_{N}, Q_{W, N}\right)$ for $\psi_{0}$ is based on the un-targeted model $\bar{Q}_{N}$ for the common-in- $i$ conditional expectation of $Y_{i}$, as a function of the summary data $\left(A_{i}^{s}, W_{i}^{s}\right)$. Given stochastic intervention $g^{*}$, the G-computation estimator is obtained as:

$$
\psi_{G C O M P, N}=\frac{1}{N} \sum_{i=1}^{N} \int_{a} \bar{Q}_{N}\left(a_{i}^{s}(a, W), W_{i}^{s}\right) g^{*}(a \mid W)
$$

where $Q_{W, N}$ is a NPMLE that puts mass 1 on observed vector $W$. Evaluation of this estimator is equivalent to the Monte Carlo integration procedure described for the TMLE $\psi_{N}^{*}$ in Section 4.4, except that we use the initial estimator $\bar{Q}_{N}$ for $\bar{Q}_{0}$ instead of its targeted version $\bar{Q}_{N}^{*}$. The asymptotic variance of $\psi_{G C O M P, N}$ was not estimated and no CIs were constructed.

\subsection{Results}

Our simulations compared three different model specification scenarios for $\bar{Q}_{0}$ and $\bar{g}_{0}^{*} / \bar{g}_{0}: "(a) Q$ and $\bar{g}^{*} / \bar{g}$ correct" indicates that the models for both estimators, $\bar{Q}_{0}$ and $\bar{g}_{0}^{*} / \bar{g}_{0}$, were correctly specified; "(b) only $\bar{g}^{*} / \bar{g}$ correct" indicates that the model for the estimator of $\bar{Q}_{0}$ was misspecified, while the model for the estimator of $\bar{g}_{0}^{*} / \bar{g}_{0}$ was specified correctly; finally, "(c) only $Q$ correct" indicates that the model for the estimator of $\bar{Q}_{0}$ was specified correctly, while the model for the estimator of $\bar{g}_{0}^{*} / \bar{g}_{0}$ was misspecified. Figure 1 and Figure 2 present the simulation results for finite sample bias and empirical variance. Bias was plotted as the estimate minus the true parameter value $\left(\psi_{N}\left(g_{p}^{*}\right)-\psi_{0}\left(g_{p}^{*}\right)\right)$, with different stochastic interventions $g_{p}^{*}$ presented on the x-axis as "\% Treated". Overall, our simulation results suggest that TMLE performs well in finite samples with dependent observations. We were able to demonstrate the double robustness property of TMLE, with it being unbiased in each of the three considered scenarios. Our results also indicate that the other two estimators are unbiased for scenario $(a)$, but can perform poorly in alternative scenarios $(b)$ and $(c)$. Overall, we found the IPTW estimator to be the most variable and also most susceptible to near-positivity violations. 
(a) $Q$ and $\bar{g}^{*} / \bar{g}$ correct

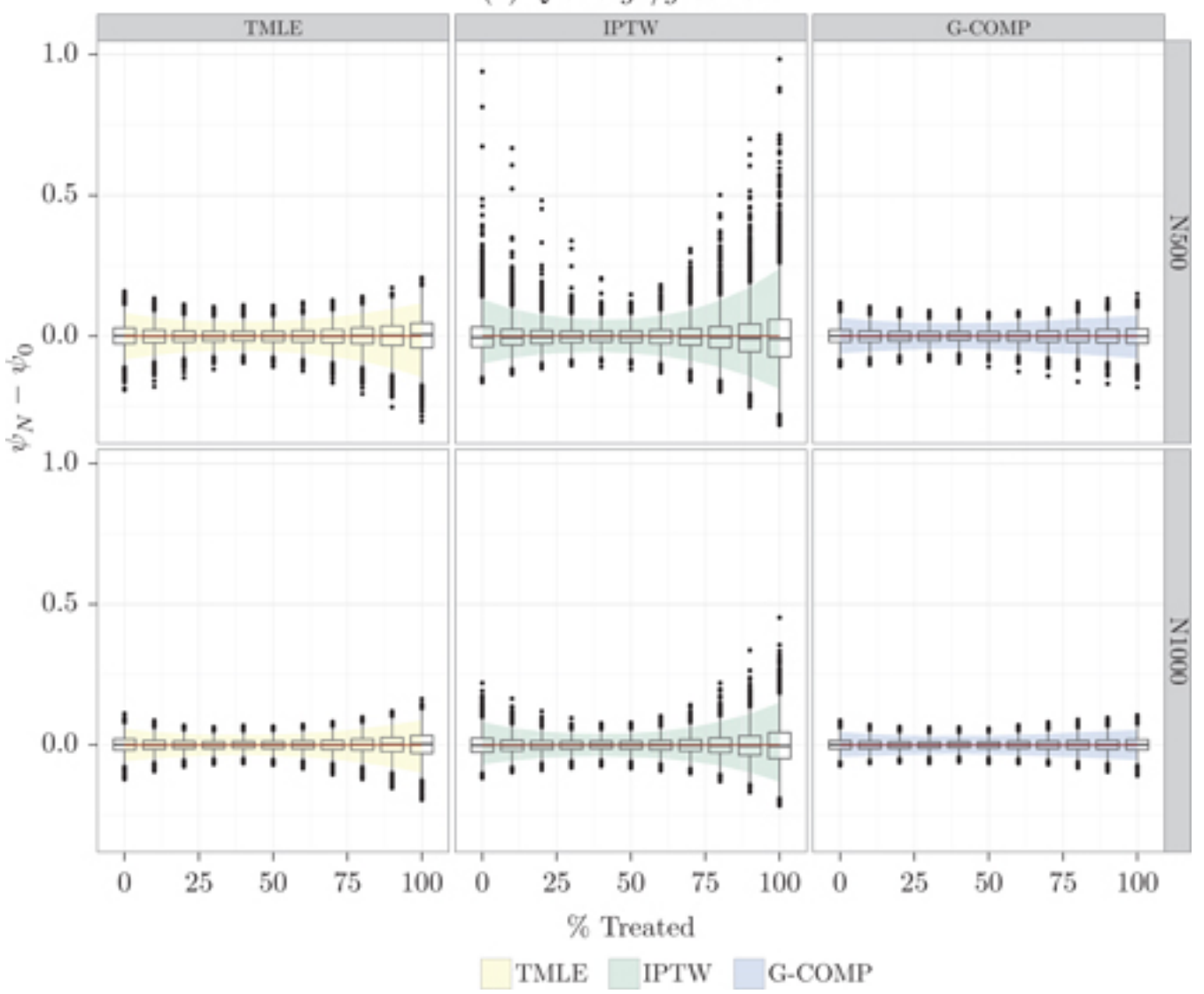

Figure 1 Empirical distributions for TMLE, IPTW and G-COMP, centered at the truth and estimated over 10,000 simulated data sets of size 500 (top row) and size 1,000 (bottom row) for scenario (a) - correctly specified $Q$ and $\bar{g}^{*} / \bar{g}$. Colored ribbons mark the 2.5 th to 97.5 th percentile ranges of the estimands. The centered IPTW estimates outside the range of \pm 1 were removed. 

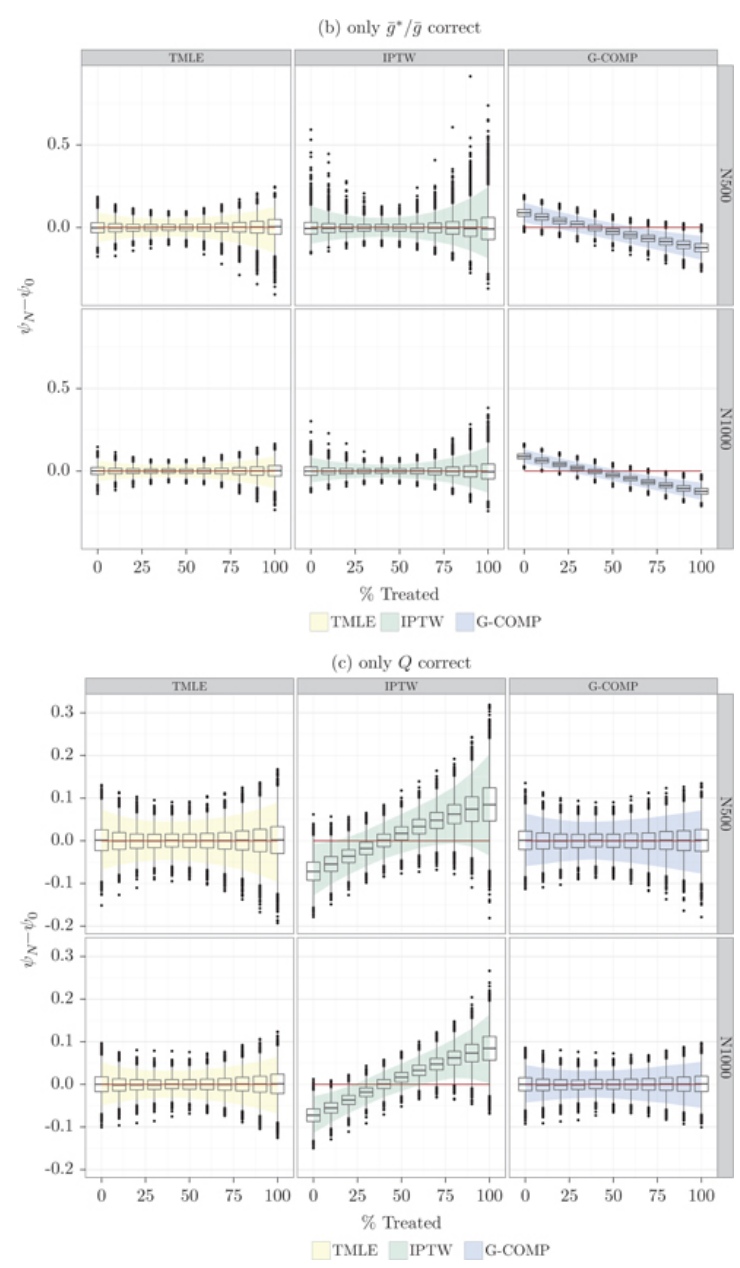

Figure 2 Empirical distributions for TMLE, IPTW and G-COMP, centered at the truth and estimated over 10,000 simulated data sets of size 500 (top row) and size 1,000 (bottom row) for scenarios: (b) - only $\bar{g}^{*} / \bar{g}$ correctly specified; and (c) - only $Q$ correctly specified. Colored ribbons mark the 2.5 th to 97.5 th percentile ranges of the estimands. The centered IPTW estimates outside the range of \pm 1 were removed.

The coverage results are presented in Figure 3, Figure 4 and Figure 5, where we plotted the 95\% CI coverage for various asymptotic variance estimators, along with the mean CI length. We first compared the TMLE coverage of our proposed variance estimator, $\sigma_{N}^{2}$, from Section 6 to the TMLE coverage based on the iid variance estimate $\sigma_{I I D, N}^{2}$ that made no adjustments for correlated observations, i.e., $\sigma_{I I D, N}^{2}$ is the EIC-based variance estimator that assumes data are iid. Our results in Figure 3 indicate that $\sigma_{I I D, N}^{2}$ tended to under-estimate the variance of TMLE, resulting in CIs that were too narrow for both sample sizes. We expect the coverage issues for $\sigma_{I I D, N}^{2}$ to become even more pronounced when the between-unit dependence increases, as may be the case in more realistic network scenarios with units having much higher degrees of connectivity. 

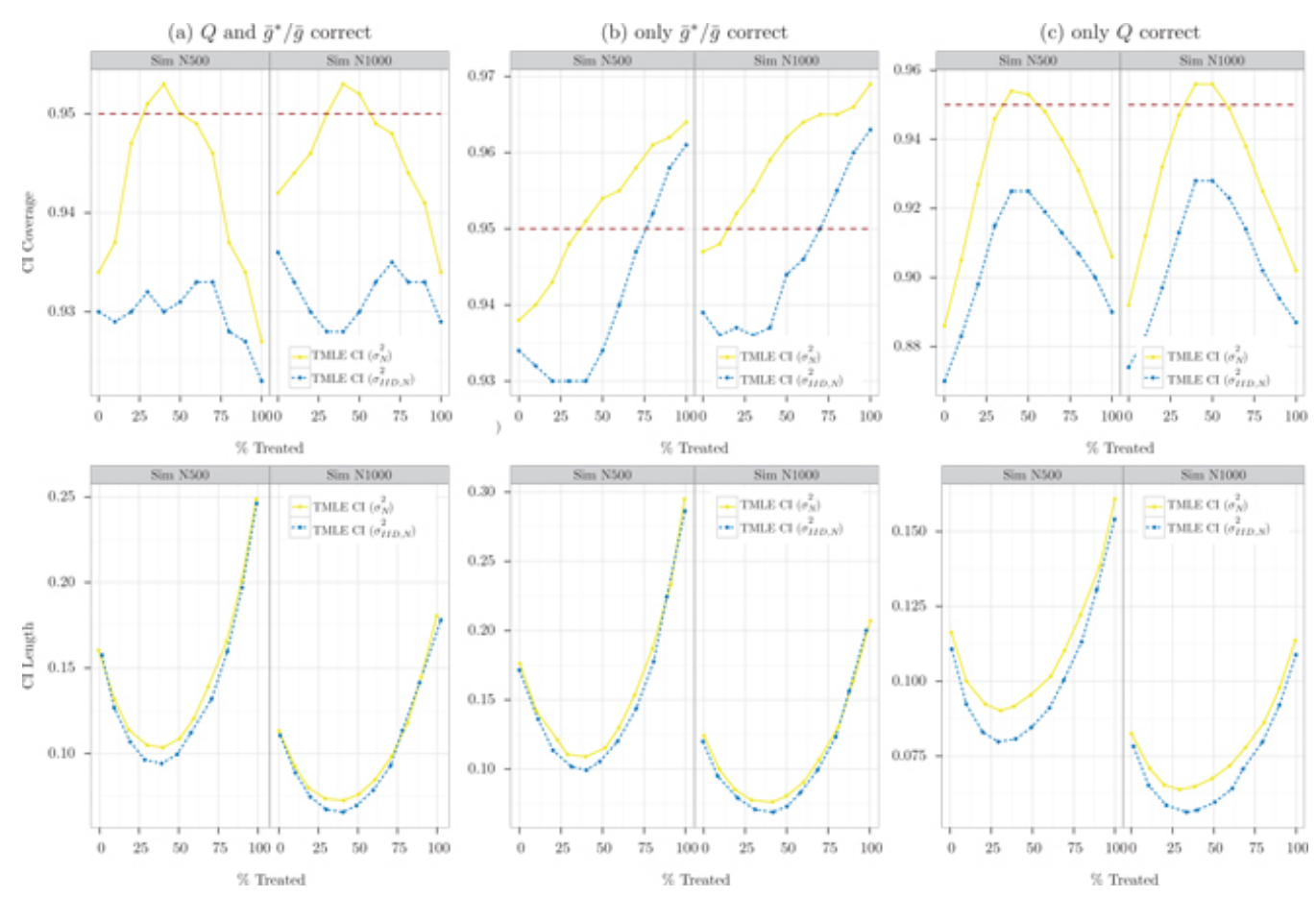

Figure 3 Comparing the $95 \%$ CI coverage (top row) and length (bottom row) for the TMLE using two alternative variance estimates: $\sigma_{N}^{2}$ - variance estimate that correctly adjusts for the dependence between observations; $\sigma_{I I D, N^{-}}^{2}$ iid variance estimate that ignores the dependence between observations. The estimates are obtained from 10,000 simulated data sets of size 500 ('Sim N500') and size 1,000 ('Sim N1000'). Scenarios: (a) - correctly specified $Q$ and $\bar{g}^{*} / \bar{g}$; (b) - only $\bar{g}^{*} / \bar{g}$ correctly specified; and (c) - only $Q$ correctly specified.

In addition, the CIs for our dependent-data variance estimate $\sigma_{N}^{2}$ become conservative when $\bar{Q}_{N}$ was misspecified. The latter result was expected based on the predictions from the semi-parametric efficiency theory for iid data. In Figure 4 we compared the coverage of IPTW with that of TMLE. Finally, we compared the TMLE coverage for our dependent-data variance estimate $\sigma_{N}^{2}$ to the alternative asymptotic variance estimate $\tilde{\sigma}_{N}^{2}$ from van der Laan [9]. The simulation results of this comparison in Figure 5 show nearly identical coverage under correctly specified $\bar{Q}_{N}$. However, when $\bar{Q}_{N}$ is misspecified, the two estimators behaved differently, with $\tilde{\sigma}_{N}^{2}$ showing slightly lower coverage for some sections of the estimated dose response curve. We also note that near positivity violations will generally increase the variability of our estimators. In particular, one would expect the near positivity violations to be more pronounced closer to the tail-ends of the discrete dose response curve $\left\{\psi_{0}\left(g_{p}^{*}\right)\right\}$, namely, for values of $p$ close to 0 or 1 . Indeed, this is also demonstrated in our simulations, where we noted increasing variability of all estimators closer to the edges of the estimated dose response curve, which also contributes to a small drop in coverage. 

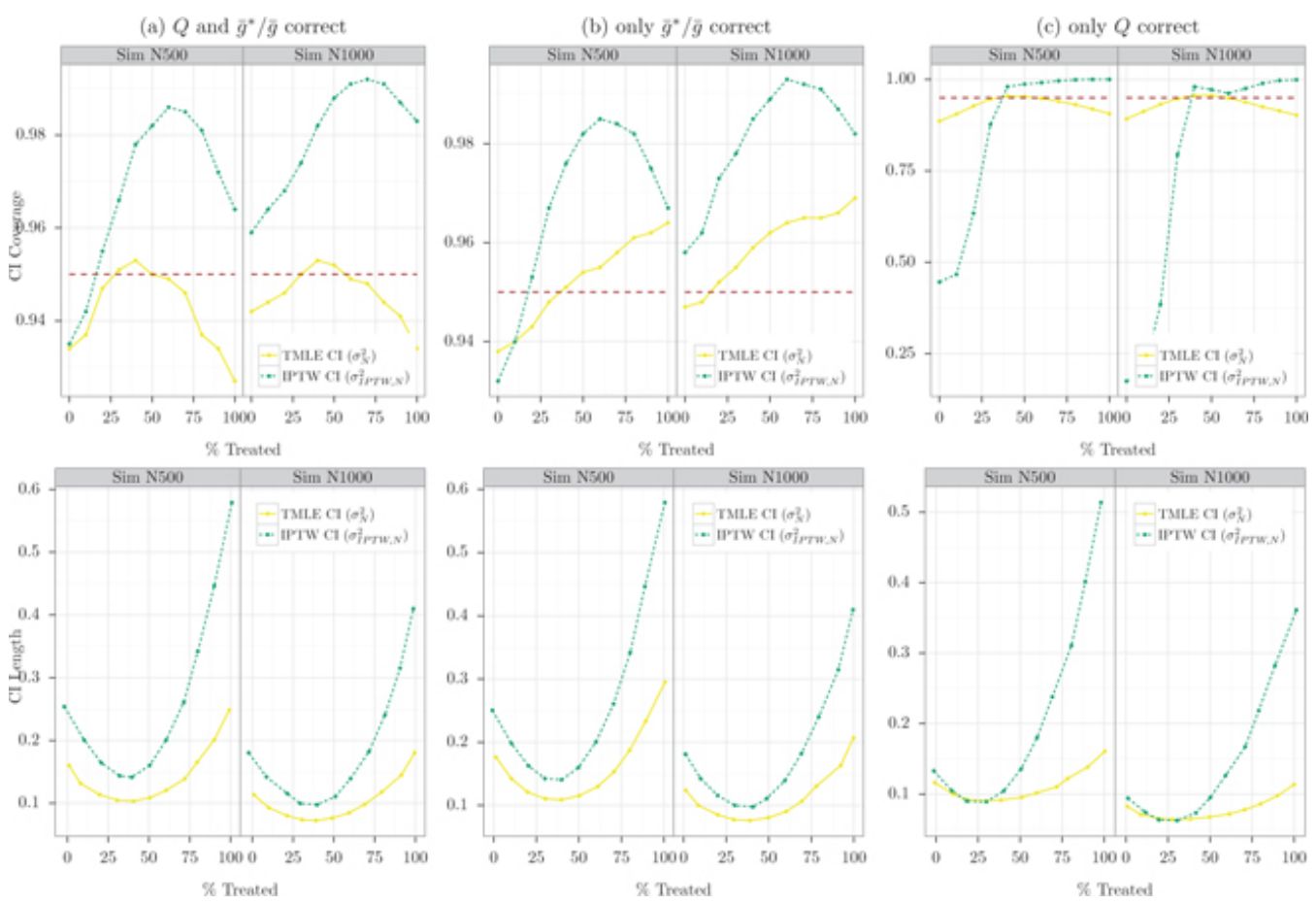

Figure 4 Comparing the $95 \%$ CI coverage (top row) and length (bottom row) for TMLE (variance estimate $\sigma_{N}^{2}$ ) and IPTW (variance estimate $\sigma_{I P T W, N}^{2}$ ). The estimates are obtained from 10,000 simulated data sets of size 500 ('Sim N500') and size 1,000 ('Sim N1000'). Scenarios: (a) - correctly specified $Q$ and $\bar{g}^{*} / \bar{g} ;$ (b) - only $\bar{g}^{*} / \bar{g}$ correctly specified; and (c) - only $Q$ correctly specified.

(a) $Q$ and $\bar{g}^{*} / \bar{g}$ correct
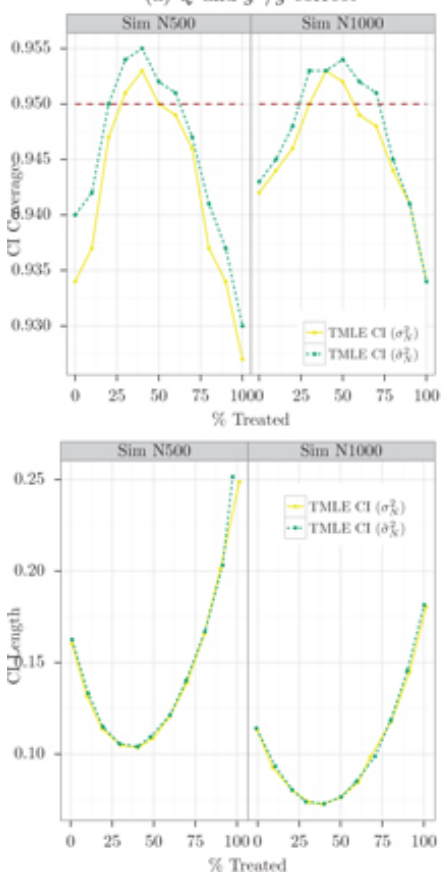

(b) only $\bar{g}^{*} / \bar{g}$ correct
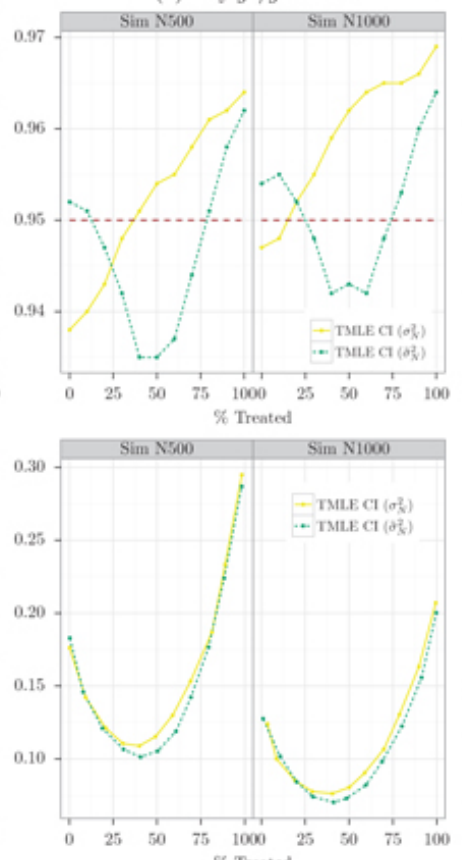

(c) only $Q$ correct
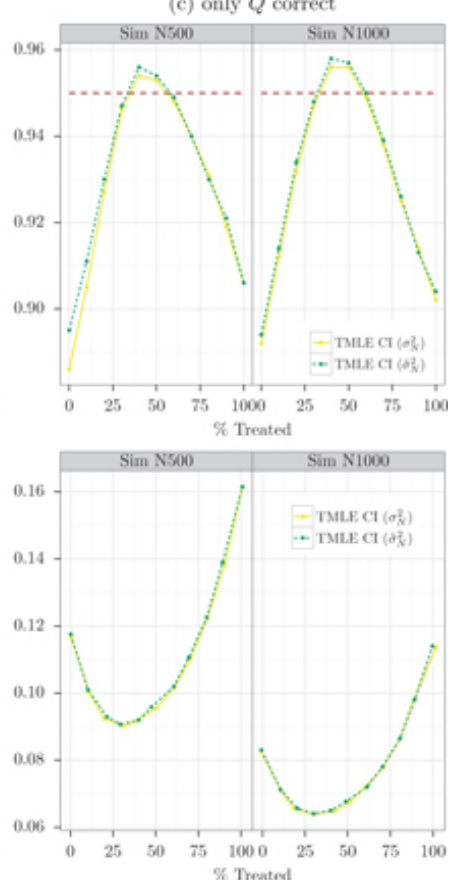

Figure 5 Comparing the $95 \%$ CI coverage (top row) and length (bottom row) of the two TMLE variance estimates, $\sigma_{N}^{2}$ and $\tilde{\sigma}_{N}^{2}$, both of which adjust for the dependence between units, but the latter variance estimate assumes correctly specified $\bar{Q}_{N}$. The estimates are obtained from 10,000 simulated data sets of size 500 ('Sim N500') and size 1,000 ('Sim N1000'). Scenarios: (a) - correctly specified $Q$ and $\bar{g}^{*} / \bar{g}$; (b) - only $\bar{g}^{*} / \bar{g}$ correctly specified; and (c) - only $Q$ correctly specified. 


\section{Intervening on groups of friends and intervening on network}

\subsection{Estimation for an arbitrary collection of stochastic interventions}

We now show that the estimation framework presented thus far can be easily adapted to the estimation of the sample-average treatment effects for an arbitrary collection of $i$-specific stochastic interventions $g_{F_{i}}^{*}$, where each $g_{F_{i}}^{*}$ may intervene on the treatments of $i$ 's friends in addition to intervening on the treatment of $i$ itself. A collection of such interventions involving all units, $\left\{g_{F_{i}}^{*}: i=1, \ldots, N\right\}$, generally cannot be described by a single intervention $g^{*}$ on $A=\left(A_{1}, \ldots, A_{N}\right)$ given $W=\left(W_{1}, \ldots, W_{N}\right)$. For example, consider the problem of estimating the direct average treatment effect in a network of $N$ connected individuals, where we define each $g_{F_{i}}^{*}$ by intervening on a unit-specific treatment, $A_{i}$, setting it to a constant (zero or one), but leave unchanged the distribution of $A_{j}$ for $i$ 's friends $j \in F_{i}$ intact. That is, we assume the intervention for each $A_{j}$ is stochastically sampled from its observed distribution $G_{0}\left(A_{j} \mid W_{j}^{s}\right)$ or instead deterministically set to its observed value $a_{j}$. This type of direct effect parameter has been previously explored in spillover studies, for example, in the study of the effects of deworming among rural Kenyan primary schools by Miguel and Kremer [46] and in its replication study by Davey et al. [47]. We are interested in estimation of the following target parameter,

$$
\psi_{0}=\Psi\left(P_{0}^{N}\right)=\frac{1}{N} \sum_{i=1}^{N} E_{q_{0}, g_{F_{i}}^{*}}\left[Y_{i}^{g_{F_{i}}^{*}}\right]
$$

defined as the average of expectations of $Y_{i}^{g_{F_{i}}^{*}}$, where each outcome $Y_{i}^{g_{F_{i}}^{*}}$ is generated under the $i$-specific postintervention distribution that replaces the observed treatment allocation for $i$ and $j \in F_{i}$ with $g_{F_{i}}^{*}$ as just described. Clearly the collection of such $i$-specific interventions across all $N$ units is incompatible with a single joint stochastic intervention $g^{*}$ on $A$ given $W$, since the intervention $g_{F_{j}}^{*}$ for $j \neq i$ and $j \in F_{i}$ requires setting $A_{j}$ to a constant one or zero, while the intervention $g_{F_{i}}^{*}$ requires that $A_{j}$ is randomly sampled from $g_{0}$ or is set to $a_{j}$. Nonetheless, this target parameter $\psi_{0}$ remains well-defined with respect to a collection $\left\{g_{F_{i}}^{*}: i=1, \ldots, N\right\}$, and we may apply the same arguments as in Section 3.1, noting that $\psi_{0}$ can be equivalently written as:

$$
\begin{aligned}
\psi_{0} & =\frac{1}{N} \sum_{i=1}^{N} \int_{a, w} \bar{Q}_{0}\left(a_{i}^{s}(a, w), w_{i}^{s}(w)\right) g_{F_{i}}^{*}(a \mid w) q_{W, 0}(w) d \mu(a, w) \\
& =\frac{1}{N} \sum_{i=1}^{N} \int_{a^{s}, w^{s}} \bar{Q}_{0}\left(a^{s}, w^{s}\right) h_{i, 0}^{*}\left(a^{s}, w^{s}\right) d \mu\left(a^{s}, w^{s}\right) \\
& =\int_{a^{s}, w^{s}} \bar{Q}_{0}\left(a^{s}, w^{s}\right) \bar{h}_{0}^{*}\left(a^{s}, w^{s}\right) d \mu\left(a^{s}, w^{s}\right),
\end{aligned}
$$

where $h_{i, 0}^{*}\left(g_{F_{i}}^{*}, q_{W, 0}\right)$ is the density determined by $g_{F_{i}}^{*}(a \mid w), q_{W, 0}$ and the $i$-specific summary measures $a_{i}^{s}(a, w), w_{i}^{s}(w)$, and $\bar{h}_{0}^{*}$ is a mixture of $h_{i, 0}^{*}$, defined as $\bar{h}_{0}^{*}\left(a^{s}, w^{s}\right):=1 / N \sum_{i=1}^{N} h_{i, 0}^{*}\left(a^{s}, w^{s}\right)$. We also note that when $(W, A)$ are discrete, one obtains:

$$
h_{i, 0}^{*}\left(a^{s}, w^{s}\right)=\int_{a, w} I\left(a_{i}^{s}(a, w)=a^{s}, w_{i}^{s}(w)=w^{s}\right) g_{F_{i}}^{*}(a \mid w) q_{W, 0}(w) d \mu_{a, w}(a, w) .
$$

Thus, this new target parameter $\Psi\left(P_{0}^{N}\right)$ can still be represented by an equivalent mixture mapping $\bar{\Psi}\left(\bar{P}_{0}\right)$ from Theorem Theorem 3.1 and hence, the efficient influence curve of this new $\Psi\left(P_{0}^{N}\right)$ is given by the same $\bar{D}$ from Theorem Theorem 3.3. In the above, we also assumed that such $i$-specific densities $h_{i, 0}^{*}\left(a^{s}, w^{s}\right)$ are welldefined with respect to some common dominating measure $\mu_{a, w}$, with $\bar{h}_{0}^{*}$ being factorized as $\bar{h}_{0}^{*}\left(a^{s}, w^{s}\right)=$ $\bar{g}_{0}^{*}\left(a^{s} \mid w^{s}\right) \bar{q}_{W, 0}\left(w^{s}\right)$, which provides the definition of $\bar{g}_{0}^{*}$. Hence, the TMLE framework from Section 4 can be directly applied to estimation of these types of parameters, with the only modification that we now require that each $i$-specific summary $A_{i}^{* s}$ is sampled conditionally from its $i$-specific intervention $g_{F_{i}}^{*}$ on $A$ given $W$. In particular, we use Lemma Lemma 4.1 to obtain a reasonable approximation for $\bar{g}_{0}^{*}$ by modifying it's direct estimator from Section 4.3 in the following manner: First, obtain a large simulated dataset of $i$-specific summaries $\left(A_{i}^{* s}, W_{i}^{s}\right)$, for $i=1, \ldots, N$, where each $A_{i}^{* s}$ is derived by sampling $\left(A^{g_{F_{i}}^{*}}, W\right)$ from $\left(g_{F_{i}}^{*}, Q_{W, N}\right)$ and then 
applying the summary measure mapping $a_{i}^{S}\left(A^{g_{F_{i}}^{*}}, W\right)$. Next, fit an estimator $\bar{g}_{N}^{*}$ of $\bar{g}_{0}^{*}$ by treating the simulated sample $\left(A_{i}^{* s}, W_{i}^{s}\right)$ as iid, applying the same maximum likelihood-based approach as earlier. Similarly, the Monte Carlo evaluation step for the TMLE $\psi_{N}^{*}$ from Section 4.4 is modified to take into account these $i$-specific interventions $g_{F_{i}}^{*}$. That is, instead of sampling $\left(A^{*}, W\right)$ from $g^{*}$, each Monte Carlo iteration $j$ now consists of sampling $A_{j}^{g_{F_{i}}^{*}}=\left(A_{j, 1}^{g_{F_{i}}^{*}}, \ldots, A_{j, N}^{g_{F_{i}}^{*}}\right)$ from $g_{F_{i}}^{*}(a \mid W)$, for each $i=1, \ldots, N$, conditional on the observed $W$, with a resulting dataset of $N$ summary observations $\left(A_{j, i}^{* s}, W_{i}^{s}\right)$ constructed by applying the $i$-specific mappings $A_{j, i}^{* s}:=a_{i}^{s}\left(A_{j}^{g_{F_{i}}^{*}}, W\right)$, for $i=1, \ldots, N$.

\subsection{Estimation for interventions on the network structure}

\section{Overview. Observed network data}

We note that our framework can be also applied to estimation of the effect of intervening on the network structure. Suppose that we observe at baseline some initial network $F^{0}=\left(F_{1}^{0}, \ldots, F_{N}^{0}\right)$ for the community of $N$ connected units and we are treating $F^{0}$ as fixed. As before, we are assuming that the maximum number of friends for each unit (i.e., the dimensionality of each $F_{i}^{0}$ ) is bounded by some constant $K$ that doesn't depend on $N$. We then collect data on $N$ baseline covariates $W=\left(W_{1}, \ldots, W_{N}\right)$, followed by a random draw of another network profile $F=\left(F_{1}, \ldots, F_{N}\right)$ and the outcomes $Y=\left(Y_{1}, \ldots, Y_{N}\right)$, where each $F_{i}$ is now based on the initial network $F_{i}^{0}$. Thus, the observed data on $N$ connected units is given by $O=\left(F^{0}, W, F, Y\right)$ and we assume the exposure for each unit $i$ is given by the set $F_{i}$. Since we are interested in interventions which will modify the observed network profile $F$ (e.g., adding or removing some friends in each $F_{i}$ ) it is natural to allow $F_{i}$ to be random, but driven by $i$ 's own covariates and the covariates of $i$ 's friends from $F_{i}^{0}$. Thus, we assume that the $i$-specific conditional distribution $G_{F_{i}, 0}$ for $F_{i}$ given $\left(F^{0}, W\right)$ only depends on the initial network offset $F_{i}^{0}$ and the baseline covariates $\left(W_{i}, W_{j}: j \in F_{i}^{0}\right)$. Furthermore, we assume its conditional density $g_{F_{i}, 0}\left(F_{i} \mid F^{0}, W\right)$ is well-defined. We also assume that $Q_{Y, 0}$, the common-in- $i$ conditional distribution of $Y_{i}$ given $W$, depends only on $\left(W_{i}, W_{j}: j \in F_{i}\right)$, i.e., units $j$ from this newly drawn friend set $F_{i}$.

\section{Network interventions and target parameter}

We follow the framework outlined in Section 2 and define the intervention on a network profile $F$ as a usersupplied density $g^{*}\left(F^{*} \mid W\right)$ that replaces the observed conditional density $g_{0}(F \mid W)$, where we also assumed that the initial network offset $F^{0}$ is included in $W$. Alternatively, we could also follow Section Section 8.1 and define our intervention as a collection of the user-supplied $i$-specific densities $\left\{g_{F_{i}}^{*}: i=1, \ldots, N\right\}$, where each $g_{F_{i}}^{*}\left(F_{i}^{*} \mid W\right)$ replaces the true $i$-specific density $g_{F_{i}, 0}\left(F_{i} \mid W\right)$. As noted in Section 8.1, a collection of such $i$-specific stochastic interventions generally cannot be described by a single multivariate intervention $g^{*}$ on $F=\left(F_{1}, \ldots, F_{N}\right)$ given $W=\left(W_{1}, \ldots, W_{N}\right)$ and may result in an incompatible network intervention. Nonetheless, these are still welldefined interventions and we note that the target parameter indexed by such $i$-specific interventions $g_{F_{i}}^{*}\left(F_{i}^{*} \mid W\right)$ is still well-defined. We also note that the types of interventions we will consider will generally use the current network sets $F_{i}$ as inputs, to produce intervened network sets $F_{i}^{*}$. Therefore, we are concerned here with stochastic interventions which depend on the current sets $F_{i}$. Even if the intervention itself is a deterministic function of $F_{i}$ (e.g., always remove the first friend), it is still stochastic by the nature of its dependence on $F_{i}$.

As a motivating example, consider an intervention defined for $i=1, \ldots, N$ that removes certain friends $j \in F_{i}$ of each unit $i$ when $\delta\left(W_{j}\right) \geq r$, where $r$ is some pre-defined cutoff value and $\delta\left(W_{j}\right)$ is some user-defined function mapping $W_{j}$ in $\mathbb{R}$ (e.g., $\delta\left(W_{j}\right)$ characterizes the baseline "risk-profile" of $j$ ). This intervention defines the post-intervention distribution that replaces $Q_{Y, 0}\left(W_{j}: j \in F_{i}\right)$, i.e., the observed conditional distribution of $Y_{i}$ given $W$, with a new distribution $Q_{Y, 0}\left(W_{j}: j \in F_{i}^{*}\right)$, where $F_{i}^{*}$ is the intervened friend set such that the unit $k \in F_{i}^{*}$ only if $k \in F_{i}$ and $\delta\left(W_{k}\right)<r$. We now define our statistical parameter as the sample-average of the expected outcomes under the $i$-specific post-intervention distributions that replace each observed network allocations $g_{F_{i}, 0}$ with such intervention densities $g_{F_{i}}^{*}$ :

$$
\Psi\left(P_{0}^{N}\right)=\frac{1}{N} \sum_{i=1}^{N} E_{q_{0}, g_{F_{i}}^{*}}\left[Y_{i}^{g_{F_{i}}^{*}}\right] .
$$

Statistical model and dimension reduction assumptionsWe have described how the interventions on the networks sets $F_{i}$ fit within our previously outlined framework in Section 2, where $F_{i}$ defines the exposure for each 
unit $i$. Our next step is concerned with dimensionality reduction, where we define the appropriate summary measures $\left(W_{i}^{s}, A_{i}^{s}\right)$, for $i=1, \ldots, N$, and then model conditional distribution of $Y_{i}$ as a fixed-dimensional function of $\left(W_{i}, A_{i}^{s}\right)$. In order to be able to intervene on $F_{i}$ we have to assume that the conditional distribution $Q_{Y, 0}$ depends on all $W$ through an $N$ dimensional set $\left(W_{j} I\left(j \in F_{i}\right): j=1, \ldots, N\right)$. That is, we have so far assumed that $Q_{Y, 0}$ is a function of $\left(W_{i}, W_{j}: j \in F_{i}\right)$. Replacing $F_{i}$ with the intervened friend set $F_{i}^{*}$ implies that $Q_{Y, 0}$ becomes a function of $\left(W_{i}, W_{j}: j \in F_{i}^{*}\right)$. In that sense, $Y_{i}$ depends on all $W$, except that for most $j$, we have $I\left(j \in F_{i}\right)=0$ and hence $W_{j}$ makes no real contribution to $Q_{Y, 0}$, unless $j \in F_{i}$. In summary, we change the dependence of $Y_{i}$ on particular $W_{j}$ in $W$ by changing the composition of the set $F_{i}$ and we only intervene on the way $Y_{i}$ may depend on a particular $W_{j}$ through indicators $I\left(j \in F_{i}\right)$, for $j=1, \ldots, N$. This also implies that the conditional distribution of the outcome $Y_{i}$ is now truly a function of the entire $N$ dimensional set $W=\left(W_{1}, \ldots, W_{N}\right)$, making estimation of $Q_{Y, 0}$ particularly challenging. Thus, we first need to make additional simplifying assumptions which would allow us to estimate $Q_{Y, 0}$.

For convenience, we now assume that $i \in F_{i}^{0}, i \in F_{i}$ and $i \in F_{i}^{*}$, for all $i=1, \ldots, N$ (i.e., $i$ is always connected to itself). Assume that the $i^{\prime}$ s network draw $F_{i}$ is always a finite dimensional augmentation of the initial network offset $F_{i}^{0}$. That is, the set of possible realizations of $F_{i}$ is restricted to be within some close proximity of $F_{i}^{0}$. We note that the network profile $F$ can be viewed as an $N \times N$ adjacency matrix of indicators and our assumptions imply that rather then allowing $F$ to be any possible realization of an $N \times N$ adjacency matrix, we restrict $F$ to finite-dimensional perturbations of matrix $F^{0}$, allowing $F$ to only change locally as a function of $F_{0}$ and $W$. For example, rather than allowing $F_{i}$ to draw any new friend $j \in\{1, \ldots, N\} \backslash i$, one may assume that $F_{i}$ is restricted to drawing a new friend $j$ only when $j$ is in a set $F_{i}^{0+}:=\cup_{j \in\left\{F_{i} \cup i\right\}} F_{j}^{0}$. In this case we are assuming that in the new network realization $F_{i}$ can only add friend $j$ if $i$ and $j$ had at least one friend in common at baseline (i.e., there was at most 2 nd degree of connectivity between $i$ and $j$ ). Such an assumption implies that $F_{i}$ is no longer of dimension $N$, but is rather of a fixed dimension that only depends on $K$. The set of possible network interventions on $F_{i}$, namely, each intervened $F_{i}^{*}$, is now similarly restricted to realizations of the same finite-dimensional set $F_{i}^{0+}$.

Having defined $F_{i}$ as a realization of the finite dimensional set $F_{i}^{+}$, we define the unit's exposure $A_{i}$, for $i=1, \ldots, N$, as a finite-dimensional set of indicators $\left(I\left(j \in F_{i}\right): j \in F_{i}^{0+}\right)$, namely, each $A_{i}$ is a binary vector of the common-in- $i$ dimension $K^{+}$, with each non-zero entry in $A_{i}$ denotes which units in $F_{i}^{0+}$ are actual friends of $i$. The $i$-specific network intervention $A_{i}^{*}$ can be defined by directly intervening on the indicators $I\left(j \in F_{i}\right)$ in $A_{i}$. We define the baseline summary measure $W_{i}^{s}:=w_{i}^{s}(W)$ as a finite $\operatorname{dimensional~set}\left(W_{j}: j \in F_{i}^{0+}\right)$, for $i=1, \ldots, N$. Additionally, we define the exposure summary measure $a_{i}^{s}\left(A_{i}, W_{i}^{s}\right)$ that depends on $\left(A_{i}, W_{i}^{s}\right)$ only as a function of the set $\left(W_{j} I\left(j \in F_{i}\right): j \in F_{i}^{0+}\right)$, i.e.,

$$
A_{i}^{s}:=a_{i}^{s}\left(A_{i}, W_{i}^{s}\right)=a_{i}^{s}\left(W_{j} I\left(j \in F_{i}\right): j \in F_{i}^{0+}\right),
$$

for $i=1, \ldots, N$, and we assume each $a_{i}^{s}(\cdot)$ maps into $\mathbb{R}^{d}$, for a common-in- $i$ dimension $d$. Next, we model the conditional probability of the outcome $Y_{i}$ as a common-in- $i$ function $Q_{Y, 0}$ that depends on $(A, W)$ only as a function of the summary $A_{i}^{s}$, for $i=1, \ldots, N$. Note that the crucial assumption that $a_{i}^{S}(\cdot)$ is a function of $\left(W_{j} I\left(j \in F_{i}\right): j \in F_{i}^{0+}\right)$ allows us to take full advantage of the dimensionality reduction due to $a_{i}^{s}(\cdot)$ and still define the target parameters which actually correspond to the effects of intervening on the observed network realization $F$. We may also assume that $Y_{i}$ depends on some common-in- $i$ summary $\tilde{w}^{s}\left(W_{j}: j \in F_{i}^{0+}\right)=\tilde{w}^{s}\left(W_{i}^{s}\right)$ that is unrelated to the new network draw $F_{i}$, and hence would not be affected by an intervention $F_{i}^{*}$ on $F_{i}$. Note that such modeling restrictions on $F_{i}$ now make it possible to estimate our parameter $\Psi\left(P_{0}^{N}\right)$.

With a slight abuse of notation, we use $g_{F_{i}, 0}\left(A_{i} \mid W\right)$ to denote the conditional density of $A_{i}$ given $W$, and similarly, we use $g_{F_{i}}^{*}\left(A_{i}^{*} \mid W\right)$ to denote the $i$-specific stochastic intervention on $A_{i}$, implied by $F_{i}^{*}$. We also note that due to our modeling assumptions for the conditional distribution of $F_{i}$ given $\left(F^{0}, W\right)$, we have that $g_{F_{i}, 0}\left(A_{i} \mid W\right)=g_{F_{i}, 0}\left(A_{i} \mid W_{i}^{s}\right)$ and $g_{F_{i}}^{*}\left(A_{i} \mid W\right)=g_{F_{i}}^{*}\left(A_{i} \mid W_{i}^{s}\right)$, which also leads to the following representation of our target parameter: 


$$
\begin{aligned}
\Psi\left(P_{0}^{N}\right) & =\frac{1}{N} \sum_{i=1}^{N} E_{Q_{W_{i}^{s}, 0}}\left[E_{g_{F_{i}}^{*}}\left[\bar{Q}_{0}\left(a_{i}^{s}\left(A_{i}^{*}, W_{i}^{s}\right), \tilde{w}^{s}\left(W_{i}^{s}\right)\right) \mid W_{i}^{s}\right]\right] \\
& =\frac{1}{N} \sum_{i=1}^{N} \int_{a_{i}, w_{i}^{s}} \bar{Q}_{0}\left(a_{i}^{s}\left(a_{i}, w_{i}^{s}\right), \tilde{w}^{s}\left(w_{i}^{s}\right)\right) g_{F_{i}}^{*}\left(a_{i} \mid w_{i}^{s}\right) q_{W_{i}^{s}, 0}\left(w_{i}^{s}\right) d \mu\left(a_{i}, w_{i}^{s}\right) \\
& =\frac{1}{N} \sum_{i=1}^{N} \int_{a^{s}, w^{s}} \bar{Q}_{0}\left(a^{s}, \tilde{w}^{s}\left(w^{s}\right)\right) h_{i, 0}^{*}\left(a^{s}, w^{s}\right) d \mu\left(a, w^{s}\right) \\
& =\int_{a, w^{s}} \bar{Q}_{0}\left(a^{s}, \tilde{w}^{s}\left(w^{s}\right)\right) \bar{h}_{0}^{*}\left(a^{s}, w^{s}\right) d \mu\left(a^{s}, w^{s}\right) \\
& =\int_{a, w^{s}} \bar{Q}_{0}\left(a^{s}, \tilde{w}^{s}\left(w^{s}\right)\right) \bar{g}_{0}^{*}\left(a^{s} \mid w^{s}\right) \bar{q}_{W, 0}\left(w^{s}\right) d \mu\left(a^{s}, w^{s}\right) \\
& =E_{\bar{q}_{W, 0}}\left[E_{\bar{g}_{0}^{*}}\left[\bar{Q}_{0}\left(\bar{A}^{s}, \tilde{w}^{s}\left(\bar{W}^{s}\right)\right) \mid \bar{W}^{s}\right]\right] .
\end{aligned}
$$

This shows that the new target parameter $\Psi\left(P_{0}^{N}\right)$ can be represented by an equivalent mixture mapping $\bar{\Psi}\left(\bar{P}_{0}\right)$ from Theorem Theorem 3.1. Consequently, the efficient influence curve of this new $\Psi\left(P_{0}^{N}\right)$ is given by the same $\bar{D}$ from Theorem Theorem 3.3. It follows that the estimation procedure described in Section 4 remains unchanged when estimating this new parameter $\Psi\left(P_{0}^{N}\right)$. As before, we also assume that the $i$-specific densities $\bar{h}_{0}^{*}\left(a^{s}, w^{s}\right)$ are well-defined with respect to some common dominating measure, and that $\bar{h}_{0}^{*}$ can be factorized as $\bar{h}_{0}^{*}\left(a^{s}, w^{s}\right)=$ $\bar{g}_{0}^{*}\left(a^{s} \mid w^{s}\right) \bar{q}_{W}\left(w^{s}\right)$.

As we describe in an example below, given a particular context, one might assume that the summary measures $A_{i}^{s}$ are of lower dimensionality than the identity mapping $\left(W_{j} I\left(j \in F_{i}\right): j \in F_{i}^{0+}\right)$. For instance, one may be able to define some low-dimensional summaries of $\left(I\left(j \in F_{i}\right) W_{j}: j \in F_{i}^{0+}\right)$, which incorporate various features of unit $i$ 's network and the covariates of $i$ 's friends. One then has to assume that the conditional outcome model $Q_{Y, 0}$ for each $Y_{i}$ depends only on such low-dimensional features. Furthermore, if $a_{i}^{Y}(\cdot)$ and $\tilde{w}^{\mathcal{S}}(\cdot)$ depend only on some subset of $\left(W_{j}: j \in F_{i}^{0+}\right)$, then $W_{i}^{s}$ can be also redefined as a summary of lower dimension that only includes the subset of $\left(W_{j}: j \in F_{i}^{0+}\right)$ or the specific features of this subset. The direct estimation of the conditional mixtures $\bar{g}_{0}$ and $\bar{g}_{0}^{*}$ is then performed conditional on this lower-dimensional summary $W_{i}^{s}$.

Example Suppose that we gather some initial data on a network of sexual partners $\left(F^{0}=F_{1}^{0}, \ldots, F_{N}^{0}\right)$ in a community-based observational study of HIV risk factors. For each unit $i$, we measure the baseline covariates, $W_{i}$, which may include baseline HIV infection status $\left(H_{i}\right)$ along with various risk factors. We also assume that after some period of time the network of sexual partners on each unit was measured again. This new network realization for unit $i$ is denoted as set $F_{i}$ and it defines our exposure of interest. Suppose that several months later data was collected on the binary outcome $Y_{i}$ which indicated whether subject $i$ contracted HIV during the follow-up period. Our scientific question of interest is to determine the expected incidence of HIV under a hypothetical intervention on the network of sexual partners of each unit $i$. We note that our dimension reduction assumptions imply that the set of possible realizations of $i$ 's partners in a new network draw $F_{i}$ is restricted to units who were already connected to $i$ at baseline through a common partner, the set we previously denoted as $F_{i}^{0+}$. The exposure $A_{i}$ is defined as a vector of indicators $I\left(j \in F_{i}\right)$ based on the set of all possible partner realizations $j \in F_{i}^{0+}$. We now assume that $Q_{Y, 0}$, the conditional probability of unit $i$ contracting HIV, depends on $i^{\prime} \mathrm{s}$ baseline covariates $\left(W_{i}\right)$ and the total number of $i$ 's current partners $\left(n F_{i}:=\sum_{j=1}^{K^{+}} A_{i}(j)\right)$. We also assume that $Q_{Y, 0}$ depends on the covariates of $i^{\prime}$ s current partners in $A_{i}$ only as a function of a lower-dimensional summary measure. For example, we suppose that the risk of contracting HIV for unit $i$ also depends on the proportion of the total number of $i$ 's partners who had HIV at baseline $\left(p H_{i}:=\left(1 / n F_{i}\right) \sum_{j \in F_{i}} H_{j}\right)$, as well as the proportion of $i$ 's partners with high-risk as determined by $\delta\left(W_{j}\right)>r_{1}$, i.e, the summary $p R_{i}=\left(1 / n F_{i}\right) \sum_{j \in F_{i}} I\left(\delta\left(W_{j}\right)>r\right)$, where $\delta(\cdot)$ maps the covariates in $W_{j}$ into a real line. We now suppose that the exposure summary is defined as $A_{i}^{s}:=\left(n F_{i}, p H_{i}, p R_{i}\right)$, and the baseline summary is defined as $W_{i}^{s}:=\left(W_{i}, \delta\left(W_{j}\right): j \in F_{i}^{0+}\right)$. We also consider individual interventions $g_{F_{i}}^{*}$ on $F_{i}$ that replace the observed partners of $i$ in $F_{i}$ with another set of partners $F_{i}^{*}$. For example, we consider the $i$-specific intervention $g_{F_{i}}^{*}$ that decreases the total number of $i$ 's partners by stochastically removing some $j \in F_{i}$, where this probability of removing a partner $j \in F_{i}$ can be a function of $j^{\prime}$ s baseline risk-profile $\delta\left(W_{j}\right)$. Such an intervention $g_{F_{i}}^{*}$ implies a new exposure set $A_{i}^{*}$ and an intervention-specific summary $A_{i}^{* s}:=\left(n F_{i}^{*}, p H_{i}^{*}, p R_{i}^{*}\right)$, where $n F_{i}^{*}:=\sum_{j=1}^{K^{+}} A_{i}^{*}(j), p H_{i}^{*}:=\left(1 / n F_{i}^{*}\right) \sum_{j \in F_{i}^{*}} H_{j}$ and $p R_{i}^{*}:=\left(1 / n F_{i}^{*}\right) \sum_{j \in F_{i}^{*}} I\left(\delta\left(W_{j}\right)>r\right)$. One can now directly apply the TMLE framework from Section 4 to estimate the sample-average of the expected outcomes $Y_{i}$ under such network interventions $g_{F_{i}^{*}}$, for $i=1, \ldots, N$. In 
particular, we first need to estimate the conditional mixtures $\bar{g}_{0}\left(A_{i}^{s} \mid W_{i}^{s}\right)$ and $\bar{g}_{0}^{*}\left(A_{i}^{*} \mid W_{i}^{s}\right)$ using the direct estimation approach, which then allows us to evaluate the clever covariate based on $N$ predictions $\left[\bar{g}_{N}^{*} / \bar{g}_{N}\right]\left(A_{i}^{s} \mid W_{i}^{s}\right)$. We then proceed to fit a common-in- $i$ initial model $\bar{Q}_{N}$ for the regression of $Y_{i}$ given $\left(A_{i}^{s}, W_{i}\right)$, followed by the TMLE update $\bar{Q}_{N}^{*}$ for $\bar{Q}_{N}$.

\section{Discussion}

In this paper we describe a practical application of the TMLE framework towards the goal of estimation of the sample-average treatment effects among possibly dependent units. Our first objective was to assume a realistic semi-parametric data generating model, which reflected the types of between-unit dependence one may encounter in real-life observational network study, for example, when the study units are connected via a social or geographical network. Our approach included a number of statistical assumptions, such as, the assumption of a certain conditional independence of outcomes and the assumption of fixed-dimension summary measures, which allowed us to perform estimation and inference under potential network dependence. Having defined our semi-parametric statistical model $\mathcal{M}$, we also defined our target of estimation $\Psi\left(P^{N}\right)$ as a mapping from the joint distribution $P^{N}$ on $N$ connected units, for $P^{N} \in \mathcal{M}$. We then showed in Section 3.1 Section 3.1 that this target parameter depends on the joint distribution of the data only as a function of the mixture distribution $\bar{P}$, where $\bar{P}$ was given as a mixture of the unit-specific components $P_{i}$ of $P^{N}$. Such mixture mapping representation implied that our estimation problem was reduced to the problem of estimating the relevant factors of the mixture $\bar{P}$. We have argued that such dependent-data parameters can then be estimated by simply ignoring the dependence between connected observations (e.g., Lemma Lemma 4.1 Lemma 4.1), suggesting that an entire class of the iid data estimators, such as the iid TMLE algorithm we described in this paper, may be applied to estimation problems with potential network dependence. That is, we presented the dependent-data TMLE from van der Laan [9] as an iid data algorithm, with an unusual caveat that our stochastic intervention of interest $\bar{g}_{0}^{*}$ is dependent on the true distribution of the observed data. We also provided a simple estimator of the true asymptotic variance of the TMLE that was shown to be consistent for the model with independent baseline covariates. This variance estimator takes into account the known network structure, making adjustments for correlated EIC contributions of connected units. We also discussed the difficulty and proposed a few ad-hoc approaches for estimating the variance in a model with weakly dependent baseline covariates. Finally, we described an alternative approach for inference that conditions on the baseline covariates - an approach that results in a consistent variance estimate even for a model with weakly dependent baseline covariates. We also assessed the validity of our proposed inferential framework with a finite sample network simulation study. In particular, the finite sample performance of our proposed TMLE was compared to the parametric G-computation estimator and the IPTW estimator. Lastly, we assessed the finite sample coverage of our estimated asymptotic confidence intervals, e.g., comparing our dependent-data inference to inference that completely ignores the dependence among units. We observed that the latter approach to inference resulted in a coverage that was consistently below the nominal $95 \%$. We also expect this coverage to become increasingly worse as one moves towards more realistic network scenarios characterized by denser networks and higher levels of between-unit dependence. However, we leave this topic to be explored in future simulation studies.

We extended the dependent-data TMLE framework first described in van der Laan [9] towards the estimation of a much larger class of parameters, such as the direct effect under interference. We have also shown that our framework can be extended to define interventions on the network itself. In particular, in Section 8.2 we described how one can estimate the post-intervention outcomes for interventions that statically or stochastically modify the initial network structure $F^{0}$. Furthermore, we no longer require complete independence of the baseline covariates for conducting valid statistical inference for our TMLE. Finally, we believe our work provides an important proof of concept, demonstrating that estimation and valid statistical inference for dependent data collected from a single network are possible in this large class of semi-parametric models.

We note that the TMLE update $\bar{Q}_{N}^{*}$ presented in this paper differs slightly from van der Laan [9] in terms of its suggested parametric submodel fluctuation, $\left\{\bar{Q}_{N}(\varepsilon): \varepsilon\right\}$, with the latter TMLE update being based on the following parametric submodel: Logit $\bar{Q}_{N}(\varepsilon)=\operatorname{Logit} \bar{Q}_{N}+\varepsilon \bar{g}_{0}^{*} / \bar{g}_{0}$. Both of these fluctuations result in TMLEs with equivalent asymptotic properties, as both updates solve the same empirical score equation. However, the two may differ in their finite sample properties. In particular, the TMLE we present here may be less sensitive to practical positivity violations, while providing similar bias reduction as the TMLE from van der Laan [9]. We also note that the TMLE update presented here may be less computationally intensive, since it only requires $N$ evaluations of the clever covariate $\left[\bar{g}_{N}^{*} / \bar{g}_{N}\right]\left(A_{i}^{s} \mid W_{i}^{s}\right)$, for $i=1, \ldots, N$. The TMLE algorithm proposed in van der Laan [9] may require computing $\bar{g}_{N}^{*} / \bar{g}_{N}\left(a_{i}^{s} \mid W_{i}^{s}\right)$ for every $a_{i}^{s}$ in the support of $A_{i}^{* s}$, for $i=1, \ldots, N$ (i.e., all $a_{i}^{s}$ such that $\left.g_{i}^{* S}\left(a_{i}^{S} \mid W_{i}^{S}\right)>0\right)$ due to its specific parametric submodel update.

We now note a few possible directions for future research. First, additional simulation studies should explore the performance of our TMLE in more complex networks, such as, networks generated from the preferential 
attachment model with power law node degree distribution [48] or networks generated under the small world model [49]. Second, it would be of interest to study how our proposed framework may be applied to estimate the change in the observed sample-average outcome when an intervention is applied to another community with a different network structure and different distribution of baseline covariates, a notion known as transportability [50-52]. Third, it is of scientific interest to explore how our estimation framework can be extended to real-world problems in which data on only a subsample of the full network is available. Moreover, the network structure on the observed units themselves is frequently not fully known, in which case it may be necessary to incorporate the uncertainty introduced by inferring the network structure from the observed data [53]. Finally, future work will investigate the estimation of causal parameters in longitudinal settings where the effect of a single time point intervention can propagate over time through the network, as is typically the case when one describes contagion in social networks [54, 55].

\section{Acknowledgments}

The authors would like to thank Elizabeth (Betsy) Ogburn for helpful discussions.

\section{Funding}

This research was supported by NIH grant R01 AI074345-07.

\section{A Simulation Study. Data generating distribution}

We implemented a simulation with observed data consisting of $N$ dependent units $O=(F, W, A, Y)$, where $F=\left(F_{1}, \ldots, F_{N}\right)$ is a vector of friends for each unit, $W=\left(W_{1}, \ldots, W_{N}\right)$ is a vector of baseline covariates, $A=$ $\left(A_{1}, \ldots, A_{N}\right)$ is a vector of binary treatments and $Y=\left(Y_{1}, \ldots, Y_{N}\right)$ is a vector of binary outcomes. The data for each unit $i=1, \ldots, N$ is generated as,

$$
\begin{array}{rc}
W_{i} & \sim \operatorname{Ber}(0.35) \\
\left|F_{i}\right| & \sim U(0,1,2) \\
F_{i}|| F_{i} \mid & \sim \operatorname{Sample}_{\left|F_{i}\right|}(\{1, \ldots, N\} \backslash i) \\
A_{i} \mid W, F_{i} & \sim \operatorname{Ber}\left(g_{0}\left(W_{i},\left(W_{j}: j \in F_{i}\right)\right)\right) \\
Y_{i} \mid A, W, F_{i} & \sim \operatorname{Ber}\left(\bar{Q}_{0}\left(A_{i}, W_{i},\left(A_{j}, W_{j}: j \in F_{i}\right)\right)\right),
\end{array}
$$

where $F_{i} \in\{1, \ldots, n\}$ is a set of of unit indices of size $\left|F_{i}\right|$ randomly sampled without replacement, $A_{i}$ is generated conditionally on the entire vector of baseline covariates $W$ and $Y_{i}$ is generated conditionally on $W$ and all treatment assignments $A$.

We generate $A$ with $g_{0}$ that depends on unit and unit's friends' baseline covariates,

$$
\bar{g}_{0}\left(W_{i},\left(W_{j}: j \in F_{i}\right)\right)=\operatorname{expit}\left(-1.2+1.5 W_{i}+\sum_{j \in F_{i}} 0.6 W_{j}\right),
$$

with $\bar{Q}_{0}$ defined as

$$
\bar{Q}_{0}\left(A_{i}, W_{i},\left(A_{j}, W_{j}: j \in F_{i}\right)\right)=\operatorname{expit}\left(-2.5+1.5 W_{i}+0.5 A_{i}+\sum_{j \in F_{i}} 1.5 W_{j}+\sum_{j \in F_{i}} 1.5 A_{j}\right)
$$

\section{B Consistency of the asymptotic variance estimator}

Theorem B.1. We make the following assumptions: 
Uniform convergence: Assume that as $N \rightarrow \infty$ we have $\sup _{a^{s}, w^{s}}\left|\bar{Q}_{N}\left(a^{s}, w^{s}\right)-\bar{Q}\left(a^{s}, w^{s}\right)\right| \stackrel{P}{\rightarrow} 0$, $\sup _{a^{s}, w^{s}}\left|\bar{g}_{N}^{*}\left(a^{s}, w^{s}\right)-\bar{g}^{*}\left(a^{s}, w^{s}\right)\right| \stackrel{P}{\rightarrow} 0, \sup _{a^{s}, w^{s}}\left|\bar{g}_{N}\left(a^{s}, w^{s}\right)-\bar{g}\left(a^{s}, w^{s}\right)\right| \stackrel{P}{\rightarrow} 0$ and $\sup _{w \in w} \mid \int_{a^{s}} \bar{Q}_{N}\left(a^{s}, w_{i}^{s}(w)\right) g_{i}^{*}\left(a^{s} \mid w\right)-$ $\int_{a^{s}} \bar{Q}\left(a^{s}, w^{s}(w)\right) g_{i}^{*}\left(a^{s} \mid w\right) \mid \stackrel{P}{\rightarrow} 0$, for each $i=1, \ldots, N$.

$L^{2}$-norm convergence of the i-specific target parameters: Assume that $d_{L^{2}}\left(\Psi_{i, N^{\prime}}^{*} \Psi_{i}^{*}\right) \rightarrow 0$, for each $i=$ $1, \ldots, N$;

Positivity: Assume with probability 1 that $\sup _{\left(a^{s}, w^{s}\right) \in\left(\mathcal{A}^{s}, w w^{s}\right)} \frac{\bar{g}^{*}}{\bar{g}}\left(a^{s}, w^{s}\right)<\infty$. Similarly, assume that $\sup _{\left(a^{s}, w^{s}\right) \in\left(\mathcal{A}^{s}, w^{s}\right)} \frac{\bar{g}_{N}^{*}}{\bar{g}_{N}}\left(a^{s}, w^{s}\right)<\infty$ with probability 1.

Boundedness of the asymptotic variance: Assume that the asymptotic variance of the TMLE $\sigma^{2}$ converges to $c<\infty$;

Boundedness of the outcomes: Assume that $\max _{i}\left|Y_{i}\right|<\infty$;

Universal bound on connectivity between units: Assume that $\sup \left|F_{i}\right|<K$, for all $i=1, \ldots, N$, where $K>\infty$ is a universal constant that doesn't depend on $N$; and

Symmetry of friend relationships: Assume that for any unit $i$, if another unit $j$ is in $i$ 's friend set $F_{i}$, i.e., $j \in F_{i}$, it must follow that $i \in F_{j}$.

Then the following plug-in estimator

$$
\sigma_{N}^{2}:=\frac{1}{N} \sum_{i=1}^{N} \sum_{j=1}^{N} R(i, j)\left[D_{N, i}(O) D_{N, j}(O)\right]
$$

is consistent for

$$
\sigma^{2}:=\lim _{N \rightarrow \infty} \frac{1}{N} \sum_{i=1}^{N} \sum_{j=1}^{N} R(i, j) E\left[D_{i}(O) D_{j}(O)\right]
$$

where

$$
\begin{gathered}
D_{N, i}(O)=\left[\frac{\bar{g}_{N}^{*}}{\bar{g}_{N}}\left(A_{i}^{s} \mid W_{i}^{s}\right)\left(Y_{i}-\bar{Q}_{N}\left(A_{i}^{s}, W_{i}^{s}\right)\right)\right]+\left[\int_{a^{s}} \bar{Q}_{N}\left(a^{s}, W_{i}^{s}\right) g_{i}^{*}\left(a^{s} \mid W\right)-\Psi_{i, N}^{*}\left(\bar{Q}_{N}, Q_{W, N}\right)\right], \\
D_{i}(O)=\left[\frac{\bar{g}^{*}}{\bar{g}}\left(A_{i}^{s} \mid W_{i}^{s}\right)\left(Y_{i}-\bar{Q}\left(A_{i}^{s}, W_{i}^{s}\right)\right)\right]+\left[\int_{a^{s}} \bar{Q}\left(a^{s}, W_{i}^{s}\right) g_{i}^{*}\left(a^{s} \mid W\right)-\Psi_{i}\left(\bar{Q}, Q_{W}\right)\right], \\
\Psi_{i}\left(\bar{Q}, Q_{W}\right)=\int_{w} \int_{a} \bar{Q}\left(a_{i}^{s}(a, w), w_{i}^{s}(w)\right) g^{*}(a: \mid: w) d Q_{W}(w), \\
\Psi_{N, i}\left(\bar{Q}_{N}^{*}, Q_{W, N}\right)=\int_{w} \int_{a} \bar{Q}_{N}^{*}\left(a_{i}^{s}(a, w), w_{i}^{s}(w)\right) g^{*}(a: \mid: w) d Q_{W, N}(w)
\end{gathered}
$$

and

$$
\int_{a^{s}} \bar{Q}_{N}\left(a^{s}, W_{i}^{s}\right) g_{i}^{*}\left(a^{s} \mid W\right)=\int_{a} \bar{Q}_{N}^{*}\left(a_{i}^{s}(a, W), w_{i}^{s}(W)\right) g^{*}(a: \mid: W) .
$$

Proof Part 1. Define

$$
\sigma^{2}(N):=\frac{1}{N} \sum_{i=1}^{N} \sum_{j=1}^{N} R(i, j)\left[D_{i}(O) D_{j}(O)\right],
$$

where $D_{i}:=D_{i}\left(\bar{g}^{*}, \bar{g}, \bar{Q}, \Psi_{i}^{*}\right)$. We first prove that $\sigma_{N}^{2}=\sigma^{2}(N)+o_{p}(1)$ or equivalently that 


$$
\begin{aligned}
\sigma_{N}^{2}-\sigma^{2}(N) & =\frac{1}{N} \sum_{i, j} R(i, j) D_{i, N} D_{j, N}-\frac{1}{N} \sum_{i, j} R(i, j) D_{i} D_{j} \\
& =\frac{1}{N} \sum_{i, j} R(i, j)\left[\left(D_{i, N}-D_{i}\right) D_{j}+D_{i, N}\left(D_{j, N}-D_{j}\right)\right] \\
& =o_{P}(1) .
\end{aligned}
$$

The above expression implies that our claim would follow if we were to show that

$$
\begin{aligned}
\frac{1}{N} \sum_{i, j} R(i, j) D_{i, N}\left(D_{j, N}-D_{j}\right) & =o_{P}(1) \quad \text { and } \\
\frac{1}{N} \sum_{i, j} R(i, j)\left(D_{i, N}-D_{i}\right) D_{j} & =o_{P}(1) .
\end{aligned}
$$

We already assumed that $\max _{i}\left|D_{i, N}\right|<M$ with probability 1 , for some constant $M>\infty$. Thus we have:

$$
\begin{aligned}
\frac{1}{N} \sum_{i, j} R(i, j) D_{i, N}\left(D_{j, N}-D_{j}\right) & \leq \frac{1}{N} \sum_{i, j} R(i, j) \max _{i}\left|D_{i, N}\right| \max _{j}\left|D_{j, N}-D_{j}\right| \\
& =\frac{1}{N} \sum_{i=1}^{N} \max _{i}\left|D_{i, N}\right| \sum_{j=1}^{N} R(i, j) \max _{j}\left|D_{j, N}-D_{j}\right| \\
& =\max _{i \in\{1, \ldots, N\}}\left|D_{i, N}\right| \sum_{j=1}^{N} R(i, j) \max _{j \in\{1, \ldots, N\}}\left|D_{j, N}-D_{j}\right| \\
& \leq K^{2} M \max _{j \in\{1, \ldots, N\}}\left|D_{j, N}-D_{j}\right| .
\end{aligned}
$$

Define $e_{N}:=\max _{j}\left|D_{j, N}-D_{j}\right|$. The sum above is bounded by $e_{N} K^{2} M_{2}$ and showing that $e_{N} \stackrel{P}{\rightarrow} 0$ would prove that the first and second sums both go to zero with probability 1 . Note that

$$
\max _{j \in\{1, \ldots, N\}}\left|D_{j, N}(O)-D_{j}(O)\right| \leq \max _{j \in\{1, \ldots, N\}} \sup _{o \in O}\left|D_{j, N}(o)-D_{j}(o)\right|,
$$

Thus showing that $\sup _{o \in O}\left|D_{j, N}(o)-D_{j}(o)\right| \stackrel{P}{\rightarrow} 0$, for any $j=1, \ldots, N$, would prove our claim. This implies that we need to show that

$$
\sup _{\left(y, a^{s}, w^{s}\right) \in\left(y, A^{s}, w^{s}\right)}\left|\frac{\bar{g}_{N}^{*}}{\bar{g}_{N}}\left(a^{s}, w^{s}\right)\left(y-\bar{Q}_{N}\left(a^{s}, w^{s}\right)\right)-\frac{\bar{g}^{*}}{\bar{g}}\left(a^{s}, w^{s}\right)\left(y-\bar{Q}\left(a^{s}, w^{s}\right)\right)\right| \stackrel{P}{\rightarrow} 0,
$$

and

$$
\sup _{w \in W}\left|\left(\int_{a^{s}} \bar{Q}_{N}\left(a^{s}, w^{s}(w)\right) g_{i}^{*}\left(a^{s} \mid w\right)-\Psi_{N, i}^{*}\right)-\left(\int_{a^{s}} \bar{Q}\left(a^{s}, w^{s}(w)\right) g_{i}^{*}\left(a^{s} \mid w\right)-\Psi_{i}^{*}\right)\right| \stackrel{P}{\rightarrow} 0,
$$

where $\left(y, \mathcal{A}^{s}, W^{s}\right)$ is the common support of $\left(Y_{i}, A_{i}^{s}, W_{i}^{s}\right)$, across all $i$. Note that

$$
\begin{aligned}
& \sup _{\left(y, a^{s}, w^{s}\right)}\left|\frac{y \bar{g}_{N}^{*}\left(a^{s}, w^{s}\right)}{\bar{g}_{N}\left(a^{s}, w^{s}\right)}-\frac{y \bar{g}^{*}\left(a^{s}, w^{s}\right)}{\bar{g}\left(a^{s}, w^{s}\right)}-\frac{\bar{g}_{N}^{*}\left(a^{s}, w^{s}\right)}{\bar{g}_{N}\left(a^{s}, w^{s}\right)} \bar{Q}_{N}\left(a^{s}, w^{s}\right)+\frac{\bar{g}^{*}\left(a^{s}, w^{s}\right)}{\bar{g}\left(a^{s}, w^{s}\right)} \bar{Q}\left(a^{s}, w^{s}\right)\right| \\
\leq & \sup _{y \in \mathcal{Y}}|Y| \sup _{\left(a^{s}, w^{s}\right)}\left|\frac{\bar{g}_{N}^{*}\left(a^{s}, w^{s}\right)}{\bar{g}_{N}\left(a^{s}, w^{s}\right)}-\frac{\bar{g}^{*}\left(a^{s}, w^{s}\right)}{\bar{g}\left(a^{s}, w^{s}\right)}\right|+\sup _{\left(a^{s}, w^{s}\right)}\left|\frac{\bar{g}_{N}^{*}\left(a^{s}, w^{s}\right)}{\bar{g}_{N}\left(a^{s}, w^{s}\right)} \bar{Q}_{N}\left(a^{s}, w^{s}\right)-\frac{\bar{g}^{*}\left(a^{s}, w^{s}\right)}{\bar{g}\left(a^{s}, w^{s}\right)} \bar{Q}\left(a^{s}, w^{s}\right)\right| .
\end{aligned}
$$

By assumption, $\sup _{y \in y}|Y|=c_{Y}>\infty$. It now follows that

$$
\begin{aligned}
& c_{Y} \sup _{\left(a^{s}, w^{s}\right)}\left|\frac{\bar{g}_{N}^{*}\left(a^{s}, w^{s}\right)}{\bar{g}_{N}\left(a^{s}, w^{s}\right)}-\frac{\bar{g}^{*}\left(a^{s}, w^{s}\right)}{\bar{g}\left(a^{s}, w^{s}\right)}\right| \\
= & c_{Y} \sup _{\left(a^{s}, w^{s}\right)}\left|\left(\bar{g}_{N}^{*}\left(a^{s}, w^{s}\right)-\bar{g}^{*}\left(a^{s}, w^{s}\right)\right) \frac{1}{\bar{g}\left(a^{s}, w^{s}\right)}+\bar{g}_{N}^{*}\left(a^{s}, w^{s}\right)\left(\frac{1}{\bar{g}_{N}\left(a^{s}, w^{s}\right)}-\frac{1}{\bar{g}\left(a^{s}, w^{s}\right)}\right)\right| \stackrel{P}{\rightarrow} 0,
\end{aligned}
$$


where we used that $\sup _{\left(a^{s}, w^{s}\right)}\left(1 / \bar{g}\left(a^{s}, w^{s}\right)\right)>\infty$ and $\sup _{\left(a^{s}, w^{s}\right)}\left(1 / \bar{g}_{N}\left(a^{s}, w^{s}\right)\right)>\infty$ along with the uniform convergence of $\bar{g}_{N}$, and $\bar{g}_{N}^{*}$. Similarly, it follows for the second term that

$$
\begin{aligned}
& \sup _{\left(a^{s}, w^{s}\right)}\left|\left(\frac{\bar{g}_{N}^{*}\left(a^{s}, w^{s}\right)}{\bar{g}_{N}\left(a^{s}, w^{s}\right)}-\frac{\bar{g}^{*}\left(a^{s}, w^{s}\right)}{\bar{g}\left(a^{s}, w^{s}\right)}\right) \bar{Q}\left(a^{s}, w^{s}\right)+\frac{\bar{g}_{N}^{*}\left(a^{s}, w^{s}\right)}{\bar{g}_{N}\left(a^{s}, w^{s}\right)}\left(\bar{Q}_{N}\left(a^{s}, w^{s}\right)-\bar{Q}\left(a^{s}, w^{s}\right)\right)\right| \\
\leq & \sup _{\left(a^{s}, w^{s}\right)}\left|\bar{Q}_{0}\left(a^{s}, w^{s}\right)\right|\left|\frac{\bar{g}_{N}^{*}\left(a^{s}, w^{s}\right)}{\bar{g}_{N}\left(a^{s}, w^{s}\right)}-\frac{\bar{g}^{*}\left(a^{s}, w^{s}\right)}{\bar{g}\left(a^{s}, w^{s}\right)}\right|+\sup \frac{\bar{g}_{N}^{*}\left(a^{s}, w^{s}\right)}{\bar{g}_{N}\left(a^{s}, w^{s}\right)}\left|\bar{Q}_{N}\left(a^{s}, w^{s}\right)-\bar{Q}\left(a^{s}, w^{s}\right)\right| \stackrel{P}{\rightarrow} 0,
\end{aligned}
$$

where we used the uniform convergence of $\bar{Q}_{N}, \bar{g}_{N}$, and $\bar{g}_{N}^{*}$. We also note that

$$
\sup _{w \in W}\left|\left(\int_{a^{s}} \bar{Q}_{N}\left(a^{s}, w^{s}(w)\right) g_{i}^{*}\left(a^{s} \mid w\right)-\Psi_{N, i}^{*}\right)-\left(\int_{a^{s}} \bar{Q}\left(a^{s}, w^{s}(w)\right) g_{i}^{*}\left(a^{s} \mid w\right)-\Psi_{i}^{*}\right)\right| \stackrel{P}{\rightarrow} 0
$$

directly follows, which finally proves that $e_{N}=\max _{j \in\{1, \ldots, N\}}\left|D_{j, N}-D_{j}\right| \stackrel{P}{\rightarrow} 0$ and hence that

$$
\sigma_{N}^{2}=\sigma^{2}(N)+o_{p}(1)
$$

Part 2. To show consistency of $\sigma^{2}(N)$ we show that: (1) $\left.E\left(\sigma^{2}(N)\right) \rightarrow \sigma^{2}\right)$ and (2) $\operatorname{Var}\left(\sigma^{2}(N)\right) \rightarrow 0$, followed by the application of Chebyshev's inequality. Note that (1) follows trivially from the definition of $\sigma^{2}$. To show that the variance of the above plug-in estimator $\sigma_{N}^{2}$ goes to zero we use a similar argument to the one employed in the second half of the proof of Proposition 1 in [56]. Consider the variance of $\sigma^{2}(N)$ :

$$
\begin{aligned}
\operatorname{Var}\left(\sigma^{2}(N)\right) & =\frac{1}{N^{2}} \operatorname{Var}\left(\sum_{i, j} R(i, j)\left[D_{i}(O) D_{j}(O)\right]\right) \\
& =\frac{1}{N^{2}}\left(\sum_{i, j, k, l} \operatorname{Cov}\left(R(i, j)\left[D_{i}(O) D_{j}(O)\right], R(k, l)\left[D_{k}(O) D_{l}(O)\right]\right)\right) \\
& =\frac{1}{N^{2}}\left(\sum_{i, j, k, l} R(i, j) R(k, l) \operatorname{Cov}\left(\left[D_{i}(O) D_{j}(O)\right],\left[D_{k}(O) D_{l}(O)\right]\right)\right)
\end{aligned}
$$

Note that the terms in the above sum are nonzero only when $R(i, j)=R(k, l)=1$. Now consider now the individual terms of this sum:

$$
\begin{aligned}
\xi_{i j k l} & =\operatorname{Cov}\left(\left[D_{i}(O) D_{j}(O)\right],\left[D_{k}(O) D_{l}(O)\right]\right) \\
& =E\left(\left[D_{i}(O) D_{j}(O)\right]\left[D_{k}(O) D_{l}(O)\right]\right)-E\left(D_{i}(O) D_{j}(O)\right) E\left(D_{k}(O) D_{l}(O)\right) .
\end{aligned}
$$

Note that $E\left[D_{i}(O)\right]=0$, for any $i=1, \ldots, N$. Our task is to show that the number of nonzero terms $\xi_{i j k l}$ in the sum over $(i, j, k . l)$ is $O(N)$, where $\xi_{i j k l} \neq 0$ only when either of the following four conditions holds: $(1) R(i, k)=1$ or (2) $R(j, k)=1$ or $(3) R(i, l)=1$ or $(4) R(j, l)=1$. Note that we already assumed that the maximum number of friends $\left(\max _{i=1, \ldots, N}\left|F_{i}\right|\right)$ is bounded by some universal constant $K>\infty$, where $K$ does not depend on $N$.

We proceed to sum over indices $(i, j, k, l)$, counting the possible number of nonzero terms in each sum. For a sum over $i$ there are $O(N)$ nonzero terms. For each fixed $i$, the sum over $j=1, \ldots, N$ can have $O\left(K^{2}\right)$ nonzero terms since its constrained by condition $R(i, j)=1$. Note that for each fixed $i$ there are at most $K^{2}$ terms in $j$ that may satisfy $R(i, j)=1$. Next, fix $(i, j)$ and sum over $(k, l)$. Note that these summands will be nonzero only if conditions (1) or (2) or (3) or (4) are satisfied and if $R(k, l)=1$. First, consider the case when $R(i, k)=1$ or $R(j, k)=1$. The sum over $k$ then must net $O\left(2 K^{2}\right)$ nonzero elements. However, the sum over $l$ is still under the constraint $R(k, l)=1$, implying that the sum over $k$ and $l$ is $O\left(2 K^{4}\right)$, with total sum over $(i, j, k, l)$ being $O\left(2 K^{6} N\right)$. One proceeds similarly for cases with $R(i, l)=1$ or $R(j, l)=1$, summing first over $l$ for fixed $(i, j)$ and then over $k$, also resulting in $O\left(2 K^{6} N\right)$ terms. Thus, the total number of nonzero terms is $O\left(4 K^{6} N\right)$ and the claim follows.

\section{Notation index}

$O=(W, A, Y):$ the observed data on $N$ units 
$W=\left(W_{1}, \ldots, W_{N}\right)$ : the observed baseline covariates on $N$ dependent units

$F=\left(F_{1}, \ldots, F_{N}\right)$ : the observed network on $N$ units ("network profile")

$A=\left(A_{1}, \ldots, A_{N}\right)$ : the observed exposures on $N$ dependent units

$A^{*}=\left(A_{1}^{*}, \ldots, A^{*}\right)$ : intervened exposures sampled under conditional distribution $G^{*}$, given $W$

$Y=\left(Y_{1}, \ldots, Y_{N}\right)$ : the observed outcomes on $N$ connected units

$W_{i}^{s}=w_{i}^{s}(W), A_{i}^{s}=a_{i}^{s}(A, W)$ : fixed-dimension summary measures, dimensionality is the same across alli.

$O^{s}=\left(W^{s}, A^{s}, Y\right)$ : observed summary data, where $W^{s}=\left(W_{1}^{s}, \ldots, W_{N}^{s}\right), A^{s}=\left(A_{1}^{s}, \ldots, A_{N}^{s}\right)$, for $i=1, \ldots, N$

$O^{* s}=\left(W^{s}, A^{* s}, Y^{*}\right)$ : summary data sampled from post-intervention distribution under stochastic interventiong ${ }^{*}$, with $A^{* s}=\left(A_{1}^{* s}, \ldots, A_{N}^{* s}\right)$ and $Y^{*}=\left(Y_{1}^{*}, \ldots, Y_{N}^{*}\right)$

$P_{0}^{N}$ : true joint distribution of the observed data $O$

$p_{0}^{N}$ : true joint density of the observed data $O$

$Q_{W, 0}$ : true joint distribution of $N$ observed baseline covariates $W$

$q_{W, 0}:$ true joint density of $N$ observed baseline covariates $W$

$G_{0}(A: \mid: W)$ : joint conditional distribution for the observed exposures $A$, given baseline covariates $W$

$g_{0}(A: \mid: W)$ : joint conditional density for the observed exposures $A$, given baseline covariates $W$

$G_{0}\left(A_{i} \mid W_{i}^{s}\right)$ : common-in- $i$ conditional distribution for the observed exposure $A_{i}$, given the $i$-specific summary measure of the baseline covariates

$g_{0}\left(A_{i} \mid W_{i}^{s}\right)$ : common-in- $i$ conditional density for the observed exposure $A_{i}$, given the $i$-specific summary measure of the baseline covariates

$G^{*}\left(A^{*}: \mid: W\right)$ : user-specified distribution of the intervened network exposure vector $A^{*}=\left(A_{1}^{*}, \ldots, A_{N}^{*}\right)$, conditional on baseline covariates $W=\left(W_{1}, \ldots, W_{N}\right)$

$g^{*}\left(A^{*}: \mid: W\right)$ : density for the user-specified stochastic intervention of the intervened exposure vector $A^{*}=\left(A_{1}^{*}, \ldots, A_{N}^{*}\right)$, conditional on all baseline covariates $W=\left(W_{1}, \ldots, W_{N}\right)$

$\bar{Q}_{0}\left(a^{s}, w^{s}\right)$ : conditional expectation of the outcome, defined as $E_{P_{0}^{N}}\left[Y_{i} \mid A_{i}^{s}=a^{s}, W_{i}^{s}=w^{s}\right]$, assumed common across $i$ when conditioned on the same fixed summary measure values $a^{s}, w^{s}$

$\bar{H}_{i, 0}\left(A_{i}^{s}, W_{i}^{s}\right): i$-specific summary measure distribution for $\left(A_{i}^{s}, W_{i}^{s}\right)$

$h_{i, 0}\left(A_{i}^{s}, W_{i}^{s}\right): i$-specific density for the distribution $\bar{H}_{i, 0}\left(A_{i}^{s}, W_{i}^{s}\right)$

$H_{i, 0}^{*}\left(A_{i}^{s}, W_{i}^{s}\right): i$-specific summary measure distribution for $\left(A_{i}^{* s}, W_{i}^{s}\right)$

$h_{i, 0}^{*}\left(A_{i}^{* s}, W_{i}^{s}\right): i$-specific density for the distribution $\bar{H}_{i, 0}^{*}\left(A_{i}^{* s}, W_{i}^{S}\right)$

$\bar{h}_{0}\left(\bar{A}^{s}, \bar{W}^{s}\right)$ : mixture density $1 / N \sum_{i} h_{i, 0}$ determined by $g_{0}$ and $Q_{W, 0}$, with $\left(\bar{A}^{s}, \bar{W}^{s}\right)$ being a random variable sampled from $\bar{h}_{0}$

$\bar{h}_{0}^{*}\left(\bar{A}^{* s}, \bar{W}^{s}\right)$ : mixture density $1 / N \sum_{i} h_{i, 0}^{*}$ determined by $g^{*}$ and $Q_{W, 0}$, with $\left(\bar{A}^{* s}, \bar{W}^{s}\right)$ being a random variable sampled from $\bar{h}_{0}^{*}$

$\bar{g}_{0}\left(\bar{A}^{s}: \mid:\right.$ barW $\left.W^{s}\right)$ : the conditional mixture density implied by factorization $\bar{h}_{0}\left(\bar{A}^{s}, \bar{W}^{s}\right)=\bar{g}_{0}\left(\bar{A}^{s}: \mid\right.$ : $\left.\operatorname{bar} W^{s}\right) \bar{Q}_{W^{s, 0}}\left(\bar{W}^{s}\right)$

$\bar{g}_{0}^{*}\left(\bar{A}^{* s}: \mid:\right.$ barW $\left.W^{s}\right)$ : the conditional mixture density implied by factorization $\bar{h}^{*}\left(\bar{A}^{* s}, \bar{W}^{s}\right)=\bar{g}_{0}^{*}\left(\bar{A}^{* s}: \mid\right.$ : $\left.\operatorname{bar} W^{s}\right) \bar{Q}_{W^{s}, 0}\left(\bar{W}^{s}\right)$.

\section{Notes}

${ }^{1}$ This assumption will be also referred to as the weak dependence of $\mathbf{W}$.

\section{References}

[1] Neyman ]. Sur les applications de la theorie des probabilites aux experiences agricoles: Essai des principes (In Polish). English translation by DM Dabrowska and TP Speed (1990). Stat Sci. 1923:5:465-480.

[2] Hudgens MG, Halloran ME. Toward causal inference with interference. ] Am Stat Assoc. 2008;103.

[3] Sobel ME. What do randomized studies of housing mobility demonstrate?. ] Am Stat Assoc. 2006;101:1398-1407.

[4] Basse CW, Airoldi EM. Optimal design of experiments in the presence of network-correlated outcomes. ArXiv e-prints 2015.

[5] Christakis NA, Fowler ]H. The spread of obesity in a large social network over 32 years. N Engl ] Med. 2007;357:370-379.

[6] Christakis NA, Fowler ]H. Social contagion theory: examining dynamic social networks and human behavior. Stat Med. 2013;32:556-577.

[7] van der Laan M], Rose S. Targeted learning: causal inference for observational and experimental data Springer Series in Statistics Springer-Verlag, 2011.

[8] van der Laan M], Rubin D. Targeted maximum likelihood learning. Int] Biostat. 2006;2.

[9] van der Laan M]. Causal inference for a population of causally connected units. ] Causal Inference. 2014;2:1-62. 
[10] Tchetgen E], vanderWeele T]. On causal inference in the presence of interference. Stat Methods Med Res. 2012;21:55-75.

[11] Ogburn EL, vanderWeele T]. Vaccines contagion, and social networks. ArXiv e-prints 2014.

[12] VanderWeele T], An W. Social networks and causal inference. In Handbook of causal analysis for social research Springer, 2013:353-374.

[13] VanderWeele T], Tchetgen E], Halloran ME. Interference and sensitivity analysis. Stat Sci. 2014;29:687-706.

[14] Aral S, Walker D. Identifying social influence in networks using randomized experiments. IEEE Intell Syst. 2011;26:91-96.

[15] Aral S, Walker D. Tie strength, embeddedness, and social influence: a large-scale networked experiment. Manage Sci. 2014;60:1352-1370.

[16] Aronow PM, Samii C. Estimating average causal effects under interference between units. ArXiv e-prints 2013.

[17] Bowers ], Fredrickson MM, Panagopoulos C. Reasoning about interference between units: A general framework. Polit Anal. 2013;21:97-124.

[18] Choi DS. Estimation of monotone treatment effects in network experiments ArXiv e-prints 2014.

[19] Liu L, Hudgens MG. Large sample randomization inference of causal effects in the presence of interference. ] Am Stat Assoc. 2014;109:288-301.

[20] Rosenbaum PR. Interference between units in randomized experiments. ] Am Stat Assoc. 2007;102:191-200.

[21] Toulis P, Kao E. Estimation of causal peer influence effects. Proceedings of The 30th International Conference on Machine Learning 2013:1489-1497.

[22] Walker D, Muchnik L. Design of randomized experiments in networks. Proc IEEE. 2014;102:1940-1951.

[23] Lyons R. The spread of evidence-poor medicine via flawed social-network analysis. Stat Polit Policy. 2010;2:1-26.

[24] VanderWeele T]. Sensitivity analysis for contagion effects in social networks. Sociol Method Res. 2011;40:240-255.

[25] VanderWeele T]. Inference for influence over multiple degrees of separation on a social network. Stat Med. 2013;32:591-596.

[26] VanderWeele T], Ogburn EL, Tchetgen Tchetgen E]. Why and when "flawed" social network analyses still yield valid tests of no contagion. Stat Polit Policy. 20123;3.

[27] Steglich C, Snijders TA, Pearson M. Dynamic networks and behavior: Separating selection from influence. Sociological Method. 2010;40:329-393.

[28] Balzer L, Petersen M, van der Laan M. Targeted estimation and inference for the sample average treatment effect. U.C. Berkeley Division of Biostatistics Working Paper Series 2015.

[29] van der Laan M], Hubbard AE, Pajouh SK. Statistical inference for data adaptive target parameters. U.C. Berkeley Division of Biostatistics Working Paper Series 2013.

[30] Robins ]M. Addendum to "a new approach to causal inference in mortality studies with a sustained exposure period-application to control of the healthy worker survivor effect. Computers \& Mathematics with Applications. 1987;14:923-945.

[31] Sofrygin O. van der Laan M]. Targeted maximum likelihood estimation for network data. R package version. 2015;0.1.0.

[32] Cill RD, Robins ]M. Causal inference for complex longitudinal data: the continuous case. Ann Stat 2001:1785-1811.

[33] Robins ]M. A graphical approach to the identification and estimation of causal parameters in mortality studies with sustained exposure periods. J Chronic Diseases. 1987;40(Suppl 2):139S-161S.

[34] Robins ]M. Causal inference from complex longitudinal data. In Latent variable modeling and applications to causality Springer, 1997:69-117.

[35] Robins ]M. [Choice as an alternative to control in observational studies]: comment. Stat Sci 1999;281-293.

[36] Yu Z, van der Laan M]. Measuring treatment effects using semiparametric models. U.C. Berkeley Division of Biostatistics Working Paper Series 2003.

[37] Dawid AP, Didelez V Identifying the consequences of dynamic treatment strategies: a decision-theoretic overview. Stat Surveys. 2010;4:184-231.

[38] Muñoz ID. van der Laan M. Population intervention causal effects based on stochastic interventions. Biometrics. 2012;68:541-549.

[39] Robins ]M, Richardson T. Alternative graphical causal models and the identification of direct effects. Causality and Psychopathology: Finding the Determinants of Disorders and Their Cures 2010:103-158.

[40] Zheng W, van der Laan M]. Causal mediation in a survival setting with time-dependent mediators. U.C. Berkeley Division of Biostatistics Working Paper Series 2012.

[41] Bickel P]. Efficient and adaptive estimation for semiparametric models. New York: Springer-Verlag, 1993.

[42] van der Vaart AW, Statistics Asymptotic. Cambridge Series in Statistical and Probabilistic Mathematics. Cambridge: Cambridge University Press, 1998.

[43] Sofrygin O, van der Laan M]. Semi-parametric estimation and inference for the mean outcome of the single time-point intervention in a causally connected population. U.C. Berkeley Division of Biostatistics Working Paper Series 2015.

[44] Gruber S, van der Laan M]. Targeted maximum likelihood estimation: A gentle introduction. U.C. Berkeley Division of Biostatistics Working Paper Series 2009.

[45] Core Team R. R: a language and environment for statistical computing R Foundation for Statistical Computing, 2015 Vienna.

[46] Miguel E, Worms Kremer M. identifying impacts on education and health in the presence of treatment externalities. Econometrica. 2004;72:159-217.

[47] Davey C, Aiken AM, Hayes R], Hargreaves ]R. Re-analysis of health and educational impacts of a school-based deworming programme in western Kenya: a statistical replication of a cluster quasi-randomized stepped-wedge trial. Int] Epidemiol 2015.

[48] Barabási AL, Albert R. Emergence of scaling in random networks. Science. 1999;286:509-512.

[49] Watts D], Strogatz SH. Collective dynamics of 'small-world' networks. Nature. 1998;393:440-442.

[50] Bareinboim E, Pearl ]. A general algorithm for deciding transportability of experimental results. ] Causal Inference. 2013;1:107-134.

[51] Pearl ], Bareinboim E. Transportability of causal and statistical relations: a formal approach. Proceedings of the 25th National Conference on Artificial Intelligence (AAAl) 2011:247-254.

[52] Pearl ], Bareinboim E. External validity: from do-calculus to transportability across populations. Stat Sci. 2014;29:579-595.

[53] Goyal R, De Gruttola V, Blitzstein ]. Sampling networks from their posterior predictive distribution. Network Sci. 2014;2:107-131.

[54] Eckles D, Karrer B, Ugander ]. Design and analysis of experiments in networks: Reducing bias from interference. arXiv preprint 2014. 
[55] Ugander ], Karrer B, Backstrom L, Kleinberg ]. Graph cluster randomization: Network exposure to multiple universes. Proceedings of the 19th ACM SICKDD international conference on Knowledge discovery and data mining 2013:329-337.

[56] Aronow PM, Crawford FW, Zubizarreta ]R. Confidence intervals for means under constrained dependence arXiv preprint arXiv:1602.00359 2016. 